\title{
Molecular Information Technology
}

\author{
Klaus-Peter Zauner \\ School of Electronics and Computer Science \\ University of Southampton \\ Highfield, Southampton, SO17 1BJ \\ United Kingdom \\ kpz@ecs.soton.ac.uk \\ www.ecs.soton.ac.uk/ ${ }^{\sim} \mathrm{kpz} /$
}

Manuscript of material that appeared in:

Critical Reviews in Solid State and Material Sciences, Volume 30, Number 1, pp. 33-69, 2005.

The article is available from Taylor \& Francis at http: //taylorandfrancis.metapress . com/link. asp?id=w375573488537458

The text of this manuscript is close to the published article, but may not be identical. 


\section{ABSTRACT:}

Molecular materials are endowed with unique properties of unrivaled potential for high density integration of computing systems. Present applications of molecules range from organic semiconductor materials for low-cost circuits to genetically modified proteins for commercial imaging equipment. To fully realize the potential of molecules in computation, information processing concepts that relinquish narrow prescriptive control over elementary structures and functions are needed, and self-organizing architectures have to be developed. Investigations into qualitatively new concepts of information processing are underway in the areas of reaction-diffusion computing, self-assembly computing, and conformation-based computing. Molecular computing is best considered not as competitor for conventional computing, but as an opportunity for new applications. Microrobotics and bioimmersive computing are among the domains likely to benefit from advances in molecular computing. Progress will depend on both novel computing concepts and innovations in materials. This article reviews current directions in the use of bulk and single molecules for information processing.

\section{KEY WORDS:}

polymer electronics, molecular switches, self-assembly computing, conformation-based computation, self-organizing materials, bioimmersive computing

\section{ACRONYMS:}

AC-Alternating current

ATP-Adenosine triphosphate

BR-Bacteriorhodopsin

BZ-Belousov-Zhabotinsky (reaction)

CMOS - Complementary metal oxide semiconductor

CPU—Central processing unit

DNA-Deoxyribonucleic acid

DRZ-Deoxyribozyme

FET - Field effect transistor

FRET-Fluorescence resonance energy transfer

LB - Langmuir-Blodgett (film)

LED — Light emitting diode

$\mathrm{MDH}$ - Malate dehydrogenase

NAND - Negation of logic AND operation

NMR - Nuclear magnetic resonance

NOR - Negation of logic OR operation

$\mathrm{NP}$ - Nondeterministic polynomial

PCR - Polymerase chain reaction

PET-Photoinduced electron transfer

RAM-Random access memory

RFID - Radio frequency identification

RNA - Ribonucleic acid

TTF-Tetrathiafulvalene

UV-Ultra violet

XOR-Exclusive OR logic operation 


\section{TABLE OF CONTENTS}

1 Introduction 3

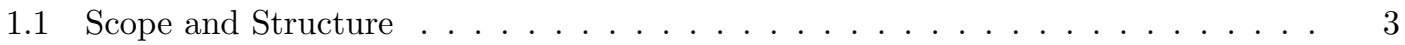

1.2 Organic Devices . . . . . . . . . . . . . . . . . . . . . . 4

2 Computing Concepts 5

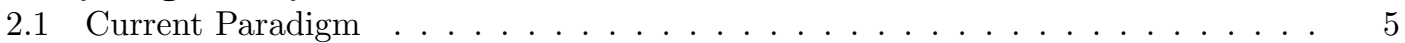

2.2 Unconventional Computing . . . . . . . . . . . . . . . . 8

3 Implementations 10

3.1 Carbon-based Semiconductors . . . . . . . . . . . . . . . . . . . . . . 10

3.1 .1 Polymer Electronics _. . . . . . . . . . . . . . . . . . . . 10

3.1 .2 Carbon Nanotubes . . . . . . . . . . . . . . . . . . . . . 12

3.2 Molecular Switches . . . . . . . . . . . . . . . . . . . . . . . . . 14

3.3 Bacteriorhodopsin: a Photonic Automaton . . . . . . . . . . . . . . . . 17

3.4 Excitable Chemical Media . . . . . . . . . . . . . . . . . . . . . . . . . . . 21

3.5 Self-assembly Computing . . . . . . . . . . . . . . . . . . . . 24

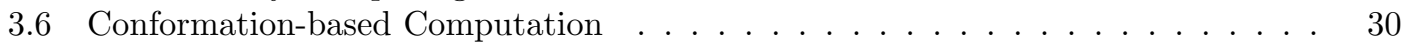

3.7 Allosterically Regulated Deoxyribozymes . . . . . . . . . . . . . . . . . . . 35

3.8 NMR Quantum Computing . . . . . . . . . . . . . . . . . . . . 36

4 Interfaces, Interconnections, and Architectures 37

4.1 Diffusion and Transport . . . . . . . . . . . . . . . . . . . 38

4.2 Uncontrolled Structures $\ldots \ldots \ldots \ldots$. . . . . . . . . . . . . . . . . 38

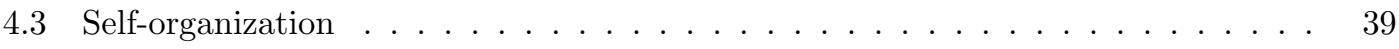

5 Perspectives 40

\section{INTRODUCTION}

\subsection{Scope and Structure}

A confluence of three research directions brings molecular information technology into focus. The feature size of semiconductor devices $[1,2]$ is approaching the scale of large macromolecules $[3,4]$. The enviable computational capabilities of organisms are increasingly traced to molecular mechanisms $[5,6]$. And, techniques to engineer molecular control structures directly into living cells start to emerge $[7,8]$. Current attempts to employ molecules for computation fall into two categories. Firstly, investigations aimed at obtaining molecules that mimic components of conventional computing devices. Transistors from carbon-based semiconductors and molecular logic gates are examples falling into this category. Secondly, explorations into innovative computation schemes to find information processing concepts suitable for exploiting the special characteristics of molecules. Computation based on reaction-diffusion systems and on self-assembly of macromolecules are in the latter category. The scope of the present review will encompass both of these areas. Nonetheless some fields that are part of molecular information technology have been excluded. Molecules have already found wide spread application in current computing devices in two areas, liquid crystals displays and organic dyes in recordable compact disks (CD-R). Both are not covered in the present review. The prospect of molecular computing attracted interest from the theoretical computer science community following the publication of Adleman's experimental work on DNA computing [9] and a considerable body of literature appeared. In a significant part of this, however, abstractions and models have been used that are ill suited to realization. The interested reader will find pointers to the literature in [10]; the present review will focus on realizable computing concepts.

The next section (2) provides a brief introduction to the basic concepts of present computing technology and then describes some unconventional computing concepts. The latter are of interest because the common paradigm of digital solid state circuits may not be the most suitable approach to utilizing molecules. Following the overview of computing schemes the current directions in implementing molecular computing are considered in section 3. The various molecular computing methods have been allocated to major categories. This is not unambiguously 
possible in every case. Ribozyme based logic gates, for example, combine aspects of switching, self-assembly and conformational computation all in one single system. Of particular importance for the practical realization of molecular computing devices is the infrastructure to interconnect molecular components into architectures and, in many cases, to interface these molecular architectures with existing information technology; section 4 is dedicated to this aspect. Finally an overview over the challenges and possibilities of molecular computing is offered in the concluding section. In the remaining part of this section the properties of molecules key to their application and potential in information technology will be described. The important differences to solid-state technology will also be outlined.

\subsection{Organic Devices}

The extraordinary diversity of the biological world has its roots in an enormous number of macromolecular structures that implement a wide variety of highly specific functions. These heterogeneous structures are assembled from a small set of molecular building blocks. The building blocks themselves are composed form a limited subset of the elements (mainly H, C, N, $\mathrm{O}, \mathrm{P}, \mathrm{S})$. Proteins provide a prominent example. In nature these are assembled from 20 types of amino acids (Gesteland [11] lists rare exceptions), which are themselves built up from between 10 and 27 atoms. The amino acids differ in size and shape as well as in their physical and chemical properties. Proteins are linear chains of amino acids linked by covalent chemical bonds. The chains are typically a few hundred amino acids long and their molecular mass corresponds to the equivalent of 10000 to several million hydrogen atoms [12]. There is no restriction on the order in which the different types of amino acids can occur in the chain and consequently the variety of chains is practically inexhaustible. For proteins only 60 amino acids long and formed from a set of 20 amino acids the number of distinct possibilities $\left(20^{60}\right)$ is about as large as the number of atoms in the known universe. A self-organizing folding process maps the combinatorial diversity of possible amino acid sequences into a vast variety of specific functional properties. Proteins thus grant substantial freedom to select specific stimuli to respond to, and to associate these with a response in an essentially arbitrary way.

Macromolecules have a size that situates them at the interface between micro and macro physics (table 1). They are small enough to exhibit quantum level features and to couple to

\begin{tabular}{r|c|r}
\multicolumn{3}{c}{ Table 1: Scale of molecular structures } \\
\hline (Supra-)Molecular structure & Size $[\mathrm{nm}]$ & Ref. \\
\hline Single wall carbon nano tube & $0.75-13$ & {$[13,14]$} \\
Cytochrome c & $2.6 \times 3.2 \times 3.3$ & {$[4]$} \\
Lysozyme & $1.9 \times 2.5 \times 4.3$ & {$[4]$} \\
Myoglobin & $2.1 \times 3.5 \times 4.4$ & {$[4]$} \\
Bacteriorhodopsin & 5 & {$[15]$} \\
Large ribosomal subunit & 25 & {$[16]$} \\
Ovalbumin & $40 \times 50 \times 70$ & {$[4]$} \\
Bovine serum albumin & $50 \times 70 \times 70$ & {$[4]$} \\
Virus & $50 \times 90$ & {$[17]$} \\
Conalbumin & $50 \times 56 \times 95$ & {$[4]$} \\
Tobacco mosaic virus & $18 \times 18 \times 300$ & {$[3]$} \\
\hline
\end{tabular}

the vast complexity of the heat bath, yet large enough to posses the characteristic shape features required for specificity in interactions [18]. During the early 1970s it was recognized that biological structures can be viewed as molecular computers [19, 20, 21, 22]. At the same time the computational capabilities of chemical kinetic systems had been explored $[23,24]$ and the potential of molecules as circuit elements became apparent [25]. Subsequently many theoretical concepts have been developed, however practical realization of prototypes was slower than expected [26, 27] and at the time some authors adopted a rather pessimistic perspective [28]. Nevertheless, the study into artificial molecular computers continued [29, 30, 31] and laboratory prototypes for a variety of concepts have been demonstrated over the past years (detailed in section 3). 
Table 2: Gates with a single input

\begin{tabular}{l|l|l|c}
\hline Input & 0 & 1 & \\
\hline$G_{1}$ & 0 & 0 & constant 0 \\
$G_{2}$ & 0 & 1 & PASS, identity \\
$G_{3}$ & 1 & 0 & NOT, $\neg$ \\
$G_{4}$ & 1 & 1 & constant 1 \\
\hline
\end{tabular}

From an information processing perspective the keenly discriminating specificity of macromolecular interaction entails a marvelous pattern recognition capacity $[32,33]$ that supports computing based on the self-assembly of molecular data representations [34, 35]. In the past decade the practicality of such and approach has been demonstrated for minimal problem instances with self-assembly of short deoxyribonucleic acid (DNA) strands [9, 36].

Another route to molecular computing is based on the flexibility of macromolecular structures. Changes in the conformation, i.e., changes in the spatial positions of the atoms of a molecule that do not require disruption of covalent bonds, can be used to store and to process information. The protein bacteriorhodpsin is a particularly successful example of the application of this principle (c.f. sections 3.3 and 3.6). Part of the success of this protein can be attributed to its stability. It is worth pointing out, that the existence of a ground state and discrete energy levels insure that multiple copies of a molecule have and maintain identical properties. A water molecule, for example, that is a million years old is indistinguishable from a water molecule produced a millisecond ago. Fatigue or wear out as may be found in macroscopic structures [37] does not exist for individual molecules. Their parameters are constant throughout lifetime and thus enable the implementation of computation methods exploiting the subtle intricacy of molecular response [38].

Within the field of molecular electronics the development of polymer conductors and semiconductors formed a focus point early on [39, 40,41]. Initial predictions that polymer circuits would arrive within 20 years [42] turned out to be quite accurate, although the advantage of the circuits that are within reach now $[43,44,45]$ is not, as had been hoped, integration density and speed, but reduced fabrication cost per circuit-but not per transistor - as compared to conventional silicon-based circuits. The most immediate widespread application of molecular information technology is likely to arise from the use of low cost, low performance polymer circuits in ubiquitous computing. In these applications, so far, molecular materials are enlisted to implement essentially standard logic circuits.

\section{COMPUTING CONCEPTS}

\subsection{Current Paradigm}

The idea of a computing machine was conceived with the desire to free the mind from tedious, repetitious calculations as they occurred in the preparation of, e.g., astronomic and trigonometric tables. Before computers took over routine calculations, the methodology for performing these was based on standardized forms for collecting intermediate results and for tracking the incremental progress of the computation. The architecture and operation of current computers directly corresponds to this methodology.

The common computer is assembled from elementary pattern recognition units, the logic gates. Theses respond with characteristic binary output signals to signal patterns arriving at one or more binary input lines $[46,47]$. For the case of a single input there are four different ways to respond and accordingly in principle four gates could be constructed to realize these responses. The possibilities are shown in table 2 with each row showing the response for a gate labeled $G_{i}$. Input lines and output lines are assumed to be in one of two states which arbitrarily have been labeled 1 and 0 in the table. Two of the gates are indifferent to the input signal shown in the top row and respond with a constant output $\left(G_{1}\right.$ and $\left.G_{4}\right)$. Gate $G_{2}$ forwards the input signal without transforming it. From purely logic considerations such a PASS gate would appear to have no use. In practice however, it can serve to adjust signal delay or to reform a degraded signal. The gate $\left(G_{3}\right)$ that inverts the input signal is the most common single-input gate. Corresponding to the 
Table 3: Common logic gates with two input lines

\begin{tabular}{l|l|l|l|l|c|l|l}
\hline \multirow{2}{\text{InputA}}{} & 1 & 1 & 0 & 0 & & \multicolumn{3}{|c}{ Implementation } \\
Input B & 1 & 0 & 1 & 0 & Sym. & with NAND & with NOR \\
\hline NOT & 0 & 0 & 1 & 1 & $\neg$ & $\mathrm{A} \mid \mathrm{A}$ & $\mathrm{A} \nabla \mathrm{A}$ \\
AND & 1 & 0 & 0 & 0 & $\wedge, \cdot$ & $(\mathrm{A} \mid \mathrm{B}) \mid(\mathrm{A} \mid \mathrm{B})$ & $(\mathrm{A} \nabla \mathrm{A}) \nabla(\mathrm{B} \nabla \mathrm{B})$ \\
OR & 1 & 1 & 1 & 0 & $\vee,+$ & $(\mathrm{A} \mid \mathrm{A}) \mid(\mathrm{B} \mid \mathrm{B})$ & $(\mathrm{A} \nabla \mathrm{B}) \nabla(\mathrm{A} \nabla \mathrm{B})$ \\
XOR & 0 & 1 & 1 & 0 & $\oplus$ & & \\
NAND & 0 & 1 & 1 & 1 & $\mid$ & & \\
NOR & 0 & 0 & 0 & 1 & $\nabla$ & & \\
COMP & 1 & 0 & 0 & 1 & $\equiv$ & & \\
\hline
\end{tabular}

four possible gates with a single input line, there exit 16 gates with two input lines. Again some of these ignore all or one of the inputs, and only the logic operations shown in the six lower rows of table 3 are in practice useful. Combinations of logic gates can operate on more input lines. For example two AND gates can be combined to form an AND with three input lines $\left(\mathrm{I}_{1}, \mathrm{I}_{2}, \mathrm{I}_{3}\right)$, by first applying an AND gate to $I_{1}$ and $I_{2}$ and then applying and AND gate to the output of the first gate and $\mathrm{I}_{3}$.

A suitably chosen subset of the logic gates shown in tables 2 and 3 is sufficient to express arbitrary logic relationship between input variables. Such a set of gates (or logic operations) is called universal. An example of a universal set is the combination of NOT, AND and OR. To elucidate how this set of operations can implement an arbitrary logic function, one can imagine a table in which the $n$ leftmost columns represent the states of $n$ input variables. According to the $2^{n}$ possible state combinations of the input variables there would be $2^{n}$ rows in the table. A further column at the right would assign to each of the rows an output value. For simplicity of the present explanation (but without loss of generality) a single output line may be assumed. The filled-in table defines a logic gate with $n$ input lines and one output line. To compose this logic gate from the universal set mentioned above, it is sufficient to consider only rows for which the output is 1 . For each such row one employs NOT gates to invert any input signal that is in a zero state. One then combines the input lines and the inverted input lines with a cascade of AND gates. The output line of the final AND gate in the cascade is the output for the row and will be in a 1 state only if all input variables are in the state indicated by the corresponding row in the table. Finally the outputs of all $2^{n}$ rows are combined in a cascade of OR gates. The output of the final OR gate is the output for the logic function defined by the table. While the resulting circuit may not be the smallest possible, the construction illustrates that NOT, AND and OR gates can be combined to implement any arbitrary logic function, and shows that this set of gates is universal. It is possible to construct each of the three gates NOT, AND, and OR from NAND gates alone, and it is also possible o construct the three gates using only NOR gates, as indicated in the two rightmost columns in table 3. Consequently the NAND gate alone is sufficient to implement any other logic function, it is a universal gate. Also the NOR gate is universal.

To combine the gates into circuits two further building blocks will be required: a fanout element and an exchange element. The former replicates a single input line on two output lines, the latter exchanges the signal between two lines or two storage locations. In electronic implementations the former may just be a forking connector and the latter could be the crossing of two isolated connectors. Both are often implicitly assumed, but it is important to be aware of them for unconventional implementations [47]. If, for example, a signal is represented by a molecule instead of an electrical potential then the fanout element may need to replicate the molecule.

In implementations of the logic gates the physical dynamics of the operation will introduce a transient delay between the arrival of the input signal and the resulting output signal. Connecting the output of an inverter (a NOT gate) with its input would seem a logic contradiction if the action of the gates would be instantaneous. The switching delay, however, gives rise to oscillations if an odd number of NOT gate are connected in sequence with the output of the last gate fed back to the input of the first gate. An even number of NOT gates can be cascaded to form a bistable circuit, called a flip-flop, that will hold a set logic level indefinitely [48]. Similarly a 
chain of PASS gates will act as a delay line and temporarily retain the state of a previous input. As a consequence the output of a network of gates may not be solely a function of the input variables, but can also depend on the internal state of the network and thus on the history of inputs [49].

If the states of the network are discrete and the network consists of a finite number of components, then the network can only assume a finite number of states. Accordingly the network can only distinguish among a finite number of input histories. Such a processor is called a finite state automaton and is typically represented as a graph. The nodes in the graph correspond to states of the processor and the edges correspond to state transitions caused by input signals $[50,51]$. Any realizable digital computer is a finite state automaton, although often with a vast number of states.

The success of digital electronic circuits in implementing such automata has been attributed to several key factors common to relays, vacuum tubes, and transistor technology [52]:

- Tolerance to variation in component parameters arising from production, aging or environmental effects

- Resilience with regard to signal degradation and noise

- Restoration of output through high gain

- Ability to interconnect

It is interesting to note that historically the speed of individual components was not as important for the success of a particular technology as low power dissipation. The latter permitted high integration density and thus provided better overall system performance based on the slower components [53]. The scaling of electronic logic devices proceeded fairly predictable for the past 30 years with an essentially unchanged operational principle and device structure, and another decade of scaling seems physically feasible, although the economic limit of scaling may be closer $[54,55]$.

Computers excel in the tasks for which their concept was originally intended. Nevertheless many interesting information processing problems appear to be out of reach of our current computing devices. The ultimate limitations of computing have been discussed from theoretical and physical perspectives. At the heart of the study of theoretical limits on computability lies the notion of 'effective computation'. An effective computation can be carried out by a machine that executes precise instructions without any need for intuition, innovation, or guessing.

Turing [56] suggested a machine model that essentially comprises a finite state automaton coupled to an unlimited memory and permitted to operate without time restriction. This model has been shown to be equivalent to several independent formulations of effective computation by Church, Kleene, Post, and others [57]. It is now generally believed that all formulations of effective computation are equivalent and that anything that in principle can be computed can also be computed on Turing's machine. A machine capable of carrying out any computation is called a universal machine. Such machines are hypothetical constructs, because they cannot be limited by finite memory. The central result in computability theory is the proof that problems do exist that cannot be computed on a universal machine despite its unlimited resources. It is worth noting that universal machines can be surprisingly simple. Minsky offers a Turing machine design with a seven-state finite state automaton. Similarly a processor with a unlimited random access memory (RAM) needs an instruction set of only two instructions ('increment' and 'decrement with conditional jump') to be capable of computing anything that is computable $[50]$.

With this in mind it does not come as a surprise that even the early computing devices were finite memory approximations of universal machines. This universality led to many new directions of application. The all-purpose character of the computer together with the steady advance on the limits of memory space and CPU time shielded the underlying computing concept from challenge, and technological progress focused on quantitative improvements.

Within mathematics the concept of computability appears to be absolute. However, the idea of an effective procedure can not be defined mathematically, because any such definition would require a specific formal model of computation and hence undermine the absolute claim [58]. The broad scope of what is meant by 'effective procedure' can be extended beyond the boundaries 
of mathematics into physical reality. The border is crossed in the strong version of the ChurchTuring Thesis that can be stated as: any real-world process is effectively computable. A corollary is, that no physically realizable system (e.g., the human brain) can compute anything that could not in principle be computed on the present day computers, given enough memory and time.

The comparative capabilities of actually realizable systems shows a picture quite different from that yielded by a comparison of the ultimate theoretical limitations of their concepts. If effective computation is identified with physically realizable dynamics, then computability becomes constrained by physics. The velocity of light is the ceiling for the distribution of signals. Heisenberg's uncertainty principle limits the speed with which system states represented by energy differences can be determined. The physical upper bound on the amount of information processed within one second by a processor with a mass of $1 \mathrm{~g}$ has been calculated by Bremermann $[59,60,61]$ to be about $2 \times 10^{47}$ bit.

Physical limitations on computation arise also from the need to represent information by physical degrees of freedom and the resulting thermodynamic effects caused in the course of computation [37]. Instead of imposing a predefined logic behavior on the dynamics of the computing system, the course of computation can be driven by physics itself [35]. If the strong Church-Turing Thesis does hold, the class of problems that can be solved in principle with computation driven by physics will be the same as the class of problems that can be solved with formal systems. Then, in principle, every computable problem can be solved some day using the conventional computing paradigm, assuming open ended increase in memory capacity and processing speed [50]. But this does not mean that the current kind of computing is the suitable tool for all information processing problems. In application areas that do not resemble the calculation of tables, present-day computers have a hard time exhibiting satisfactory performance. Do alternatives exist?

\subsection{Unconventional Computing}

With the prevailing success of the common general purpose computer information processing became almost synonymous with digital computing. During the early days of computer science, however, the term 'computer' referred to a wide range of information processors [62], ranging from mechanical function generators to hydraulic equation solvers, and included human beings [63]. For the consideration of qualitatively new types of information processing devices it is helpful to return to this broad view of computing: A computer is a system that starts from a state which encodes a problem specification and changes, following the laws of nature, to a state interpretable as the solution to the problem [64]. Following this definition, a system trivially computes its own behavior. Any physical dynamics can usefully be employed for information processing if an interpretation is found to transcend the question of the system's own behavior. The physical dynamics can be carefully tailored to closely adhere to a formal model of computation. The engineered constraints in the electrodynamics of the common digital computer are a case in point. Or the physical dynamics can be used in a direct way by selecting encodings and interpretations. The use of a lens to implement a Fourier transform is an example. The latter approach often provides a simple implementation for an intricate input-output mapping. This, however, comes at the price of narrowing the application domain [65]. Developing concepts of computation that do not impose such rigid constraints on the physical dynamics of the implementation as to abrogate its efficiency [66], yet are flexible enough to be of general use is a challenge. In the context of molecular information processing the search is for paradigms capable of exploiting the specific characteristics of molecules rather than requiring the molecules to conform to a given formal specification.

A number of unconventional computation schemes may serve as stepping stones towards this goal. Cellular Automata, for example, are arrays of usually identical finite state automata. The inputs to each automaton are the current states of its neighboring automata. A hypothetical infinite array of suitably constructed finite state automata could operate as a universal computer [67]. Conceivably a cellular automaton can be implemented with individual molecules acting as finite state automata. The automata states could be represented by charge distribution within the molecule and electrostatic interaction among the molecules could serve to couple neighboring automata [68]. Molecular cellular automata may have the potential of providing an array fine grained enough to consider it essentially as a continuous computing medium [69]. The requirement of a highly symmetrical and homogeneous structure limits the applicability of the 
A

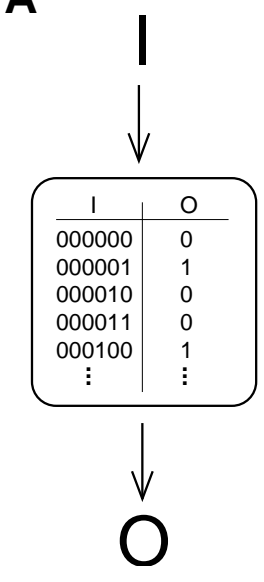

B

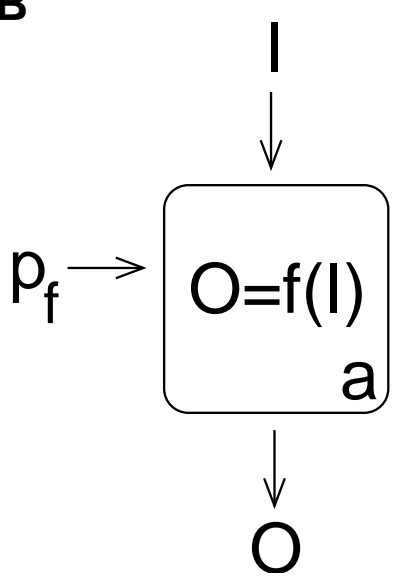

Figure 1: Communicating a desired input-output map to a machine. The input-output map can in principle be thought of as a potentially very large lookup table that associates an output response with every input that can be discerned by the machine (A). For $n$ bit input patterns I and a $m$ bit output $(\mathrm{O})$ response the, number of possible maps is $2^{m 2^{n}}$. To implement an arbitrary one of these maps on a quasi-universal machine, the mapping $f$ has to be specified by the program $p$ with respect of machine architecture a $(\mathbf{B})$. Selecting an arbitrary map from the set of possible maps may require a specification of length: $\log _{2}\left[2^{m 2^{n}}\right]=m 2^{n}$. Even for moderate pattern recognition problems (e.g., classifying low resolution images) the program length required for most mappings is impractical [38, 73].

cellular automata computing. However, concepts for massively parallel computing inspired by the self-organized collaboration of biological cells in organisms are under development. Amorphous computing, for example, assumes a large number of identically programmed computing devices in arbitrary positions and orientations. The computing devices interact through short range communication and are explicitly assumed to be unreliable [70]. This concept relaxes two constraints imposed by conventional computing architectures. The components do not have to be arranged following exactly a predetermined spatial structure and the components do not all need to perform according to narrow specifications. The amorphous computing concept is an important step towards broadening the material basis of computation to include molecular and nano materials. However, it does suppose that the elementary devices are programmable and global functionality emerges from handmade programs executed by the elementary devices. This assumption limits the efficiency as well as the general usability of the inherent parallelism $[71,72]$.

Programming invites another restriction. The length of the program is limited by the state space of the device and the capacity of the programmers. Both can be exhausted rather quickly even with relatively small problems as is illustrated in Figure 1). As a consequence conventional computing architectures are in practice restricted to the implementation of highly compressible input-output maps [64]. The set of compressible maps is a small subset of the potential inputoutput functions - most behaviors cannot be programmed. Whether the incompressible and thus inaccessible mappings are useful is an open question. In the light of the ability of organisms to cope with complex ambiguous pattern recognition problems it appears likely that input-output mappings of limited compressibility can be valuable in practice. The picture painted so far optimistically assumed no two different programs implement the same input-output map. In practice, however, the mapping of input into output is achieved by decomposing the transformation into a series of sequential elementary information processing operations. Information processing is essentially selective dissipation of information and each operation entails a, possibly delayed, loss of information [74, 37]. Now, if the transformation of input signal patterns is decomposed into a large number of operations, all information pertaining to the input may be dissipated in the processing sequence. And so it may happen that the output response will be independent of the input and thus constant $[75,76]$. This further reduces the number of input-output maps accessible through programs. 
One way to overcome this problem would be specialized hardware with only a limited application domain. In the extreme case it may be useful to build an instance machine, i.e., a specialized computer architecture dedicated to solving a single problem instance. This opens up several design degrees of freedom [77]. In particular the upper bounds for the number of state transitions individual components have to support can be very low. Materials and procedures that would not come under consideration for conventional computing devices may find niche applications in instance machines.

Another way for implementing complex input-output maps is to avoid programming. Programmability is not a prerequisite for the realization of information processing systems as is exemplified by the enviable computing capabilities of cells and organisms. Artificial neural networks provide a technological example of non-programmed information processing [72]. They trade an effective loss of programmability for parallel operation. This approach could be taken much further, however. As programmability has essentially been relinquished, the individual network nodes do not need to perform identically. Inhomogeneity and context-sensitive components can be introduced to increase the computational power and the efficiency the network [66, 78]. These steps abrogate present training algorithms for artificial neural networks. At the same time however, the increased dimensionality enhances the evolvability of such networks. The evolutionary paradigm of using the performance of an existing system as an estimate for the expected performance of an arbitrarily modified version of the system can cope with the complexity and inhomogeneity of architectures based on context sensitive components. In fact it is particularly effective in this domain [79].

In general, however, evolving highly complex input-output maps from scratch may take so long as to be impractical. It is here where the concept of 'informed matter' [80], i.e., molecules deliberately designed to carry information that enables them to interact individually, autonomously with other molecules, comes into play. Already the level of reaction kinetics supports considerable computational capabilities [81] and fast algorithms for simulating kinetic computing schemes are available [82]. Abstract concepts in artifical chemistry [83] go beyond the kinetic level and encompass supramolecular interactions. The developments in supramolecular chemistry $[84,3,80]$ point towards the possibility of implementing computing schemes developed as artificial chemistries with bona fide molecules. Conceivably this will enable orchestrated self-organization to arrive at time scales that is practical and thus enable physics driven architectures.

\section{IMPLEMENTATIONS}

\subsection{Carbon-based Semiconductors}

\subsubsection{Polymer Electronics}

Current information processing devices are generally based on networks of electronically actuated switches. The type of switch most commonly employed is a variant of the field effect transistor (FET) first described by Shockley [85]. Fig. 2 depicts a typical configuration of an insulated gate FET. If the electric potential at the gate electrode is zero, the current flow between source and drain electrodes is low. On application of a potential at the gate electrode, a charge is induced at the insulator-semiconductor interface. The charge provides carriers in the semiconductor layer and enables an increased current flow between the source and drain electrode. Consequently the gate can be used to switch the source-drain current on and off. The characteristics of the transistor can be modified by altering the geometry of the gate. In conventional FETs the insulating layer can be formed by metal oxide that separates the gate electrode from an inorganic semiconductor such as Si, a technology known as metal-oxide semiconductor (MOS).

Within two decades of the demonstration that an organic polymer (polyacetylene) can also serve as semiconductor in a FET $[86,87]$ carbon-based FETs have reached the verge of commercialization [88]. However, due to their low mobility (i.e., charge carrier velocity per unit electric field) they cannot compete with the speed of inorganic semiconductor switches. It has been suggested that low mobility could potentially be overcome by reducing the organic semiconducting layer to a single macromolecule [88]; thus eliminating the need for charges to hop from molecule to molecule while traveling between source and drain.

The use of an individual molecule as active material in a transistor would be a significant step toward single-molecule devices [89]. As early as 1974 Aviram and Rattner suggested that a 


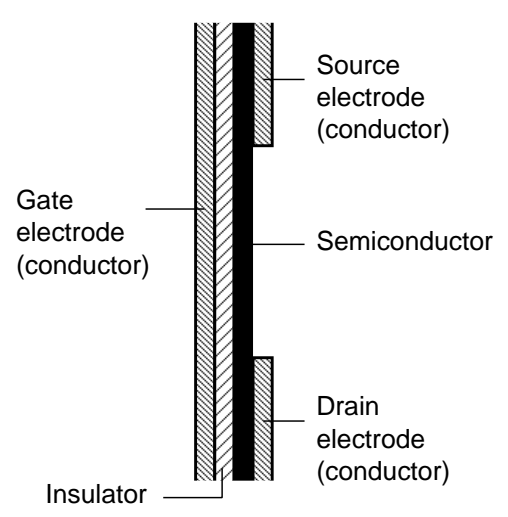

Figure 2: Conducting, semiconducting and insulating materials are required to assemble an insulated gate FET, but the configuration is relative simple and well suited for low-cost production.

hypothetical molecule consisting of an electron donor part connected by a saturated $(\sigma-)$ bridge to an electron acceptor would function as a single-molecule diode [25].

Rectification has been achieved in a multilayer stack of oriented molecules [90] that was prepared by the Langmuir-Blodgett (LB) technique [91, 92]. Subsequently a LB monolayer acting as photodiode was obtained [93]. This result indicated that single molecule devices are indeed realizable. The synthesis of a molecule that acts as a rectifier, however, turned out to be far more arduous than what had been anticipated and an unimolecular rectifier was achieved only recently [94, 95]. Rectifiers suffice to implement logic AND and OR gates [96]. But, as noted in section 2, for implementing the complete set of logic functions, inverters are required in addition to AND and OR gates. Simulations suggest that individual Fullerene $\left(\mathrm{C}_{60}\right)$ molecules could potentially be used to build inverters and furthermore that the NOR operation may possibly be implemented with two $\mathrm{C}_{60}$ molecules [97]. NOR gates alone would allow for the realization of all logic functions as can be seen from the rightmost column of Table 3 . Here may be mentioned that $\mathrm{C}_{60}$ is of interest not only for single molecule devices but also in bulk as n-channel semiconductor material, [98] especially because most known organic semiconductors are p-channel materials.

The rising commercial interest in molecular electronic devices focuses currently on the use of bulk material, not individual molecules. Slow switching speed concomitant with the use of bulk organic materials are offset by the potential for low-cost fabrication, large circuit area, mechanical flexibility, light weight, and production processes compatible with temperature sensitive materials such as transparent plastics. To realize these possibilities, ideally all layers of the FETs (cf. Fig. 2) should be implemented with molecular materials. Recently de Leeuw and co-workers showed that this is feasible by building integrated circuits in which the insulating, conducting and semiconducting layers are polymers [43]. These circuits comprise several hundred FETs connected as 15-bit programmable code generators. Versions fabricated with pentacene as semiconductor are reported to operate at up to $100 \mathrm{bit} / \mathrm{s}$ and to show negligible performance loss after exposure to air for over a month [44]. The latter is encouraging since many organic semiconductors with otherwise interesting properties degrade in the presence of oxygen through oxygen-doping and oxidation. Encapsulation of the circuits may in principle be used for obtaining reliable devices with adequate shelf and operational life time. For low-cost fabrication, however, it would be desirable to have organic materials available that are not overly sensitive to open air and impurities.

Solubility is another property sought in organic semiconductors. Solution-processable materials which can be patterned by printing and printing techniques are ideal to facilitate cheap production [99, 100, 101, 102]. The demonstration [45] of a ring-oscillator produced from soluble polymers that is capable of operating at $106 \mathrm{kHz}$ is therefore a significant step toward the commercial application of organic FETs.

However, so far no soluble n-channel material has been found [103]. Consequently printed circuits in complementary technology (i.e., the organic equivalent of CMOS) are not yet feasible. In CMOS technology complementary p-channel and n-channel transistors are combined in series to yield circuits with low power requirements (Fig. 3). Organic complementary circuits have been 


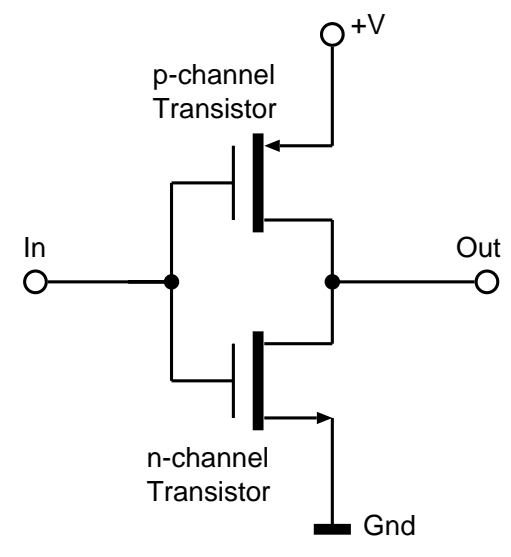

Figure 3: Logic NOT (inverter) in CMOS technology. Power is only consumed during a state change, since for both input states (high and low) only one of the two transistors will be conducting.

fabricated with photolithographic patterning [104]; achieving the same with printable materials is highly desirable to allow of low cost power supply options. We note however, that the low power consumption of complementary circuits is largely due to the small number of gates that are active at any given point in time (see section 2 above). Architectures that rely on concurrent operation of the active elements would considerably reduce the CMOS advantage.

A strong drive for the commercial development of polymer-electronic is the immediate and potentially very large market for radio frequency identification (RFID) [105]. Transponder tags for RFID are comprised of an antenna and a low complexity circuit that is powered by incoming radio waves. Upon activation the circuit broadcasts a unique identification code that can be matched to a database to track the movement of the tag in space and time. Cheap circuits of sufficient speed to operate at the established RFID frequencies would make the automatic identification of low cost items economically viable. The performance limitation of organic semiconductors are addressed not only by seeking new materials, but also with new circuit designs. Baude et al. proposed an AC powered inverter, thus eliminating the need for a high quality rectifier component [106]. They demonstrated a pentacene-based implementation of an AC powered 7-stage ring-oscillator, but with only 1 bit modulation [107]. It remains to be seen whether identification codes with a useful bit length can be implemented with the AC powered design. Undoubtedly, the adaptation of the circuit design to the specific properties and limitations of the materials is a promising path.

The potential advantage of low-cost, large circuit area and fabrication methods compatible with heat sensitive materials indicate a promising future for organic electronics. Current research focuses on utilizing the large circuit area and low processing temperatures (suitable for flexible and transparent plastics) in displays [108]. It appears likely that these features will also enable numerous biomedical and biotechnology applications based on integrating organic electronic circuitry with biomolecules and even micro organisms $[109,110]$. The shelf-life and operation time of such bio-electronic hybrid systems may be limited by the integrated bio-compounds or cells and thus offer a niche application in which materials with limited stability can usefully be employed.

\subsubsection{Carbon Nanotubes}

The thermodynamically stable crystalline from of bulk carbon is graphite with strong covalent bonds in a two-dimensional plane forming sheets with a hexagonal lattice structure. For an amount of carbon small enough to be affected by the finitude of its atom number it is energetically favorable to give up the planar structure in order to close dangling perimeter bonds with itself [13]. Closed cages result. The $\mathrm{C}_{60}$ structure mentioned in the previous section is an example. A variety of sizes of globular shells starting with $\mathrm{C}_{28}$ have been found, commonly termed Fullerenes. One can now imagine to roll a narrow strip of a two-dimensional graphite sheet into a tube such that the carbon atoms along the two long edges of the strip form bonds. Tubes, capped with half a Fullerene on either end can be imagined [111] and do indeed occur [112]. Single-walled macromolecular tubes have diameters from 0.75 to $13 \mathrm{~nm}$. A diameter of $1.2 \mathrm{~nm}$ and a length 


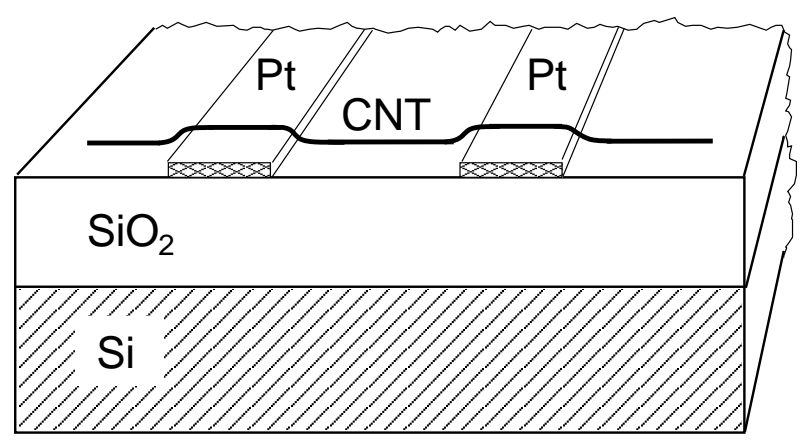

Figure 4: Carbon nanotube field effect transistor. A single walled semiconducting carbon nanotube (CNT) contacts two platin electrodes (Pt). A semiconducting Si substrate serves as gate electrode insulated from the platin electrodes and the CNT by $300 \mathrm{~nm}$ of $\mathrm{SiO}_{2}$. This thick insulating layer results in less than unit gain (0.35). The insulating layer can be reduced to a few nm, permitting about unit gain. After [114].

of several $\mu \mathrm{m}$ is typical $[13,14]$. A slight longitudinal shift can be introduced when matching the dangling bonds along the edges and will cause a helical twist in hexagonal lattice of the tube walls. Depending on diameter and on the degree of helicity the electronic properties of the tubes can vary from conducting to semiconducting [113].

Tans et al. demonstrated a FET based on a single-walled semiconducting carbon nanotube [114]. The device, illustrated in Fig. 4, operates at room temperature and a conductance change of about six orders of magnitude for a gate potential of $10 \mathrm{~V}$ was reported. The contact resistance, however, was high and a resistance of $1 \mathrm{M} \Omega$ was measured for the conducting state. Furthermore the gain of this transistor was below unity and hence the transistor would not be suitable in circuits where it would need to drive other transistors.

As mentioned above, carbon nanotubes can be pictured as rolled hexagonal lattices. The end caps however do contain six pentagons among the hexagons [13]. Owing to the higher reactivity of pentagons compared to hexagons the end caps of nanotubes can be destroyed by oxidation. This provides free bonds on the nanotube ends that can be functionalized by covalently linking the tube to another molecule. It is possible, for example, to link nanotubes to single stranded DNA $[115,116]$. The self-assembly properties of complementary DNA strands, further discussed in sections 3.5 and 4.3, can then be exploited for attaching the DNA-functionalized nanotube at a specific location of a solid support or for attaching the nanotube to other molecules, including other nanotubes. Particularly attractive are noncovalent methods of attaching macromolecules to single-walled carbon nanotubes, because these methods leave the extended electronic structure of tubes intact. Noncovalent binding can be accomplished by wrapping of the tubes [117] or by using a highly aromatic linker that adsorbs irreversibly onto the hydrophobic surface of the tubes $[118,117]$.

Keren et al. devised an original strategy to form electric contacts with a carbon nanotube [119]. They self-assembled a semiconducting nanotube in the center of a $16 \mu \mathrm{m}$ DNA molecule. The DNA molecule was then coated with gold except for some area along the tube which was protected by a DNA binding protein. They used this assembly to implement a nanotube FET similar to the type shown in Figure 4, but with the DNA-templated gold wire replacing the platinum contacts on either side of the nanotube which is itself bound to the DNA molecule.

Dekker and co-workers found that nanotube FETs with an on-off ration of $\geq 10^{5}$ and a gain of 10 can be achieved by using a microfabricated aluminum wire as gate electrode which is insulated from the nanotube by its native layer of $\mathrm{Al}_{2} \mathrm{O}_{3}$ [120]. These transistors are of sufficient quality to permit multi-transistor circuits. Circuits with up to three nanotube FETs have been produced, including the universal NOR gate (cf. table 3), a two-inverter flip-flop as 1 bit static memory cell, and a three-inverter ring-oscillator (cf. section 2.1).

Much attention has been bestowed on carbon nanotubes since Iijima's pointed out the helical structure of nested, 'multi-wall', tubes [112]. The field develops rapidly [121, 122] and has only been touched upon here. Increasing control over the fabrication of nanotubes and concomitant wider availability of tubes with desired characteristics furthers the theoretical understanding of the phenomena associated with them. Key issues that need to be addressed are the con- 
tact resistance and the formation of multi-device circuits without a need for positioning each individual nanotube manually. The possibility to functionalize carbon nanotubes with macromolecules raises the prospect of tubes with new and tailored characteristic becoming available. The excellent reviews of Ajayan [13] and Terrones [14] are recommended for further information.

\subsection{Molecular Switches}

The quest for molecular transistors discussed in the previous section is largely driven by their potential as building blocks for switches. It may be recalled (from section 2) that current computing technology is build on vast networks of simple switches that implement logic operations as well as memory. Direct realization of a high-quality molecular switch rather than through interconnection of molecular transistors is therefore an attractive idea. Early considerations of switching in molecular systems showed that bistable kinetics can be realized with bulk chemical reactions [23]. In this section, however, the focus is on individual molecules or supramolecular systems that can act as switches.

If a very large number of switches is available and can be suitably connected then even a switch that sustains only a single switching cycle may be of technical interest. Such devices could be used to store input-output mappings in enormous lookup tables, essentially replacing active computation with a vast amount of memory.

A molecular switch that can be opened irreversibly has been demonstrated with rotaxane molecules [123]. The rotaxane comprises two motives, a molecular ring and a v-shaped molecule. The ring is interlocked with one arm of the v. Large molecular groups cap the ends of both arms of the $\mathrm{v}$-shaped molecule and prevent the ring from sliding off. A monolayer of these rotaxane molecules was sandwiched between two electrodes (terminal- $\mathrm{Al} / \mathrm{Al}_{2} \mathrm{O}_{3}-\triangleleft-\mathrm{Ti} / \mathrm{Al}$-ground). At a bias voltage below $-1 \mathrm{~V}$ the device is conducting. A positive bias of 0.7.- $0.9 \mathrm{~V}$ leads to oxidation of the rotaxane and permanently opens the switch. Diode-type [124, 125] AND gates and OR gates, and write once configuration by irreversible switching have been reported [123].

Reversible redox-controlled switches have also been developed [126]. [2]Catenanes are molecules comprised of two interlocked molecular rings. Oxidation of a [2]catenane molecule results in a rotation of one of the rings relative to the other (Fig. $5 \mathrm{~A}$ ) and yields a conducting state. The catenane can be reset to the open-switch conformation by applying a reducing potential. However, the switching effect in the [2]catenanes is less pronounced than in the irreversible rotaxanes. Pseudorotaxanes, a family of molecules where the two sliding molecular motives are not interlocked, but self-assemble may point a way to more effective reversible molecular switches (Fig. 5 B).

The researches of Tour and collaborators appeared to indicate that conjugated phenylene ethynylene oligomer derivatives can serve as conductance switches in electronic circuits [127, 128]. Theoretical analysis attributed the observed negative differential resistance of the molecules to electron adsorption [129]. On account of the conductance switching in molecules individually addressed by means of scanning tunneling microscopy it has been concluded that the switching is not an electrostatic effect of charge transfer, but due to conformational switching in the molecule [130]. Measurements taken on individual molecules insulated by inserting in a self-assembled alkanethiol monolayer confirm a negative differential resistance for single molecules [131].

In the light of a recent observation that switching behavior can occur in some experiments even in the absence of molecules, [132] the work on conductance switching with phenylene ethynylene oligomers appears at present controversial. Perhaps, electromigration of contact metal first forms conducting nano filaments at low voltages and these then break at higher voltages [132]. It remains to be seen to what degree contact effects, electromigration, metal filaments, or other artefacts have affected the experiments on molecular electronic switching that have been reported in the literature.

Molecules acting as electronic switches would most naturally interface with existing integrated circuits, but various other signal representations, including temperature, pressure [133], and spectroscopic methods [134] have also been investigated. An important class of molecular switches work with light signals. The potential of bistable molecules that can be toggled by light for information technology was recognized early on. Hirschberg, who coined the term "photochromism" for light-induced reversible spectral change [135], suggested already in 1956 the possibility of photochemical memories [136]. When a photochromic molecule in its thermodynamically stable state absorbs light, it can be transformed to a new state with a different absorption spectrum. 
A

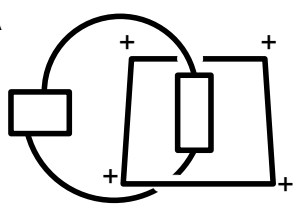

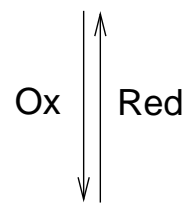

TTF

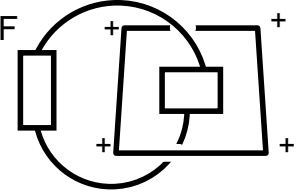

B

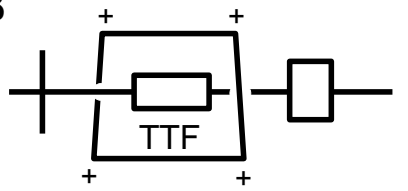

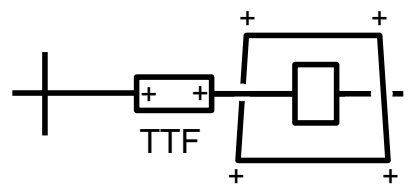

Figure 5: Bistable molecules can function as redox-controlled molecular switches. The left panel (A) shows the two interlocking rings of a [2]catenane molecule schematically. A positive voltage bias oxidizes a tetrathiafulvalene group (TTF) in the crown ether ring of the [2] catenane molecule. Electrostatic repulsion rotates the crown ether ring and closes the switch (bottom). Removal of the voltage bias will reduce the TTF. The [2]catenane molecule, however, will stay in the closed-switch conformation. A negative bias is required to return the crown ether to its open-switch position (top). Solid state switches have been prepared from the [2]catenane and the currents in the open and closed state were found to differ by factor of about three [126]. A supramolecular pseudorotaxane based on the same molecular motifs as the [2] catenane is schematically shown in panel (B). A positive bias ionizes the TTF group, whereupon Coulomb forces drive a reversible shift between the two molecular components. The pseudorotaxane exhibits a sharp change in conductance and hysteresis characteristics that indicate the potential to fabricate improved solid state switches. After [126].

Thermal energy or again irradiation with light returns the molecule to its thermodynamically stable state. By means of the different absorption of the two states it is possible to optically read out the state of the molecule. The next section presents an example for photochromism in more detail.

Optical output from molecules does not need to be conveyed by a change in the molecule's absorption. Fluorescence is an attractive option, because it can be quenched very effectively and thus affords large on-off signal ratios. The quenching of a fluorophore can be achieved photochemically through transfer of an electron to or from the fluorophore. The photoinduced electron transfer (PET) process that turns off the fluorophore can be affected by the chemical environment of the group that provides (or accepts) the transferred electron. This principle underlies a series of molecular switches that can be pictured as consisting of three modules, a receptor, a spacer and a fluorophore [137]. A fluorescent AND logic gate with chemical input signals in form of sodium and proton ions was devised along these lines [138]. Figure 6 illustrates the concept. The modular construction of theses molecules provides considerable freedom for design. Fluorophores can be chosen according to desired excitation and emission wavelength and receptors can be adapted to the input signals. By now the collection of available fluorescent switches includes XOR, NAND and NOR gates among more idiosyncratic logic tables [139].

Another approach is the use of ion dependent complex formation to shift the peak intensity of fluorescence emission of a bulk solution in response to input signals coded as ion concentration [140]. It is worth pointing out here, although we will return to this issue in section 4, that there is so far no mechanism in sight that could support arbitrarily chosen connections among such gates. Nevertheless molecular information processing in its simplest form may soon find applications for combining signals from several molecular sensors to a single output signal. For example, one can imagine an optical signal indicating a critical state in an intracellular compartment such as the cell nucleus. 


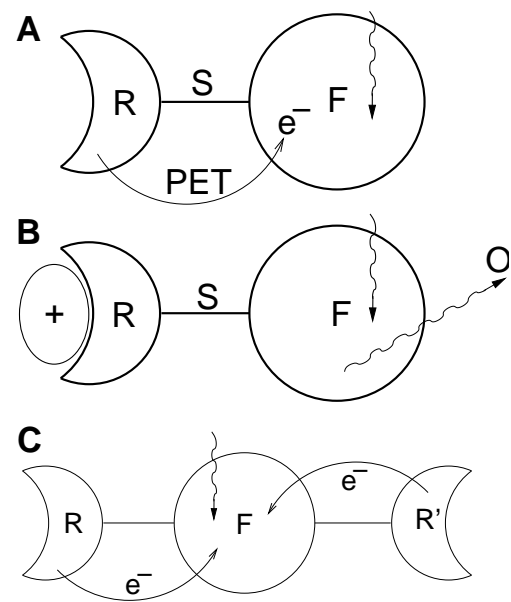

Figure 6: Molecular fluorescent switch comprised of a receptor (R), spacer (S) and fluorophore (F). Photoinduced electron transfer (PET) from the unoccupied receptor quenches the fluorophore, the switch is off; panel A. A cation signal (+) bound to the receptor prevents the PET and a fluorescence signal is emitted, the switch in on; panel B. If two receptor modules are coupled to a fluorophore the molecule behaves like a logic AND gate. Only if both receptors are occupied the electron transfer to the fluorophore ceases and a fluorescence signal appears. After [137].

In the fluorescence switches discussed above electrostatic influences on the receptor biased the competition between fluorescence and PET. Another mechanism for turning the quenching of a fluorophore on and off would be to change the spatial separation between fluorophore and quencher. Some biological macromolecules undergo large but well defined change in shape in response to interaction with other molecules or ions. By judiciously linking a fluorescent dye and a quencher to such a macromolecule the conformational change can give rise to an optical output signal. The folding of self-complementary DNA and RNA single strands is among the most predictable conformational changes in large macromolecules. A strand in which the nucleotides at one end are complementary to the nucleotides at the other end will form a hairpin like structure in which the two complimentary ends are aligned. (Fore more detail on the self-assembly of DNA strands see section 3.5.)

Tyagi and Kramer attached on one end of a partially self-complimentary DNA strand a fluorophore and on the other end a quencher [141]. The resulting molecular beacon folds into the hairpin structure and by so bringing the quencher and fluorophore moieties close together turns its own fluorescence off. If this molecular beacon encounters a single stranded DNA molecule that contains (or consists of) a sequence complementary to the beacon's sequence then the beacon opens and fluorescence is turned on (Fig. 7).

This principle was extended by Simmel and Yurke to a three state switch that can be cycled through its states by adding appropriate single stranded DNA molecules [142, 143]. Switch operations occur on a time scale of minutes, with a tradeoff between closing speed and opening speed. The three output states are distinguished by different levels of fluorescence intensity. The variation in intensity are achieved by drawing excitation energy from the fluorophore through fluorescence resonance energy transfer (FRET) to the quencher. The amount of energy that is transfered depends on the distance between the fluorophore and the quencher. In each of the three conformational states this distance is different and consequently results in a distinct output intensity. Note, that the principle of the above mentioned DNA based switches allows for the discrimination of a large number of sequence signals with essentially a single switch design. FRET can also supply a fluorophore with excitation energy. This fact was used in conjunction with DNA to implement logic gates [144]. However, the design of these gates does not exploit the selectiveness of DNA self-assembly and the approach is best compared to the aforementioned fluorescence switches based on organic molecules. Reversible conformational changes have also been reported for double stranded DNA controlled with potassium and strontium [145]. In passing we can point out that fast conformational change within the supra-molecular ion channels in cell membranes functions as an important physiological switching mechanism for cellular 


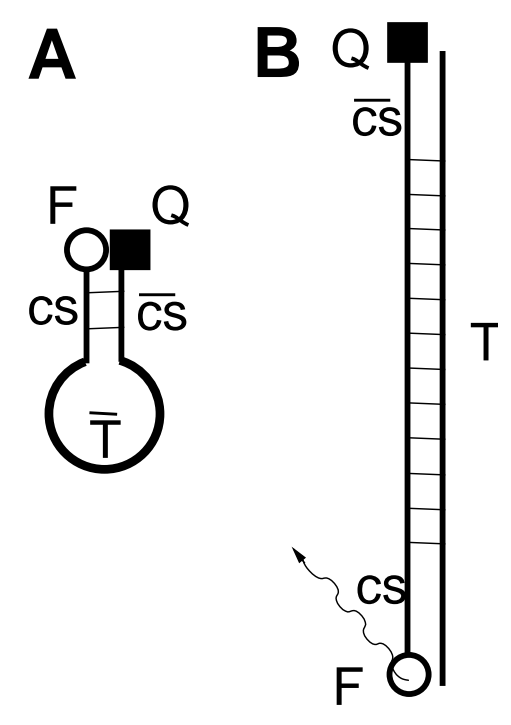

Figure 7: Molecular beacons signal the recognition of a specific DNA or RNA sequence [141]. The beacon consists of a piece of single stranded DNA with a sequence part at one end (cs) being complementary to the corresponding part $(\overline{\mathrm{cs}})$ at the other end. The two complementary sequences surround a sequence $(\overline{\mathrm{T}})$ complementary to the target sequence to be recognized. One end of the sequence carries a fluorophore $(\mathrm{F})$, the other end a quencher $(\mathrm{Q})$. The DNA strand folds unto itself and prevents fluorescence by bringing the quencher close to the fluorophore; panel A. Binding of a DNA strand containing the target sequence $(\mathrm{T})$ opens the beacon and separates the quencher from the fluorophore. The latter optically indicates the binding of the target sequence; panel (B).

regulation and electric signaling, for example, for the generation and propagation of action potentials in neurons $[146,147]$.

Molecular switches play an important part in gene regulation networks and can be used to turn the transcription of genes on or off $[7,8]$. Overlapping protein binding sites on DNA have been suggested for the realization of flip-flop switches that would result in sharp on-off transitions [148]. The cellular response to switching in gene networks is intrinsically slow and is not a path for advancing computing power. But it is of value as a research tool for experimenting with dynamically modulated gene expression [8] and harbors great potential for applications controlling individual cells. The latter could be used, for example, to orchestrate microbes in environmental restoration tasks or to activate the production of a drug when a need for it is sensed by a combination of sensors. A step in this direction is described in section 3.7.

A problem of optical molecular switches is that the photo excitation required to read the switch state can lead to side reactions and therefore has the potential to alter to the molecule. Let us proceed now to a molecule that has proved to be exceptionally stable in this regard.

\subsection{Bacteriorhodopsin: a Photonic Automaton}

The first successful employment of biomolecules in artificial information processing architectures has been achieved in the field of optical computing. Optical engineers were familiar with the possibility that a biological material may surpass synthetic materials in technical applications: gelatin, made from the protein collagen, had a long-standing reputation in the production of photographic films. During the 1970s researchers at the Biophysical Institute at Pushchino (near Moscow) found that a bacterial protein can be processed into films with many properties desirable for holographic recording $[149,150,151]$. The protein used in these 'biochrom films' was bacteriorhodopsin (BR). It occurs as two-dimensional crystals in the cell membrane of Halobacterium salinarium, an archaebacterium adapted to a harsh environment. Growing optimally in salt $(\mathrm{NaCl})$ solution seven times more concentrated than sea water, the bacterium is also accustomed to high levels of ultra violet light as well as relative high temperatures [12]. When nutrients become scarce it turns to an elementary version of photosynthesis. A single 248-amino- 


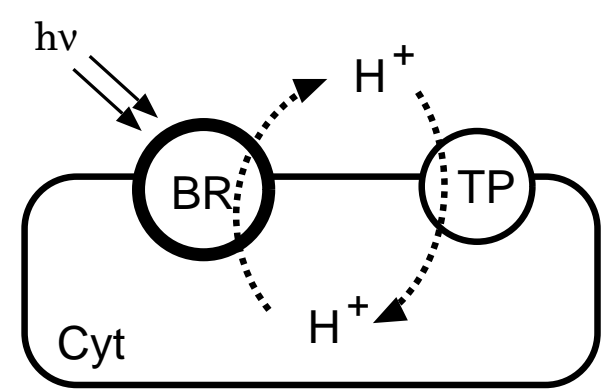

Figure 8: The protein bacteriorhodopsin (BR) is found in the cell membrane of Halobacterium salinarium. It functions as a light-driven pump, moving protons from the cytoplasm (Cyt) to the extracellular space. Thus, BR transduces light energy $(h \nu)$ into an electrochemical potential in form of a proton gradient across the cell membrane. This potential then powers transmembrane proteins (TP) that synthesize ATP (the 'energy currency' of cells) or serve as ion pumps.

acids protein, BR, serves as a simple, efficient, and robust solar power source (Fig. 8). BR uses light energy to pump protons from the inside of the bacterium across the membrane to the extracellular space. Thus it generates an electro chemical potential across the cell membrane which in turn is used as a driving force for various transmembrane proteins. A photocycle (i.e., a pumping cycle) takes $10 \mathrm{~ms}$ and comprises a series of conformation changes of the protein. At least eight conformation states can be identified in the physiological photocycle, more if non-physiological conditions are taken into account [152]. Absorption of light by a chromophore located in the interior of BR initiates the photocycle. The first conformation change, the isomerization of the chromophore, is reported [153] to take 500 fs. It is followed by a sequence of BR states, each with a characteristic spectrum and state life time [154].

Conformational states with distinct absorption maxima in combination with the possibility of photonic state switching are the basis for BR's great technical potential. Fig. 9 depicts conformation states and photo inducible transitions that are particularly relevant for information processing and storage applications. The large spectral shift for $\mathrm{B} \leftrightarrow \mathrm{M}$ transitions of $\approx 160 \mathrm{~nm}$, and state switching with wavelengths in the visual light range are notable. BR is at the same time highly photosensitive (the quantum efficiency for both the $\mathrm{B} \rightarrow \mathrm{M}$ transition triggered with green light and the $\mathrm{M} \rightarrow \mathrm{B}$ transition triggered with blue light is above $60 \%$ ) and exceptionally stable against thermal, chemical and optical degradation. In combination these features are unrivaled by any synthetic material [156]. Of great importance for technical applications is the exceptional stability of BR. Hampp's group found that BR films can undergo over a million switching cycles and consequently are well suited for real-time optical computing at video frame rate [15]. The very fast initial conformation change of BR permits optical recording on picosecond time scale. Furthermore, BR films support an optical resolution in excess of $5000 \mathrm{lp} / \mathrm{mm}$ and are routinely fabricated with over $80 \mathrm{~cm}^{2}$ apertures [155].

The BR molecule has been studied extensively for over thirty years both from the perspective of its physiological function and also as material for technical applications $[158,155]$. Optimized over billions of years by natural evolution BR exhibits characteristics of what has been termed an 'intelligent material'. As a single molecule it integrates the ability to sense environmental conditions, to process the sensory information, and to adapt its function accordingly [159].

The relative detailed understanding of the molecular level mechanisms of BR [160] enabled reverse engineering of one of nature's nanotechnology solutions. As a consequence BR became the first biomolecule that has been tailored for technical applications $[161,15]$ by means of genetic engineering [162]. By now numerous BR mutants have been created. Two variants exhibit significant advantages over wild-type BR and hence became popular as optical materials. The first variant is called BR-D96N to indicate the substitution of a single amino acid (aspartic acid $\rightarrow$ asparagine) at position 96 of the amino acid chain that constitutes the BR protein. In the wild type BR the amino acid at this position serves as a proton donor during the photocycle. The genetically modified BR-D96N, being deprived of an internal proton donor, depends on protons available in the medium. Thus the substitution permits to adjust the life time of the M state (cf. Fig. 9) from milliseconds in wild-type BR to minutes in BR-D96N [156]. A second 


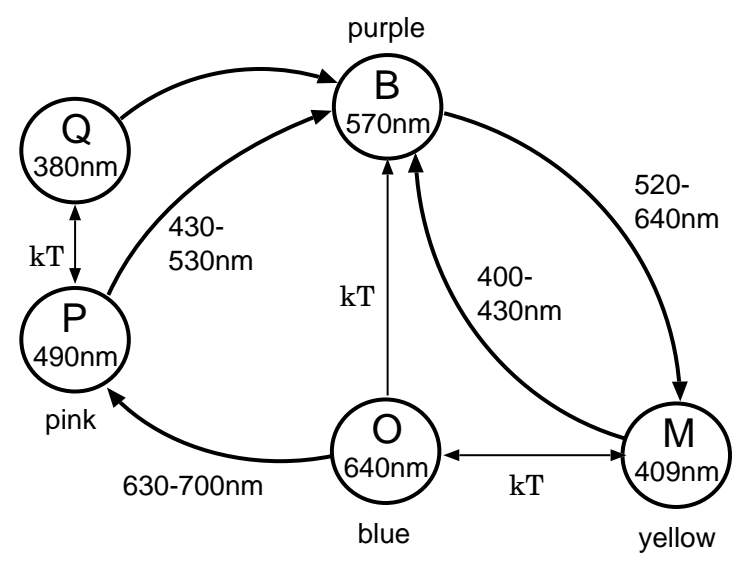

Figure 9: Transition scheme for BR states commonly used in information processing experiments. Circles represent spectroscopically identifiable states of the BR molecule. Characters in the circles show the (traditional) names of the states; the peak absorbance wavelength of each state is given below its name. A color name next to a state indicates the appearance of bulk BR in that state. Thermal transitions (kT) are shown with straight arrows, photonic transitions are drawn with bend arrows. The latter are labeled with the wavelength range suitable to induce the transition. States $\mathrm{P}$ and $\mathrm{Q}$ are not occupied under physiological conditions. The photoinduced $\mathrm{B} \leftrightarrow \mathrm{M}$ transition comprises a series of three intermediate states (not shown) and takes place in $\approx 50 \mu \mathrm{s}$. The M state facilitates the proton pumping. Under physiological conditions the thermal $\mathrm{M} \rightarrow \mathrm{O} \rightarrow \mathrm{B}$ transition will take place in a few ms. Several techniques have been developed to delay the $\mathrm{M} \rightarrow \mathrm{O}$ transition for short and long term storage. Note that the wavelengths can shift several $10 \mathrm{~nm}$ with changes in the physiochemical conditions of the BR film. Compiled from references $[155,156,157]$.

genetic variant, BR-D85N (the corresponding substitution at position 85) provides access to the non-physiological states $\mathrm{P}$ and $\mathrm{Q}$ (Fig. 9) under moderate conditions; in wild-type BR states $\mathrm{P}$ and $\mathrm{Q}$ are only available at low $\mathrm{pH}$ or through deionization [155].

Besides photochromic switching, BR exhibits a photoelectric effect [163]. Technical use of the latter is more challenging than of the former because it requires the preparation of films with highly oriented BR molecules. Nevertheless the possibility to interface BR electrically is the basis for several suggested applications. The use of BR in optical sensors for acquiring and low-level processing of the input signal $[164,165,166]$, and the BR-based optically reprogrammable artificial neural network design $[167,168]$ illustrated in Fig. 10, are examples. Photoelectric properties of BR have been altered with the introduction of chemical analogs of the wild-type chromophore into the BR protein [170]. This approach complements the genetic methods mentioned above to allow for further tuning of the protein to particular applications. An overview of the broad spectrum of BR variations obtained through either genetic or chemical modifications is offered by Vsevolodov [171].

The unique properties of BR may have potential in at least two domains of computing: high density data storage and optical processing. Several schemes for BR memories have been proposed (Hampp [155] offers a critical comparison). In general these schemes use the B state and $\mathrm{M}$ state of $\mathrm{BR}$ to represent the 0 and the 1 state of a data bit. Each logical bit is physically implemented by a large number of BR molecules and addressed by focusing a light beam on these molecules. The molecules can be embedded in a film or, for increased storage density, in a three-dimensional transparent matrix (e.g., polyacrylamide). Photonic switching of BR's conformational states in conjunction with optical state readout may be used to implement high-density memories [172]. In particular volume storage appears possible with simultaneous two-photon absorption (Fig. 11). It is not clear yet whether BR can compete with inorganic and synthetic storage materials, however, memory architectures that exploit BR's photoelectric properties or utilize a larger number of its conformation states could possibly give BR an advantage over conventional materials [155]. The use of BR in optical recording strips on identification cards is also under consideration [173]. 


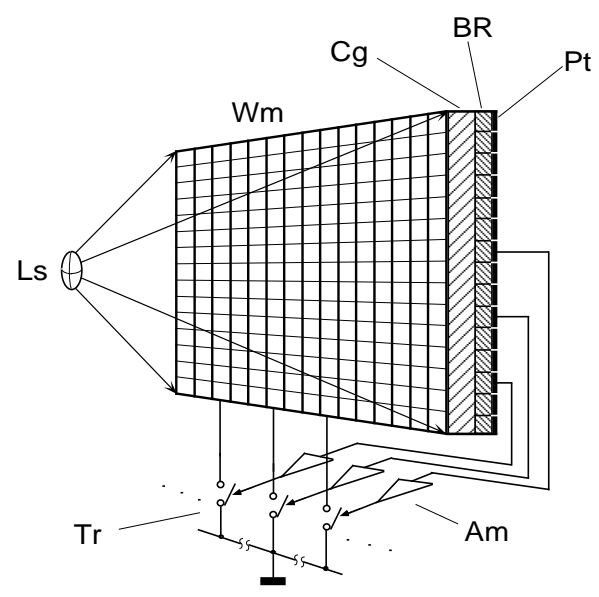

Figure 10: The hybrid opto-electronic neural network design suggested by Haronian and Lewis [167] exploits the photoelectric effect of bacteriorhodopsin (BR). Conventional electronics is used to implement the summation, amplification (Am) and thresholding (Tr) of the neurons. The interconnection strength of the Neurons is determined by the weight matrix $(\mathrm{Wm})$. Weights are represented by the state distribution of BR molecules in each matrix cell. A pattern of blue light from light source (Ls) can program the entire weight matrix in parallel. Flashing the conducting glass surface $(\mathrm{Cg})$ with yellow light yields in each matrix cell an electric potential corresponding to its programmed weight. Platinum electrodes $(\mathrm{Pt})$ at the back of the weight matrix $(\mathrm{Wm})$ feed the resulting photocurrent to amplifiers $(\mathrm{Am})$ that have been selected by the threshold switches (Tr). Finally a red flash restores the BR molecules that gave rise to a photocurrent. Reprinted from [169].

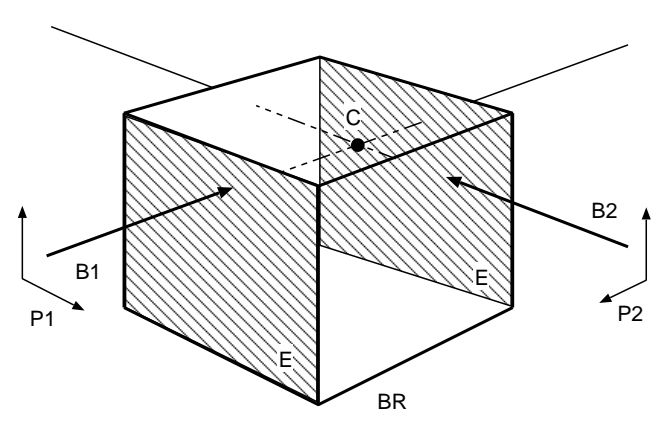

Figure 11: Three-dimensional high density bacteriorhodopsin memory based on the protein's nonlinear photochromic and photoelectric properties. A volume $\left(\right.$ several $\left.\mathrm{cm}^{3}\right)$ of transparent matrix material carries oriented molecules of bacteriorhodpsin (BR). Two laser beams (B1, B2) are spatially positioned in the orthogonal planes $\mathrm{P} 1$ and $\mathrm{P} 2$ to address a volume memory cell $(\mathrm{C})$ of $\approx 30 \mu \mathrm{m}^{3}$ at the beam intersection. Binary information is encoded as BR molecules predominantly occupying either the $\mathrm{B}$ or the $\mathrm{M}$ state. The choice of wavelength for the addressing beams determines what state is set in the addressed memory cell. Reading a memory cell is accomplished by setting the cell to the $\mathrm{M}$ state. If this operation is applied to a cell that was previously in the $\mathrm{B}$ state, the resulting $\mathrm{B} \rightarrow \mathrm{M}$ transition generates an electric potential between two electrodes (E) on the surface of the memory material. Thus the detection of the electric potential indicates that $\mathrm{B}$ was stored in the memory cell and the absence of the potential indicates that the state of the cell was M. If an electric potential occurred, the memory cell needs to be set to the B state to restore the information content that was present before the destructive reading operation. 
As mentioned above, light of a specific wavelength range can be used to change BR's conformational state and the conformation change is accompanied by a color change (i.e., a shift in the protein's absorption spectra) and hence can also be detected by optical means. Accordingly BR can be used as a photonic finite-state-automaton. The available state transitions (Fig. 9) are sufficiently rich to implement optical logical gates [174]. Another suggestion is the application of BR for edge enhancement in monochrome image processing [175]. In this approach a BR film is first illuminated with a spectrum chosen to prepare its molecules in a 1:1 ratio of $\mathrm{B}$ and $\mathrm{M}$ states. An object is illuminated with blue and yellow light which is then projected through a lens of high chromatic aberations onto the prepared BR film. The blue and yellow image of the object are approximately convolved with two different Gaussians by the lens. The interaction of the two convolved images with BR results in a state distribution of molecules in the B and the $\mathrm{M}$ state corresponding to the difference between these images. This difference map of two Gaussians convolved with the object image corresponds to an edge enhanced image of the object.

Considerably more powerful image processing operations have already been implemented. BR is particularly well suited to holographic applications because it supports not only absorption recording, but also polarization recording. The latter allows for separating the recorded signal from scattered light of the reference beam and thus yields a high $(45 \mathrm{~dB})$ signal/noise ratio when reading out the hologram [15]. Utilizing the holographic recording properties of BR Hampp and co-workers demonstrated an optical pattern recognition system based on a joint transform correlator $[161,155]$. The system receives two input images through electrically controlled spatial light modulators. One image may display a complex search space, whereas the other input image displays a pattern to be located in the search space. The light modulators are illuminated with coherent blue light. The light emerging from each light modulator is Fourier transformed by a lens and projected onto BR film. At locations in the Fourier plane where the input images have Fourier components in common, holograms can be formed in the BR film. Coherent green light is used for enabling the BR film to record these holograms and also serves to read out the BR film. The modulated green light emerging from the BR film passes through a lens, which implements the reverse Fourier-transform, before it is detected by a CCD camera that provides the electric output signal of the correlator. Bright spots in the camera image correspond to locations in the search space where the input pattern can be found. Furthermore, due to the excellent reversibility properties of $\mathrm{BR}$ the pattern recognition process can be repeated with the same BR film more than a million times [15]. This fact allows for the implementation of real-time holographic pattern matching at video frame rate $[161,15]$. The main limitation of such a system is apparently the low input bandwidth supported by currently available spatial light modulators [155].

If the input for the optical image processing system is provided by a real object, the above limitation does not apply. Juchem and Hampp built a lensless holographic image processor capable of utilizing the high resolution of BR films to some degree [157]. In realtime phaseshifting interferometry a measurement sensitivity of about $5 \mathrm{~nm}$ can be achieved. This system is commercially available since several years [176]. To the author's knowledge, BR, in form of the engineered variant BR-D96N, is the first active biomolecular component that is employed in a commercial information processing application [157]. Numerous patents have been filed with regard to BR-based information processing [156] and the biotechnology for producing BR on a commercial scale has been developed. It is likely that BR will establish itself as the first off-the-shelf biomolecular material for information processing.

\subsection{Excitable Chemical Media}

Bistable and astable chemical reaction systems have been considered as a potential substrate for computing. Generally the rate of a chemical reaction changes during the progress of the reaction due to the changing composition of the reaction medium. In some reaction schemes it is possible that the product of a reaction influences the rate of its own production through auto-catalysis or self-inhibition. As long as such a reaction scheme is far from its unique equilibrium state it may exhibit bistable behavior $[177,178]$.

Rössler discussed the implementation of a universal set of logic gates (cf. section 2) in homogeneous chemical media [24]. He suggests a bistable reaction scheme for the implementation of the NOT operation, and also to serve as triggering device for the realization of AND and OR operations by summing of the inputs and subsequent thresholding. In simulation studies it was 


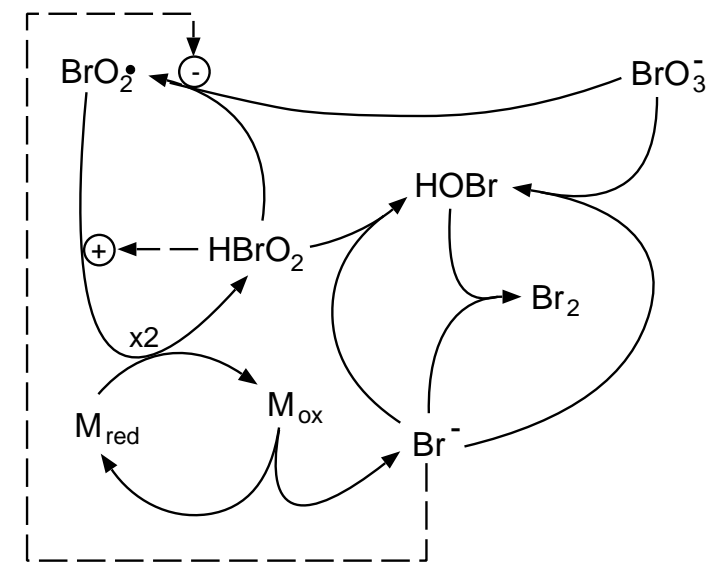

Figure 12: Core reactions in the oscillating Belousov-Zhabotinsky medium [177]. An autocatalytic cycle $\mathrm{BrO}_{2} \cdot / \mathrm{HBrO}_{2}$ leads to rapid increase in $\mathrm{HBrO}_{2}$ with a concomitant oxidation of a metal catalyst $\left(\mathrm{M}_{r e d} \rightarrow \mathrm{M}_{o x}\right)$. The resulting high concentration of the oxidized metal catalyst leads to slow formation of bromide ions $\left(\mathrm{Br}^{-}\right)$under regeneration of the catalyst in its reduced form $\left(\mathrm{M}_{\text {red }}\right)$. In sufficient concentration $\mathrm{Br}^{-}$ inhibits the autocatalytic formation of $\mathrm{HBrO}_{2}$ and consequently the further oxidation of the metal catalyst. The $\mathrm{Br}^{-}$ions are consumed by pathways leading to $\mathrm{HOBr}$ and $\mathrm{Br}_{2}$. When the $\mathrm{Br}^{-}$concentration drops below the level required for interrupting the autocatalytic production of $\mathrm{HBrO}_{2}$, a new phase of rapid oxidation of the catalyst begins. A second negative feedback (not shown) is based on organic free radicals [185]. For clarity only the interplay of major molecular species is shown here; for details see Ref. [186].

shown that networks of bistable kinetic devices can exhibit artificial neural network type learning behavior $[179,180]$ and thus the realization of chemical parallel computing architectures might be practical. Pettit designed an architecture for parallel chemical computing in which stacked reactor slices are interfaced through a pumped fluid [181], a design that has also been considered for biochemical processors [182].

That in fact the computational properties of excitable chemical media can actually be demonstrated in experiments was shown by Kuhnert et al. with a light-sensitive version of the BelousovZhabotinsky (BZ) reaction [183, 184]. Figure 12 illustrates the interplay of the main processes giving rise to the excitability of the BZ medium. A fast process with positive feedback gives rise to the slow build up of a substance that in sufficient concentration inhibits the fast process. When this negative feedback signal decays far enough, the fast process starts up again. By manipulating the negative feedback it is possible to couple the reaction to external stimuli.

A thin layer of BZ reaction medium [187], sensitized to light by means of a ruthenium complex as catalyst, is capable of performing elementary image processing steps such as contour enhancement and contrast inversion [184, 182, 188], and can be used for path planning [189]. The application of BZ medium in a thin unstrirred layer contained in a Petri dish allows for parallel optical input by projecting a light pattern on the surface of the reaction medium. Light intensity patterns are transformed by the catalyst into concentration patterns. The concentration patterns are then processed in parallel by the reaction-diffusion process in the pseudo-planar BZ medium. After a processing period that is typically in the order of seconds to several minutes, the pattern that developed is read from the surface of the reaction medium by a camera. Figure 13 illustrates an experimental setup for chemical image processing. A spatial resolution of approximately $2-5 \mathrm{lp} / \mathrm{mm}$ can be achieved $[190,189]$.

The BZ medium has only a limited flexibility for supporting different types of information processing operations. Currently two directions are pursued to overcome this limitation. First, hybrid computation schemes which combine chemical information processing with conventional computing steps are examined. Second, various methods for mechanically structuring the reaction medium are under investigation. Hybrid computing has been employed to find pathways in a maze [191] and to navigate around obstacles in a plane [194]. Shape recognition has also been addressed with a hybrid computing scheme $[195,196]$. In this case a reaction-diffusion medium 


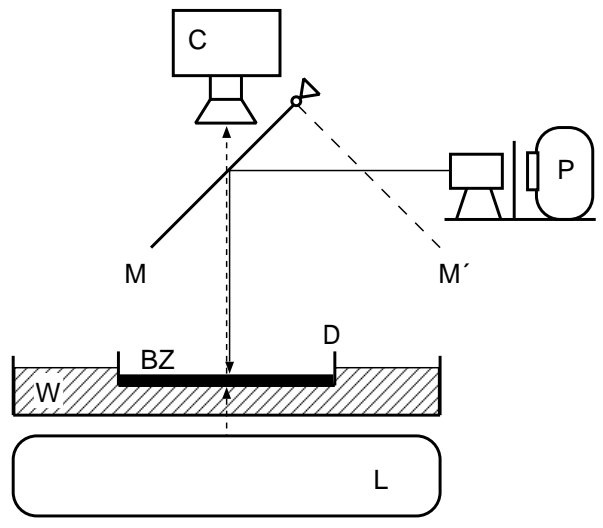

Figure 13: Typical experimental setup for information processing with a pseudo-planar light sensitive Belousov-Zhabotinsky rection medium. A Petri dish (D) with a diameter of $6-9 \mathrm{~cm}$ is filled to a height of about $1 \mathrm{~mm}$ with reaction medium (BZ) and held at constant temperature in a water bath $(\mathrm{W})$. Input images are projected with a projector $(\mathrm{P})$ and mirror $(\mathrm{M})$ on the surface of the $\mathrm{BZ}$ medium. After the reaction medium was exposed to the input image, the mirror is swiveled to position M'. The BZ medium is uniformly illuminated at low intensity with a light-box (L) and the progress and result of the chemical processing is recorded by a camera (C). Confer Refs. [191, 192, 193, 189] for variations.

based on $\mathrm{PdCl}_{2} / \mathrm{KI}$ was employed. Diffusion based processing on a macroscopic scale, however, is slow. For a Petri dish of $9 \mathrm{~cm}$ diameter a processing time of up to one hour may result if interactions among spatially distant points on the processing plane are required. Miniaturization will therefore play an important role for achieving a higher processing speed.

Spreading the reaction medium on a microstructured surface is another approach to increase the variety of implementable operations. For example the metal catalyst of the BZ medium (cf. Fig. 12) can be applied in a controlled pattern through ink-jet printing [197] or photolithography [198]. Imposing rigid spatial structures on the pseudo-planar BZ medium opened the path to a new range of chemical processors. Cellular automata with square and hexagonal grids of discrete BZ processors and defined coupling distances have been implemented [199]. Unidirectional wave propagation has been observed in macro [200] and micro [193] structures. Notable are in particular the chemical wave circuits of Steinbock, Kettunen and Showalter [197]. They used a metal complex that serves as catalyst in the BZ reaction in an ink-jet printer to print circuit networks with up to three processing stages onto a membrane. The membrane with the circuit was then brought into contact with a gel that contained a catalyst-free BZ reaction mixture. To operate the circuit the desired input lines on the membrane was touched with a silver wire. This triggers excitation waves representing the input pattern. The waves propagate through the circuitry and interact according to the geometry of the printed gates. The output of the circuit is represented by the comparative state of two output lines.

While it appears unlikely that chemical logic gates would ever become fast enough to compete with their electric counterparts, the realization of the gates mere indicates the possibilities of excitable media in inhomogenous spatial structures. Undoubtedly, harvesting the information processing power of such systems requires to adapt the computational concepts to their dynamics, rather than forcing them to conform to Boolean logic. Here also may be mentioned that there is considerable progress in the dynamic manipulation of reaction-diffusion media on the $\mu \mathrm{m}$ scale [201]. It is conceivable that in the future chemical processors could be miniaturized and implemented with on-chip technology [202, 203]. Miniaturization is of key importance for any device relying on diffusion for signal propagation. Asai et al. suggested an implementation of reaction-diffusion computing with a novel type of silicon semiconductor device using the diffusion of charge carriers [204]. While this suggestion abandons the chemical media altogether, it offers a conceptual architecture that is suitable for implementation with organic materials and is less brittle than conventional circuits with respect to variation in the device structure. The issues of diffusion speed and stochastic structures will be revisited in section 4 . 


\subsection{Self-assembly Computing}

Molecules are large enough to possess specific shape features, yet small enough to explore each other by diffusion. This fact together with short-range $(\approx 1 \mathrm{~nm})$ additive electrostatic forces endows molecules with the capability to spontaneously assemble into organized structures. A comparison of binding energies resulting from electrostatic interactions is shown in table 4. With molecular motions on a pico to nano second time scale the self-assembling macromolecules sample a wide range of interactions for a propitious free enthalpy [208].

If the shape of two molecules is complementary, they will be able to form numerous close contacts. The number of these contacts together with the proximity of the groups involved allows the potential energy to overcome entropy even at relatively high temperatures [209]. The interacting molecules will be held together in a well defined spatial arrangement by minimization of free energy. Furthermore, tuning the shape of the molecules modulates the strength of attractive electrostatic forces between them. Transient as well as durable supra-molecular structures can form through self-assembly. It almost goes without saying that the self-assembly of macromolecules is a mechanism with significant potential for the fabrication of spatially organized molecular systems, an aspect that will be discussed further in section 4.3.

Conrad pointed out that the jigsaw-puzzle like self-assembly of macromolecules can be regarded as a computational process and could be harvested to implement information processors [210, 211, 34]. He suggested a Gedankenmodell of self-assembly computing [35]. In this model, depicted in Fig. 14, input signals are encoded by molecular shapes. When brought together in a solution the molecules interact and self-assemble to a supra-molecular complex. A characteristic shape feature of the complex is detected by a receptor and amplified to provide the output signal. The key idea is the conversion of a symbolic pattern recognition problem into a free energy minimization process. Output "crystallizes" out of the input. The model abstracts intracellular information processing and in its description proteins were suggested as molecules for representing the input

Table 4: Binding energies for electrostatic interactions; compiled from [205, 206, 207]

\begin{tabular}{l|r|l}
\hline Interaction & Energy $[\mathrm{kJ} / \mathrm{mol}]$ & Comment \\
\hline Ionic & $600-1000$ & \\
Covalent bond & 360 & C-C bond in $\mathrm{C}_{2} \mathrm{H}_{6}$ \\
Lock-key fit & 88 & $\begin{array}{l}\text { biotin-streptavidin, } \\
\text { one of the strongest }\end{array}$ \\
Protein components & $40-80$ & combined effects \\
Hydrogen bond & $10-40$ & \\
Van der Waals & 3 & $\approx k T$ \\
\hline
\end{tabular}

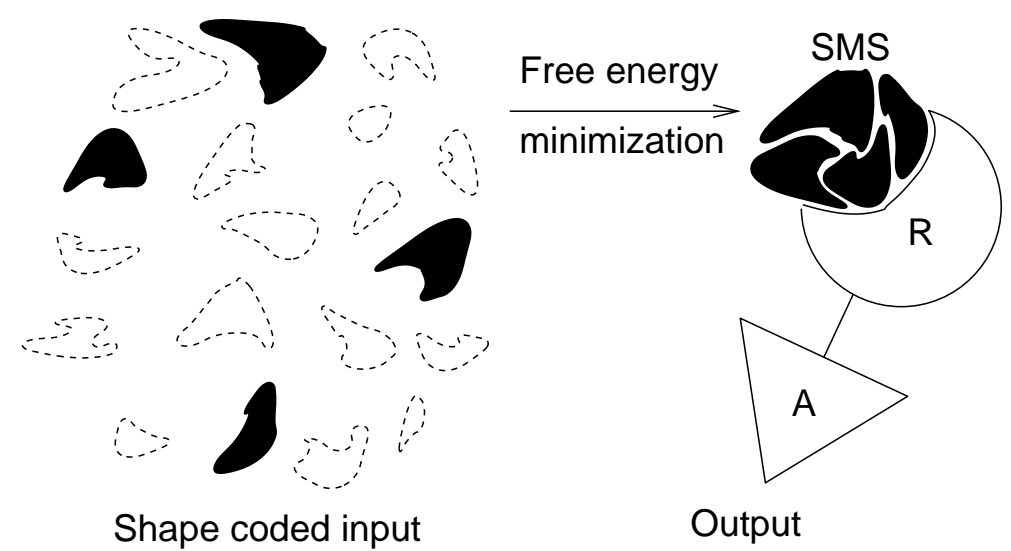

Figure 14: Abstract model of self-assembly processing. Symbolic input is represented by molecular shapes. These representations are driven by free-energy minimization to assemble into a supra-molecular structure (SMS). Characteristic shape features of the supramolecular assembly are recognized by a receptor $(\mathrm{R})$ and amplified $(\mathrm{A})$ to provide an output signal or action. After [212]. 


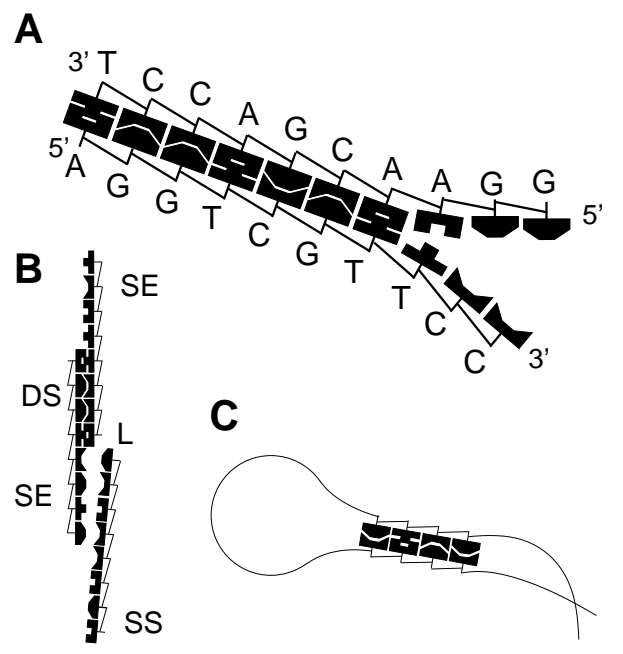

Figure 15: Hybridization of two complementary single stranded DNA oligonucleotides into a duplex DNA supra-molecule; panel A. Single DNA strands that are complementary only in segments can self-assemble to double strands (DS) with dangling single stranded segments, so called "sticky-ends" (SE). Further single strands (SS) or double strands with sticky ends can attach; panel B. Two strands that are bound in adjoint positions on a complementary strand can be enzymatically linked (L) with a covalent bond into a contiguous molecule. Intramolecular hybridization is possible if a sequence contains self-complimentary segments; panel C.

signals. Any of the three major groups of biomolecules, DNA, RNA and protein can be used for its implementation [213]. Protein can provide the largest variability in shape as well as the greatest flexibility in interactions. But along with this comes the fact that the self-assembly properties of proteins cannot be predicted form their sequences and consequently engineered protein-based self-assembly is not feasible. On the contrary DNA is much more restricted in its shape variations, but allows for relatively good estimation of the self-assembly products formed. RNA takes an intermediate position. On the one hand it shows much more structural variation than DNA, on the other hand the hydrogen bonding between the nucleotides of RNA can be more readily than the electrostatic interactions among amino acids in proteins. It may be noted that in the laboratory work with RNA is more cumbersome than with DNA, in part because enzymes that break down RNA are ubiquitous.

The relatively predictable self-assembly property of DNA molecules has found wide usage in molecular information processing schemes and is also of interest as self-assembling scaffolding material for novel fabrication methods (cf. section 4.3). Self-assembly of a short DNA oligonucleotide is illustrated in figure 15. DNA is a an unbranched polymer composed from a set of four building blocks that differ in a nitrogenous base. The four bases occurring in the building blocks are adenine, guanine, thymine and cytosine labeled in the figure A, G, T, and C, respectively.

Adenine and thymine can form two hydrogen-bonds and guanine and cytosine can form three such weak bonds [214]. Bases that can form bonds are said to be complementary. If the sequence of bases of an oligonucleotides is the complement of the sequence of another DNA strand, then numerous individually weak hydrogen bonds can form. In summation, these weak electrostatic forces lead to stable self-assembly complexes in form of a helical DNA double strand. The two single strands in such a DNA duplex can be separated by heat $\left(\approx 30-70^{\circ} \mathrm{C}\right.$, depending on the sequence) or by ionizing $\mathrm{pH}$ levels. This process is reversible, and DNA can be cycled through alternating assembly and disassembly stages [12].

As might be expected from the preceeding discussion, two DNA strands that do not perfectly match, i.e., do not have complementary bases at every position of their sequences may still establish a sufficient number of hydrogen bonds to stick together, albeit not as strongly as perfectly matched strands would. If numerous DNA strands interact, they will compete in the duplex formation. It is therefore necessary to calculate the binding strengths among all of them in oder to predict what structures will be formed and how the equilibrium of structures depends on factors like temperature, $\mathrm{pH}$ and salt concentrations. The thermodynamics of oligonucleotide 
duplex formation has been studied extensively by SantaLuica, Jr. [215, 216, 217], who also provided data for hybridization with dangling ends [218]. From the experimentally determined thermodynamic parameters the binding strength and secondary structure of DNA strands, and to a lesser degree also of RNA strands, can be predicted [219, 220].

The extraordinary density of information storage in DNA molecules became apparent during the time when the field of computer science was formed [214] and has been compared to existing technology [221]. A model for a molecular computer based on DNA was offered by Vaintsvaig and Liberman [21] in 1973. However, all the early considerations of DNA as a substrate for information storage or processing assumed that the sequence of bases on the DNA molecule would encode the information in a similar way as the base sequence encodes genetic information. Under this assumption every operation would require the breaking and forming of covalent bonds, a process that would presumably be slow. Moreover, an elaborate molecular machinery would be required to carry out the desired operations.

In 1994 Adleman published a paper [9] describing how to use the predictable self-assembly properties of a set of DNA molecules to solve a small instance of a problem that is in general intractable. His crucial insight was that specific sequences are not the only way of encoding information with DNA, short random DNA sequences (20 bases long oligonucleotides were actually employed) can be used as symbols to represent information. As a consequence operations to modify individual covalently linked bases in the sequences were not required. The short DNA molecules can self-assemble to larger structures based on hybridization (figure 15B) and the resulting structures can be detected with standard biochemical methods. In contrast to earlier experiments in molecular computing $[161,184]$ this work generated considerable interest within the computer science community and sparked a flood of theoretical papers [10] as well as a number of experimental studies on the potential of information processing with DNA. It is worthwhile to consider Adleman's elegant experiment in more detail as it was not only the first practical demonstration of molecular computing with DNA, but it also used a physical machine structure that is specific to a single instance of a class of problems. The latter concept carries over to other technologies.

Adleman devised a scheme for solving instances of the directed Hamiltonian path problem by exploiting the self-assembly properties of DNA molecules [9]. In this problem a directed graph with a designated start and a designated end node is given. The question to be answered is whether or not there exists a path in the graph that follows directed edges from the start node to the end node and fulfills the condition that every node of the graph is visited exactly once. No algorithm is known that would be able to answer this question for an arbitrary given graph with a method that is not essentially equivalent to trying out all possible pathways until either a valid path is found or no path remains to be checked. It is generally believed that for any problem in the fundamental class of intractable problems to which the Hamiltonian path problem belongs, i.e., the class of NP-complete problems, no better algorithm exists [222].

On a computer with a single processor an algorithm to decide whether a Hamiltonian path exists in a given graph would be implemented such that it tests one pathway after another. Taking heed of the fact that start node and end node are fixed, the number of possibilities for for different pathways in a graph can be calculated from the permutations of nodes on the path. Thus for a graph with $n$ nodes, there will be $(n-2)$ ! potential pathways. In case no Hamiltonian pathway exists, every possible path has to be checked until the negative answer is known. Accordingly the worst case time required to solve the problem on a computer that operates serially scales with the factorial of the number of nodes in the graph. Note, that the pathways can be tested independent of each other, hence, with several processor, the tests can be executed in parallel. In principle the checking of all pathways could be accomplished at the same time if one would have $(n-2)$ ! processors available. Thus the, with respect to node number, hyper-exponential requirement in time can be traded for an equivalent requirement in number of processors. This tradeoff would not help to overcome the intractability of the problem, but if the fine grained parallelism of interacting molecules can be put to work it could conceivably yield results for problems of a size difficult to solve with a serially operating computers.

Figure 16 illustrates the DNA computing scheme for solving the Hamiltonian path problem introduced by Adleman [9]. The graph solved by Adleman had seven nodes connected by fifteen edges. To simplify the description we consider the minimalist example of a four node graph. The directed graph for the problem instance is shown in panel $\mathrm{A}$. The node labeled $\mathrm{A}$ is designated as the staring node and the node labeled $\mathrm{D}$ is the end node. The question is whether a path 
A

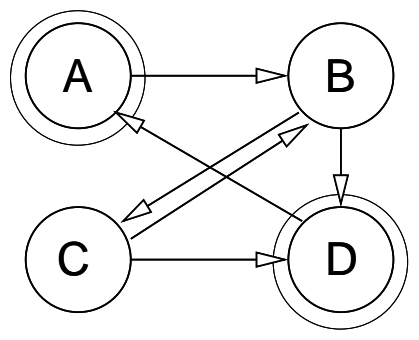

C
2: b' c
3: $c^{\prime}$ d d'
5: $c^{\prime} b$
4: b' d d'
$B:$ b $b^{\prime}$
6: d'a
c: $\bar{c} \bar{c}$

B

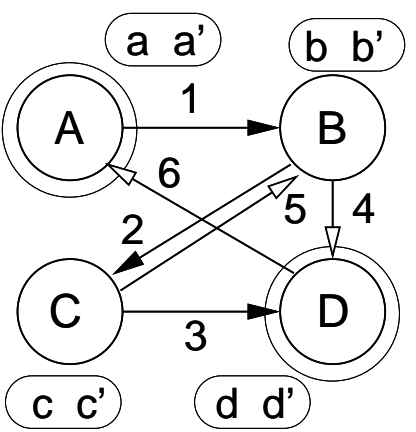

D

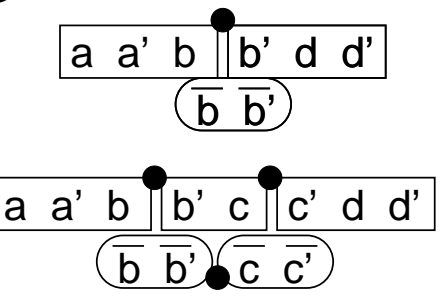

Figure 16: Solving an instance of the Hamiltonian path problem with DNA after [9]. Lower case letters indicate short oligonucleotides (10 bases). The sequence of these oligonucleotides is of no particular importance, as longs as the sequences designated by different letters differ significantly. A bar above a letter indicates a sequence complimentary to the sequence designated by the letter. See text for explanation.

exists from node $\mathrm{A}$ to node $\mathrm{D}$ visiting nodes $\mathrm{B}$ and $\mathrm{C}$ each exactly once. With four nodes there are $(4-2) !=2$ potential pathways to go from A to D passing through every node only once: $\mathrm{A}-\mathrm{B}-\mathrm{C}-\mathrm{D}$ and $\mathrm{A}-\mathrm{C}-\mathrm{B}-\mathrm{D}$. The former is a valid path in the directed graph and indicated in panel B with filled arrowheads. The latter, however, is not possible in the given graph, because there is no edge connecting node $\mathrm{A}$ to node $\mathrm{C}$. Of course there are other pathways from A to $\mathrm{D}$, for example, $\mathrm{A}-\mathrm{B}-\mathrm{D}$ or $\mathrm{A}-\mathrm{B}-\mathrm{C}-\mathrm{B}-\mathrm{D}$, but these do not fulfill the above stated condition for Hamiltonian paths. While in the given example only one solution exists, in general there may not be a unique solution; discovering any one of several solutions is then sufficient to decide the answer for the problem.

The aim is now to represent the graph shown in figure 16A with a set of DNA molecules in such a way that upon combining these molecules will form a structure that indicates whether a solution to this particular instance of the Hamiltonian path problem exists. To this end a 20 nucleotides long DNA molecule is formally assigned to each node in the graph. The actual sequence of the bases in these oligonucleotides is irrelevant as long as the sequences are orthogonal in the sense that each node has a sequence that does not interact with either the sequence of another node or the complement of a sequence of another node. Assigning random sequences to the nodes is likely to insure orthogonality in small graphs. The oligonucleotide sequence assigned to node $\mathrm{A}$ is designated in figure $16 \mathrm{~B}$ with $a a^{\prime}$, the one for $\mathrm{B}, \mathrm{C}$ and $\mathrm{D}$ are labeled $b b^{\prime}, c c^{\prime}$, and $d d^{\prime}$. The first character stands for the first 10 nucleotides in the sequence, the second, primed, character stands for nucleotide 11-20.

Based on the formal assignment of sequences to all nodes in the graph, a set of DNA molecules is synthesized (figure 16C). This set represents the entire graph and contains one DNA molecule for every edge and one molecule for every node in the graph, except the start and the end node. The DNA molecules representing nodes are simply the complements of the sequences formally assigned to the nodes, ie. $b \bar{b}^{\prime}$ and $c c^{\prime}$. A bar over a letter indicates the complement of the sequence designated by the letter. It is convenient to use edge 2 as example in describing the construction of DNA molecules representing edges, because edges leaving the start node or arriving at the end node (i.e., edges $1,3,4$ ) are treated somewhat differently. Edge two leaves from node B to which the sequence $b b^{\prime}$ was assigned. The first half of the oligonucleotide representing edge 2 will therefore correspond to $b^{\prime}$. The edge arrives at node $\mathrm{C}$ to which the sequence $c c^{\prime}$ was assigned. Accordingly the first half of the oligonucleotide representing edge 2 will correspond to $c$, thus 
an oligonucleotide with the sequence $b^{\prime} c$ will be used to represent edge 2 . The same method is applied to obtain the sequences for representing edge 5 and 6 . For reasons explained below, the edges 1,3 , and 4 are represented by longer sequences (30 nucleotides).

Edge 1 leaves from the start node and is therefore represented with a longer oligonucleotide. The first part of this molecule has the identical sequence to the one that was assigned to the start node $\left(a a^{\prime}\right)$ this is followed by the first half of the sequence formally assigned to the node where the edge arrives, i.e., by $b$, thus the full sequence representing edge 1 is $a a^{\prime} b$. The sequences for edge 3 and 4 are constructed analog to sequence 1, but ending with the full sequence assigned to the end node D. The full set of single stranded DNA molecules representing the graph shown in figure 16A is depicted in panel B of figure 16.

Now after DNA molecules for all sequences required for representing the graph have been synthesized, the molecules are combined and permitted to interact under conditions where ten complementary bases provide sufficient attraction to yield a stable self-assembly product. Due to the offset complementarity of the constructed molecules they will self assemble in the staggered fashion with overhanging sticky ends as illustrated in figure 14B. Two of the possible self-assembled structures are shown in figure 16D. The design of the sequences insures that an edges can only bind to nodes and vice versa. Every self-assembly product corresponds therefore to a path that exists in the graph. If the number of copies of each molecule in the set representing the graph is sufficient, then a self-assembly product for every possible non-cyclical pathway in the graph should be formed. If a Hamiltonian path exists in the graph then with high probability a self-assembly complex that represents this path has been formed.

The resulting self-assembly products are stabilized by enzymatic introduction of covalent bonds between oligonucleotides lined up next to each other (indicated with black dots in figure 16D). The self-assembled complex of molecules representing edges and molecules representing nodes are thus converted into two complementary DNA strands, one of which is the contiguous sequence of the molecules representing edges and the other is a continuous strand formed from the sequences representing nodes in the self-assembly complex. In the following steps only the DNA strand representing the edges is of interest.

Next, the reaction products are screened with analytic methods for the existence of a molecule representing a Hamiltonian path. Three selection steps are applied to eliminate sequences that do not meet the requirements for such a paths. First, a technique called polymerase chain reaction (PCR) is applied to increase the concentration of those sequences that contain the correct start and the correct end node. The replication is achieved by enzymatically synthesizing a DNA strand from individual nucleotides using the strand to be copied as a template for the synthesis [223]. The PCR process, illustrated in figure 17 requires two short chemically activated primer oligonucleotides that are chosen according to the two sequences representing the start node and the end node. On theoretical grounds the PCR amplification would not be necessary, because a later test applied to insure every node is present in the sequence will also select for the presence of the start and end nodes. However, the number of reaction products formed from the molecules representing a graph can be very large, indeed for a graph with loops there is in theory no limit on this number. Therefore in practice it is important to increase the concentration of those molecules that may represent a Hamiltonian path and PCR allows for the sequence specific selective amplification.

Subsequent to the PCR amplification of sequences with the correct start and end nodes, the sequences are separated according to their length by means of gel electrophoresis [223]. This process uses the fact that the mobility of DNA fragments in a gel is typically inversely proportional to the logarithm of their length [12]. In the PCR reaction illustrated in figure 17, for example, the second sequence shown in figure 16D $\left(a a^{\prime} b b^{\prime} d d^{\prime}\right)$ would also be amplified exponentially. From the length of the oligonucleotides used to encode an edge together with the number of nodes in the graph and the fact that every node has to appear exactly once in a Hamiltonian path, the exact length of any molecule that could represent a valid path can be calculated. It is here where the above described special representation of the edges originating in the start node and the edges terminating in the end node comes into play.

If sequences with the correct length have been detected it is now necessary to verify that every node in the graph is actually present in the path represented by at least one of the sequences. While not possible in the example graph (figure 16A), in general a loop in the graph could lead to the formation of a DNA molecule representing the correct start node and end node and having correct length, but with a node that occurs more than once in the path and correspondingly one 


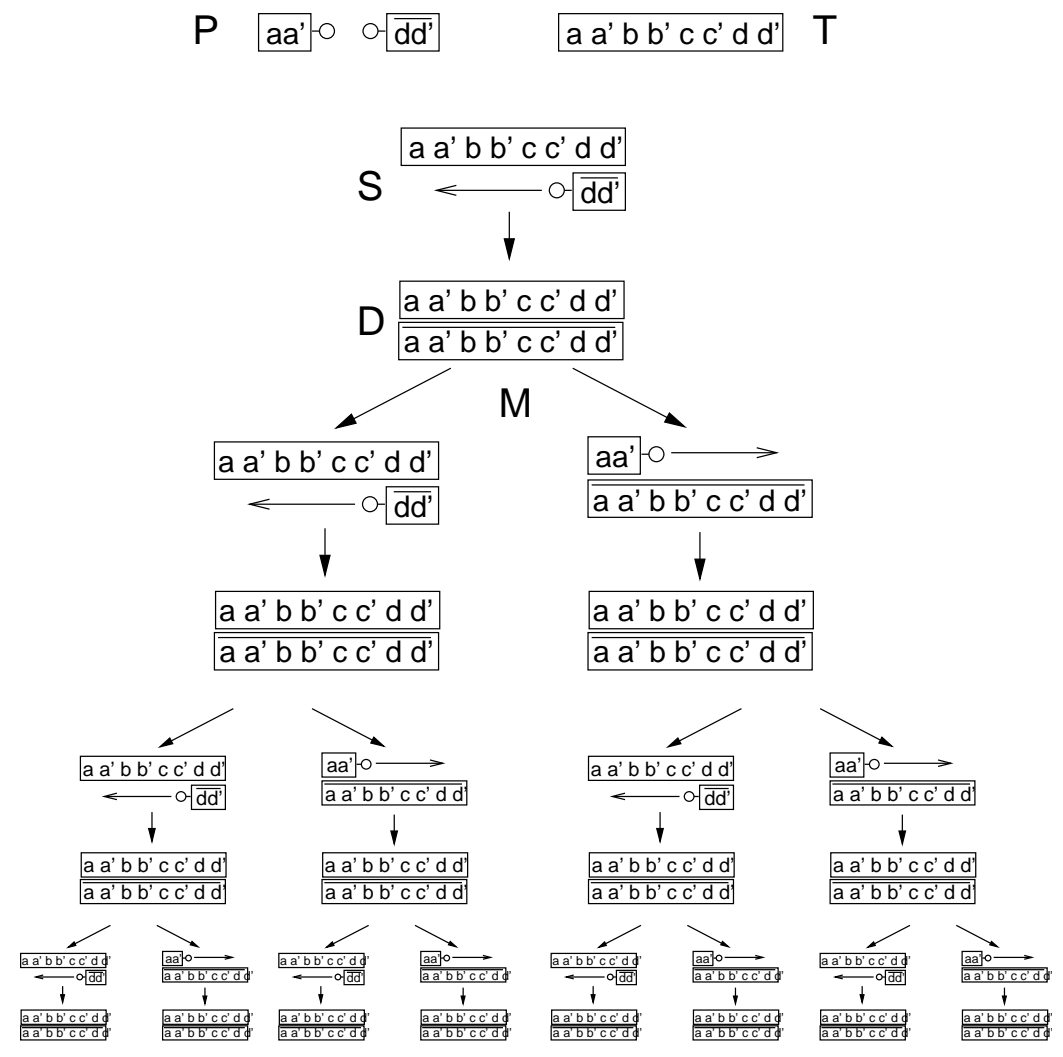

Figure 17: Polymerase chain reaction exponentially increases the concentration of a specific target sequence $(\mathrm{T})$. Two primers oligonucleotides $(\mathrm{P})$, one equivalent to the complement of the end, the other one equivalent to the start of the target sequence are provided. The complementary primer binds to the target strand and enables the polymerase enzyme to synthesize (S) a complementary copy using the target strand as template. The resulting duplex (D) is melted by heating (M) into single strands. Synthesis can now proceed on both strands, using both primers. This cycle is repeated several times. By using a polymerase from a thermophilic bacterium that withstands the melting phase and supplying sufficient nucleotides, the process only requires cycling of the temperature. Strands that match only one primer are amplified linearly with the cycle number, strands that match start and end primer are amplified exponentially.

or several nodes missing from the path. The testing for the presence of the nodes is done one node at a time.

The sequences remaining after the gel electrophoretic selection for length are tested for the presence of a given node with hybridization probes. Molecules with the sequence representing the node of interest in the set of molecules that represent the graph (figure 16C) are chemically coupled to a solid support. The mixture of sequences to be tested is brought into contact with the solid support and sequences that contain the representation of the node will bind to the support. All sequences that did not bind to the support are washed away and discarded. Then the bound sequences are released from the support, e.g., through warming. Any sequence in this remaining set is now known to contain the representation of the node tested for.

The above procedure is repeated with the next node using he sequences remaining after the previous test until the test has been executed for every node in the graph. If DNA molecules are remaining after the final test then a Hamiltonian path exist in the graph and conversely if no DNA molecules remain there exists no such path. Note, that the testing for the presence of a node in the sequence is the only operation in this computing scheme for which the number of executions depends on the size of the graph. The number of required testing steps increases linearly with the number of nodes [9].

The linear scaling of effort with regard to graph size as compared to the factorial scaling for a serial algorithm attracted attention. Clearly, trading space for time does not overcome the intractability of NP-complete problems. But would it be possible push the boundary of what 
can be computed in practice? The answer to this question would have immediate application in the assumptions made regarding the security of cryptographic systems.

Now, at the end of its first decade the field of DNA computing, however, appears unlikely to advance the limits of practical computation. Three factors are impeding the realization of many theoretical suggestions that have been published in the past decade:

1. DNA molecules are macromolecules [224]: While it is common to handle mol quantities $\left(1 \mathrm{~mol}=6.0232 \cdot 10^{2} 3\right.$ particles $)$ of small molecules, the same can be impractical for macromolecules. A mol of water fits in a brandy glass, but a mol of E. coli DNA weights 2.4 tons; dry. (The mean molecular weight of a nucleotide is $\approx 308$.)

2. Hybridization is not string matching [225, 226]: The interaction of DNA strands cannot be described well with in logic formalism, physics has to be taken into account. It is possible to calculate from empirical parameters good predictions for the association of DNA oligomers [216], but it is not easy to design a large set of oligomers free from unintended interaction. In addition to the variation in thermodynamic stability of assembled sequences, DNA exhibits considerable secondary structure variation [227, 228]. For computing concepts that rely on high-fidelity logic operations the variability in the behavior of oligonucleotides is problematic.

3. Diffusion does not scale up [229]: Diffusion is fast on microscopic scale, but very slow on macroscopic scale (cf. section 4.1). Mixing is also difficult, because the highly asymmetric DNA molecules break easily.

In combination point one and three place a limitation on how far the time-space tradeoff for complex problems can be taken. Massive parallelism requires DNA molecules in large numbers, which in turn requires a large volume and in the large volume the speed of interaction among molecules is low. At present there is no technique in sight that would be suitable for scaling DNA computing to a level relevant for the practical solution of complex problems. Nevertheless, an application in the field self-organizing materials appears plausible and is discussed in section 4.3.

The conformational dynamics of polynucleotides that interferes with attempts to realize formal string processing concepts in form of what has been termed [226] "hybridization logic", however, can conceivably be used for information processing in a non-programmable device [230]. If conformational dynamics is employed rather than suppressed then biomacromolecules exhibiting a more pronounced variability in their secondary and tertiary structure than what is observed in DNA become of interest for implementations [213]. Conformational switching in the BR protein, discussed above (section 3.3), is at the core of its information processing applications. The following section describes more generally computing concepts based on the intra-molecular dynamics of macromolecules.

\subsection{Conformation-based Computation}

The conformation of a molecule describes the preferred spatial position of its atoms. Transitions among different conformations of a molecule do not require breaking of covalent chemical bonds. The conformation of macromolecules has a strong influence on its physical and chemical properties.

Proteins, i.e., amino-acids polymers, show a particularly pronounced variability in stable conformations found within the same class of molecules. The conformational variability among ribonucleotide polymers, for example, is significantly lower [231]. This variability of protein spatial structures affords large flexibility in function and underlies proteins' central role in mediating almost all functions in living cells. The present section will therefore focus on information processing with protein conformation. However, all macromolecules show conformational change and conformation-based computation can be implemented with molecules other than proteins; the next section will describe an implementation with RNA.

Rotation around the covalent bonds that hold the amino acid chain of a protein together allows for extensive flexibility in the chain. As a result of electrostatic interactions among the amino acids and between the amino acids and their chemical surround (e.g., water, and salts or other proteins [207]) these flexible chains can curl up into compact three-dimensional shapes. During this folding process amino acids distant from one another in the one-dimensional sequence interact through their proximity in space [232]. The cumulative effect of numerous interactions 
that are individually weak relative to thermal energy lead in this way from a sequence to an elaborate spatial gestalt. Experiments with random sequences ranging from 80 to 141 amino acids in length indicate that, even under the restriction to physiological conditions, many of the large set of possible sequences are likely to fold [233, 234, 235]. Folded proteins in aqueous solution expose hydrophilic amino acids at their surface and bury hydrophobic amino acids in their core. Within cells folding into the functional form occurs spontaneously for some proteins while others require the assistance of special proteins, so called, chaperons [236]. The need for chaperons arises in part from the high density of nascent proteins locally present in the cell during protein synthesis. Chaperon bind to partially folded proteins with exposed hydrophobic groups to prevent their aggregation [237]. The view that an amino acid sequence will fold into a unique native structure was held for a long time [238], but evidence that seems to indicate that variation in the physicochemical milieu in which a protein folds may result in folding variants supporting alternate functional capabilities is accumulating [239]. The folding equilibrium of a protein can be influenced, for example, by direct interaction of the solute molecules with the protein [240] or indirectly by solutes that alter free energy of bulk water and thus affect the strength of hydrophobicity [241].

The significance of the folding process as a physical rather than a formal mapping from sequence to function has been stressed repeatedly by Conrad [242], especially with regard to evolvability $[243,244,245]$ and the role of proteins in molecular computing [29, 246, 212]. It is this selforganizing step that fundamentally differentiates the building block principle as employed by nature from the planned assembly of modular components commonly used by human engineers. Arguably the latter is precluded by the difficulty of mapping between spatial structure and amino acid sequence $[247,248]$. The folding itself is controlled by kinetic as well as thermodynamic factors. It has been suggested that energetically different metastable folding states with similar tertiary structure are assumed under the kinetic influence of the physiochemical context during the folding [249]. Force-induced unfolding of single molecules [250, 251] may in the future shine light on the variation in characteristics among the folding products of identical amino acid sequences.

The spatial shape resulting from the folding is stabilized by intramolecular forces and proteinsolvent interaction $[252,253]$. Still, the folding process does not simply terminate at an arbitrary point in time [254]. The folded shape possesses a defined agility that enables it to assume numerous conformational states $[255,256]$. Cavities in the folded protein allow for the amino acid side-chains to rotation required for conformational change [257]. The energy of the conformational states and nearly isoenergetic substates can be described by a hierarchy of rugged energy landscapes [258, 259]. Under given milieu conditions a distinct subset of these states is favored. A change in physiochemical context can induce a switch to a different favored set of states [260, 261]. In fact the conformation of a folded protein remains so flexible that Gavish likens hydrated proteins to highly associated viscous liquids [262].

The spatial structure of the folded state and its associated concerted conformational motions are crucial to the function of a protein. That the origin of the highly specific function of enzymes, i.e., catalytically active proteins, lies in their spatial structure was proposed by Thierfelder and Fischer and led Fisher [263] to his well known metaphor: for the enzyme to have an effect it has to fit to the substrate like a lock to a key. Since then the picture of enzyme-substrate interaction has become increasingly more dynamic [264, 265] and it is now thought that the above noted selection of conformational substates is also involved in enzymatic catalysis [266]. It may be noted that here again an instructive paradigm [267] is shifting towards a selective paradigm $[268,269]$, a situation that is common in biological research, as Jerne [270] observed.

Motions of the protein occur during catalysis from substrate binding, over transition state binding to product release [271]. The fast low energy conformational changes can be grouped into hinge motions and shear motions [272]. The former are mainly constrained by secondary structure and result in a few amino acids undergoing large motions, while the latter are also constraint by tertiary structure and involve many amino acids along the shearing interface undergoing small motions. A rather low catalytic activity of rigid antibodies for transition state analogs and other static low molecular weight enzyme mimics point towards the importance of conformational dynamics for the efficiency of enzymes [273, 274]. Intriguingly, the conformational state of a protein may depend on its previous interactions [275]. Observations on single molecules of the enzyme horseradish peroxidase, for example, have shown that conformational states are affected by past states over a range of seconds [276]. 
From the viewpoint of technical applications it is interesting to note that conformational motions in proteins have been recorded from nanosecond to second time scale by fluorescence spectroscopic methods (e.g, $[277,278]$ ) and conformational change induced by alterations of the physiochemical context has also been observed via fluorescence (e.g., [279]).

The dynamics of proteins facilitates the remarkable specificity and speed of enzymatic catalysis. It is also the key to precise and versatile regulation of protein mediated catalytic activity. For the signaling nodes connecting multiple pathways in a cellular regulation network two regulatory effects are found [280]. In the first, cooperative regulation, one signaling substance enhances the sensitivity of the enzyme for another signal without affecting the maximum response level of the enzyme. The second is multi-state regulation, in which the signals modulate the output level, but do not alter the sensitivity of the enzyme to signaling substances.

The effect of regulator substances on binding sites is transmitted by conformational motions to the catalytically active site [32, 281, 282]. These motions may include high-energy transitions with repacking [283] and may spread rapidly by direct conformational interactions among proteins that are in contact $[284,285]$. The recognition of the conformational state of one protein by another protein plays a role in regulation as well as intracellular quality control [286].

With the above description of protein conformational dynamics in mind it is evident that a protein is not a simple molecular switch but rather a complex nonlinear processing network of thousands of nuclei and tenth of thousands of electrons coupled in a highly refined pattern of electrostatic interactions. This network does not only comprise the protein itself, but also molecules bound to it, and furthermore is also coupled to the detailed structure of its solvent [264]. In general the solvent is water, but for transmembrane proteins the structure of the lipid bilayer hosting the protein is also relevant. Accordingly proteins can sense and react to alterations in the solvent structure caused by molecules that are not in direct contact with them [241]. The fusion of sensory information in a seed's decision to germinate can serve as an analogy to underline the contrast between a molecular switch and the conformation-based information processing of a protein reacting to the physiochemical context of surrounding milieu features [287]. The conditions under which a seed germinates and also the conditions under which an enzyme acts do not have simple logic descriptions. Numerous factors from an essentially open context interact in a time dependent fashion to yield a probabilistic response. Only under narrowly prescribed conditions that largely fix the potential signal inputs to the protein is a description of the protein behavior as logic gates appropriate [288, 289, 290]. Even simplistic descriptions of enzyme kinetics, however, are equivalent to quite sophisticated circuits [291] and in general a view of individual proteins as black-box computers [292] is more apt.

The elaborate intramolecular dynamics of proteins, commonly regarded as a nuisance for protein structure prediction, is a resource that can usefully be employed for information processing [293, 294, 295, 38]. The concept of conformation-based computing is illustrated in figure 18. A macromolecule, for example a protein, is perceptive to the physiochemical milieu in which it is immersed. Intricate microphysical interactions (described above) between the protein and the milieu enable the protein to selectively respond to specific signal patterns in the milieu while ignoring other features in its complex physiochemical surroundings. Thousands of atoms comprising the protein act as a network to concertedly fuse the information carried by the milieu signals. The effect of the milieu on the protein is a bias in the probabilities for the protein to occupy a particular subset of conformational states. Different conformational states are associated with differences in function. Accordingly the output could be a chemical signal, if the protein is an enzyme, it could be a shift in its absorption spectrum (as in the BR molecule discussed in section 3.3), or a fluorophore and quencher could be attached to the protein to obtain information about its conformational state (cf. sections 3.2 and 3.7). The number of potential milieu factors that could conceivably be used to encode input signals is virtually boundless [296] as there is no need to limit the selection to chemicals and concentrations found under physiological conditions.

Biological cells contain thousands of proteins. In unicellular organisms, such as amoeba or paramecium, all the information processing is mediated by molecular networks. Chemical signals from the environment are communicated through the cell membrane, either directly or by triggering the release of internal chemicals through interaction with membrane receptors. The signals influence specific proteins in these networks, leading to cascades of reactions that eventually culminate in the action of the cell. In an artificial device the output could, for example, be mediated by fluorescence probes attached to the protein. But if the protein is an enzyme, changes in physiochemical context that alter the preferred conformational state of the enzyme 


\section{Milieu signals}

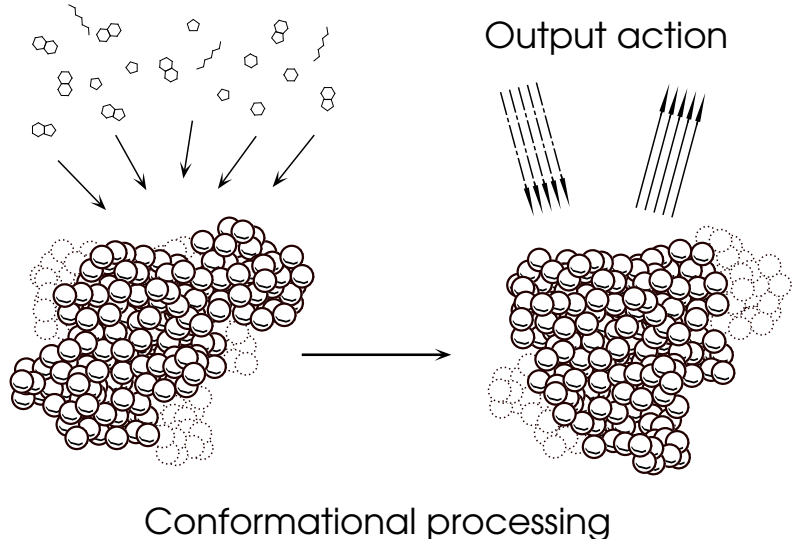

Figure 18: Conformation-based computing. Chemically coded input signals impinge on the conformational dynamics of a macro molecule. The complex conformational dynamics provided by a network of thousands of electrostatically coupled atoms affords rich nonlinear factor interactions among the input signals. Alterations in the macromolecules conformational state distribution yield the output signal, either by modulating chemical or physical properties of the macromolecule. Reprinted from [38].

most often will alter the activity of the enzyme and hence the speed of the reaction catalyzed by the enzyme. Therefore the catalytic activity of such enzymes provides a sensitive indicator of conformation change.

Experimental studies with the enzyme malate dehydrogenase (MDH) showed that divalent ions can be used to modulate its activity [297]. If inputs are coded with ionic signals and the concentration of the product formed by the reaction catalyzed by $\mathrm{MDH}$ is regarded as the output signal a variety of logic gates (AND, OR, XOR, NAND, NOR) with a response time of less than $10 \mathrm{~s}$ have been implemented using $\mathrm{Mg}^{2+}$ and $\mathrm{Ca}^{2+}$ as input signals [298]. Figure 19 shows ion concentrations for signal encodings that yield logic OR, XOR or NAND output. Recall that the NAND operation is a universal operation in the sense that any other logic gate can be composed from a network of NAND gates (cf. table 3). Of particular interest is the XOR operation as the simplest not linearly separable function. For implementing the XOR operation a nonmonotonic response of an elementary component is required since no non-monotonic operation can be implemented with a network composed of only monotonic elements [299].

The non-monotonic MDH response is likely due to opposition between an activating effect and a suppressive effect. Ions not involved as reactants have in numerous cases been found to enhance enzymatic activity [300, 301] and an activating effect has been found [302] for $\mathrm{Mg}^{2+}$ (and exists according to our observations [297] also of $\mathrm{Ca}^{2+}$ ) affecting $\mathrm{MDH}$. On the other hand, high ionic strength unspecifically suppresses MDH activity [303]. For the reverse reaction a non-monotonic effect on MDH activity has been observed in response to phosphate ions [304] and recently it was reported that several other ions can also be used to achieve non-monotonic modulation of $\mathrm{MDH}$ activity [305]. In this study the enhanced activity of $\mathrm{MDH}$ in the presence of ions at low concentration is attributed to a stabilization of the dimeric MDH supramolecular complex, while the reduced activity at increased ionic strength is attributed to conformational change in the enzyme.

In the experiments with MDH chemical signals were used to influence its conformation. It is also possible to alter the conformational state of a molecule through optical signals. The conformational state switching of BR described in section 3.3 is a case in point. Sivan and Lotan investigated the potential of photoinduced conformational change for enzymatic computing [306]. They use two light activated components in their system. First, a chemically modified version of the enzyme $\alpha$-chymotrypsin the catalytic activity of which can be controlled by radiation with UV light. And, second, the $\alpha$-chymotrypsin inhibitor proflavin. Proflavin can be reduced photochemically with blue light to acridan which does not inhibit $\alpha$-chymotrypsin. With this combination of two reversibly photo controlled components the authors demonstrated an enzymatic AND gate with optical input [307]. The output signal is, as in the MDH system, thresholded enzymatic 


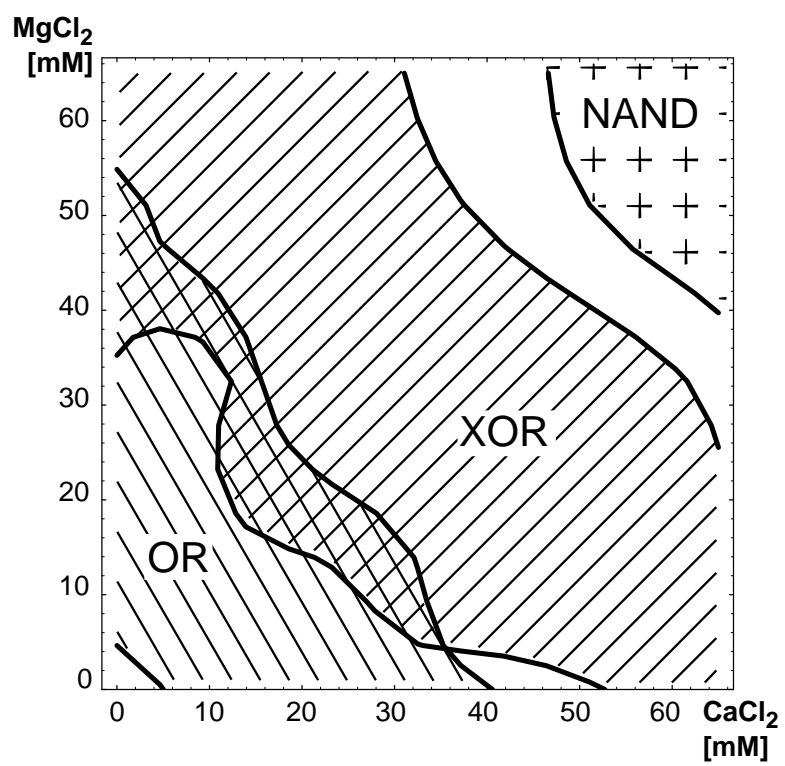

Figure 19: Logic operations from thresholding the response of porcine mitochondrial malate dehydrogenase to signals coded by a mixtures of $\mathrm{MgCl}_{2}$ and $\mathrm{CaCl}_{2}$. A point in the plain corresponds to the ion concentrations in a buffer solution used for representing a 1-input signal. The 0-input signal is represented by the buffer solution without $\mathrm{Mg}^{2+}$ and $\mathrm{Ca}^{2+}$. For the concentration zone where both OR and XOR functionality are possible, the choice of threshold level determines the operation. Reprinted from [297].

activity. A 1-signal is produced only if the enzyme is in its active conformation and the inhibitor is in its inactive conformation. A technical difficulty with the reversible operation of this gate is the need to change of oxidation state of proflavin. In the reported experiments aeration and deaeration was used in addition to irradiation with blue light to facilitate the state change of the proflavin [307].

Protein conformational change in its most extreme form leads denaturation of the protein's tertiary and secondary structure and results in a random-coil form of the poly-amino-acid chain. If the denaturing agent is removed, many proteins will refold to their native conformation [12]. A substance commonly used to unfold proteins is urea which disrupts the noncovalent interactions among amino acids. The electron-transferring protein cytochrome $c$ contains among its about 100 amino acids a single tryptophan. Tryptophan is a fluorophore, but in the native conformation of cytochrome $c$ the tryptophan's proximity to the heme group of the cytochrome results in efficient quenching of the fluorescence. Unfolding of the protein separates the heme group from the tryptophan and consequently the tryptophan fluorescence can be detected. This denaturation dependent fluorescence of cytochrome $c$ has been used to implement an AND and an OR logic gate [308]. In the same work an XOR gate was also reported, but it is trivially based on the neutralization of equal amounts of acid and base used as input signals. An interesting aspect of the implementation of the cytochrome $c$ gates is the use of a dialysis membrane to allow for continues signal input, yet the response time $(\approx 20 \mathrm{~min})$ of such a setup is slow.

It is important to emphasize that even though the preceeding discussion is phrased in the language of logic gates, the modulating enzymatic activity with conformation-altering signals results only in the most simplest cases in gate like behavior. The context dependence of $\mathrm{MDH}$ activity illustrates the complexity of conformational signal processing and, the point made above, that a comparison with logic gates underestimates the computational capabilities of enzymes. The enzyme $\mathrm{MDH}$ is under investigation for almost 70 years [309]. Considerable effort has been invested in elucidating the various factor interactions associated with the kinetics of its catalytic activity $[310,311,312,313,314,315,316]$. Some thirty years ago it was found that the complexity that had emerged from the study of this one protein far exceeded expectations [317]. The investigations continued $[318,319,320]$ and new effects are still discovered even within the limited range of compounds known to be of physiological significance [321, 322, 305]. 

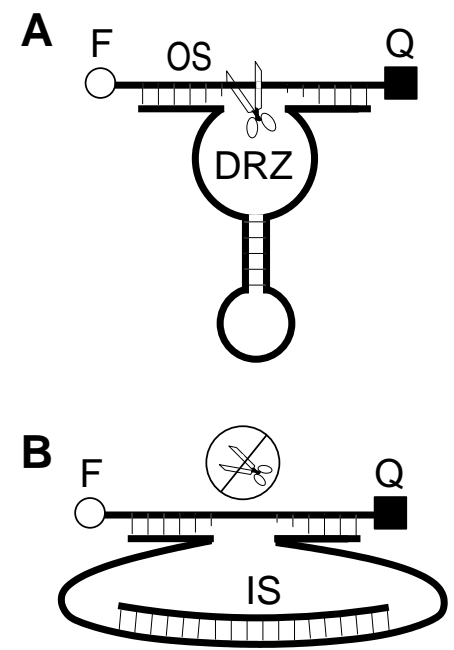

Figure 20: Deoxyribozyme NOT gate [325]. In the absence of an RNA input strand (IS) the deoxyribozyme (DRZ) is active and cleaves the output strand (OS). Upon separation of the fluorophore $(\mathrm{F})$ and quencher $(\mathrm{Q})$, both of which are connected connected by the intact output strand, a fluorescence signal indicates a 1-output for the gate. Moreover the two separated parts of the output strand can serve as input for further gates; panel A. If a the input strand (IS) is present, it will self-assemble with the deoxyribozyme and distort its conformation. The deoxyribozyme is no longer active, the output strand is not cleaved and consequently no output signal is produced; panel B.

The complex context-sensitivity of macromolecular conformational dynamics poses a challenging task for investigators studying the physiological function of these molecules. But it is also a computational resource for living cells. It enables the implementation of molecular control networks that fuse signals with far fewer components than what would be required if simple, context-free switching nodes would be employed. The chemotactic behavior of bacteria is a case in point $[323,324]$.

We will now turn to a recently introduced computing concept that combines conformational computation with elements of molecular switching and self-assembly computation.

\subsection{Allosterically Regulated Deoxyribozymes}

In a fascinating series of papers $[325,326,327]$ Stojanovic and Stefanovic first described the development of a universal set of nucleic acids-based logic gates with the crucial property of using RNA molecules for both input and output signals. These logic gates are DNA-based enzymes, called deoxyribozymes (DRZ), that cleave specific RNA oligonucleotides. Their enzymatic activity can be altered by oligonucleotides capable of binding to the DRZ and thus altering the conformation of the DRZ. This process is illustrated for a NOT gate in figure 20. Input and output use the same type of signals, i.e, RNA oligonucleotides, and, moreover, these signals can be specific due to their distinct nucleotide sequences. Second, the flexibility of allosterically controlled deoxyribozymes has then impressively been demonstrated by implementing a simplified tic-tac-toe game [327]. The network, effectively a molecular automata, responds according to fixed rules to up to 8 distinct input molecules with up to 8 sequential output signals. The rule set is implemented with a permanently active DRZ and 23 DRZ logic gates, 19 of which have three inputs $(\mathrm{f}(\mathrm{x}, \mathrm{y}, \mathrm{z})=\mathrm{x}$ AND y AND NOT $\mathrm{z})$. In theory the aforementioned properties of DRZ should allow for the implementation of networks of logic gates. This would require that one part of a cleaved output strand can act as input for a subsequent gate. However, the logic operations demonstrated so far have been limited to parallel operation of gates [326, 327] and sequential operations have not yet been shown.

We must not omit to mention here that the DRZ gates have slow switching times especially under physiological conditions. It may take 15 to 30 minutes to establish a good signal to noise ratio for the output, the stable output being maintained in vitro for several hours [327, 326]. 
Under non-physiological conditions switching times of less than one minute have been reported for one gate [326].

Simple logic circuits operating at time scales of minutes are exceedingly slow in comparison to their solid-state counterparts and provide no path to advancing computing power. Nevertheless, such circuits may find broad application as they can potentially be integrated into living cells. The implementation of artificial control structures in into cells would allow for dynamic control over cellular metabolic pathways [328] and gene expression networks. It is in this context that switches [8] and logic gates in genetic networks [329] are studied.

That in vivo control of cells for medical and environmental applications will become an application niche for molecular computing had been recognized a decade ago [330, 225]. Recently an in vitro study suggested how artificial intracellular control may be used in cancer treatment [331]. A stochastic molecular automaton recognizes RNA strands, the presence or absence of which in combination indicates an aberrant cell. If the molecular automaton reaches a state that indicates a positive diagnosis, the cell will be treated with the release of an oligonucleotide strand. This strand is chosen to interfere with the synthesis of a particular protein by binding to the messenger RNA that encodes for this protein.

The following section departs from the slow information processing adequate for controlling molecular processes such as gene expression or the cell cycle, and describes an application of molecules aimed at computations infeasible with conventional architectures.

\subsection{NMR Quantum Computing}

Molecules play the central role in a computation scheme quite different from the previously discussed concepts. The the two states of an input bit of a classical logic gate can be represented by eigenstates of a quantum mechanical system. Appropriate physical manipulation of the system can then move the system to a new state in which the output of the gate can be obtained from determining the state of a particular component of the quantum mechanical system. The fact that in a quantum system bits do not need to be in one of the two states but can also be in superposition of both is of particular interest and such bits are termed "qubits" to indicate this property. Quantum logic gates can operate on qubits in superposition state and yield a superposition of the output for both eigenstates of the qubit in one operation [332]. In principle this quantum parallelism extends to quantum computers assembled from quantum logic gates. A quantum computer with $n$ input bits could thus map simultaneously all possible $2^{n}$ inputs into a superposition output state involving the results of each of the possible inputs. While it is not feasible to retrieve from the output state the result for each individual input, global properties of the output may be useful for particular problems. Global properties could be obtained with an exponential speedup as compared to a classic computer that would need to compute the result for each setting of the input bits separately. For a few applications, most notably for factoring integers [333], algorithms that can make use of the global property of the result have been developed. Another application for a quantum computer would be the simulation of quantum systems, an intractable problem on a classical computer [334].

One possibility for implementing qubits is to use atoms with unpaired nucleons $\left({ }^{1} \mathrm{H},{ }^{13} \mathrm{C},{ }^{15} \mathrm{~N}\right.$ $\left.{ }^{19} \mathrm{~F}{ }^{31} \mathrm{P},{ }^{35} \mathrm{Cl},{ }^{79} \mathrm{Cl}\right)$, i.e., atoms that have magnetic moments. If such atoms are located in a magnetic field the quantum state of the magnetic moments are split into different levels (Zeeman effect). Applying electromagnetic radiation at the resonance frequency of the nucleus gives rise to transitions of the magnetic moments between the differentiated levels. These transitions are accompanied by energy absorption or emission [335]. Accordingly the quantum state of the nucleus can be determined by probing with a pulse and measuring the absorption or emission response to the probing pulse. By applying carefully tailored sequences of radio frequency pulses the quantum state of a nucleus can be manipulated to implement the required unitary transformations for performing reversible logic operations [336].

For implementing a network of quantum logic gates it is necessary that qubits can interact. This is facilitated by direct or indirect coupling of the atoms that represent the qubits by connecting them in an appropriately chosen molecule. A NMR quantum computer is a single molecule; in practice, however, macroscopic ensembles of identical molecules are used. The qubits in different molecules are not coupled and because all molecules in the bulk material receive the same pulsed sequences they all perform identical operations. Conversely, the measurement of the output quantum state will provide an average signal from all molecules. While the output from 
the ensemble of molecules in the bulk material allows for a macroscopic response signal, this averaging is impeding the scale up of NMR quantum computing. The small energy differences that separate Zeeman levels prevent the possibility of preparing the ensemble of molecules in a pure ground state at feasible temperatures. In practice only a bias of the bulk material towards the ground state can be achieved, while the signals from the largely equally populated states cancel out. The weak bias serves as a pseudo-pure state and provides a detectable signal for determining the final state of a computation. The signal strength, however, falls off exponentially with the number of qubits employed. In principle this could be compensated by an exponential increase in number of molecules, but doing so would nullify the exponential advantage of quantum parallelism over classical computation [336]. Conceivably this difficulty could be mitigated to some extend with procedures for preparing the initial state of the ensemble with a stronger bias [337]. Algorithms that accept the exponential increase in resource requirements in order to simplify initialization have also been suggested [338], but there computational power is better compared to massive parallel computing methods such as DNA-computing (section 3.5) than to quantum computation.

So far room temperature liquid-state NMR quantum computing has been the only method to demonstrate nontrivial quantum computing, albeit with minimalist examples. Several quantum algorithms including parallel search [339, 340] and integer factorization have been realized. Shor's factorization algorithm [333], for example, was implemented by representing seven qubits with a ${ }^{13} \mathrm{C}$-labeled perfluorobutadienyl iron complex and the number 15 was factored into prime factors three and five [341].

Whether NMR quantum computing can be scaled up beyond minimalist examples is unclear. Some authors expect the limit practicable limit to lie below 20 qubit [336], while others estimate that 50 to 100 qubit could be realistic [337]. In between these predictions lies the threshold above which quantum computing could outperform classic computing [334]. The main difficulties in scaling up NMR quantum computing arise from a lack of error correction methods and the complications in preparing a suitable initial state [336]. Despite these hindrances to a generally scalable computing scheme it seems possible that NMR quantum computing could reach a level where it can be usefully applied. The path to this stage, however, may see molecules abandoned in favor of solid state materials [342, 343].

\section{INTERFACES, INTERCONNECTIONS, AND ARCHITECTURES}

Almost all molecular computing implementations that have been demonstrated up to now are on the level of individual operative devices. Interconnection technologies that enable the combination of molecular devices into circuits and networks are prerequisite to arriving at fully functional architectures. Even in conventional computing architectures communication among components is often a larger obstacle than computation. For many molecular technologies the interconnection of components into large architectures is an unsolved problem. Nature's molecular computers, e.g., single cell organisms, and neurons, however, point towards some possible solutions. The two key interconnection concepts found in natural molecular information processing are signaling molecules ('messengers') and direct conformational interaction. The former does not require spatial proximity between the communicating components, the latter can provide rapid signal transmission.

Related to the issue of interconnecting molecular components is the problem of interfacing a molecular architecture with the macroscopic level. Electrical and optical interfacing is available for many molecular systems, although optical interfacing in the visible light spectrum usually requires the introduction of special chromophore moieties. The recent availability of semiconductor devices (LEDs) with emission in the near ultra-violet [344] make optical methods increasingly more attractive for interfacing molecular materials with conventional electronic architectures. Many proposed molecular computing devices are not intended to function stand-alone, but as part of a hybrid molecular-conventional computing system, e.g., as a molecular co-processor [345] or a molecular memory $[172,346,173]$.

Some prototype architectures use the macroscopic amplification of signals also for interconnecting several molecular stages, because of a lack of molecular level interconnection. An early proposal along these lines was to the electronically controlled release of molecules into a buffer 
solution common to a number of molecular gates [347, 348] the individual gates would then select from the common buffer solution the signals relevant to them by molecular specificity.

Both light and electron transfer can serve for interconnecting molecular components as well as for micro-macro interfacing [349]. Aside from electrical and optical interfaces, magnetic and mechanical methods have also been investigated [350, 192]. Electrochemical interfacing of redox-reactions is another possibility. It has been applied, for example, to control a BelousovZhabotinsky medium with microelectrodes [351].

One of the difficulties underlying the afore mentioned interconnection problem arises from the fact, that the effort required for attaining precise prescriptive control increases rapidly with reduction of physical scale. Aside from homogeneous lattices, it is difficult to obtain extended architectures with nanoscale features. Top-down methods for assembling nanotechnology devices have poor throughput and seem ill suited for mass production [1]. Clearly an increase in component density with a concomitant increase in computational power density would be one of the main advantages of molecular information technologies.

Two main lines of research are under way for circumventing a production bottleneck that potentially could be more limiting to nanoscale technology than component size [1]. Firstly, computing schemes that do not require precise control over the underlying computing substrate are investigated, and, secondly, self-organization is studied as a bottom-up construction method.

\subsection{Diffusion and Transport}

Within biological cells many signals are transmitted by means of messenger molecules. The distribution of the messengers through diffusion is relatively fast within the volume of a cell, particularly if compared to the turnover rate of enzymes. The mean time for a molecule starting at a random location within a sphere of radius $r$ to reach a target at the center of the sphere increases with $r^{3}$, however [229]. For a volume larger than a few $\mu \mathrm{m}^{3}$, diffusion is therefore too slow to be a practical means of signal transmission. To cover larger distances, for example within an extended axon of a neuron, biological cells use intricate active transport mechanisms that use molecular recognition to shuttle signals along filaments. Protein targeting is a case in point [12]. At present it seems unlikely that this level of logistic organization could be obtained and maintained in a man-made device. Consequently, a molecular computing architecture relying solely on diffusion for signal transmission would need to be compartmentalized into $\mu \mathrm{l}$ volumes or confine the interacting species in a plane, such as a membrane, to speed up the mean time for a signal to arrive at its receptor.

\subsection{Uncontrolled Structures}

On the micro level the fine structure of many materials cannot be fabricated in accordance with a precise blue print. Nevertheless, several possibilities exist to exploit such structures for computational purposes:

- Components that perform within specification can be identified and only those are then selected for incorporation into a larger functional unit.

- Error correcting codes can introduce redundancy into stored data to compensate for faulty memory cells.

- Machine learning methods can be applied to learn how to make use of an existing set of components "as is".

- An attempt can be made to reorganize the existing structure to suit the application at hand, for example by burning out undesirable connections.

The first two methods above are part of the repertoire applied in conventional computing architectures and are commonly used in magnetic and optical disk drives.

It may be possible to configure circuits composed of several random molecular networks to perform simple logic operations.

Simulation results appear to indicate the possibility of configuring circuits composed of several random molecular networks to perform simple logic operations [352]. These simulation results are not specific to a particular material and therefore interesting, despite the fact that they were 
possibly motivated by experimental artefacts [132]. However, in order to arrive at functional circuits they assumed to know the detailed connections among the randomly connected molecules to program the random molecular networks with specific pulse sequences. Determining the interconnections appears difficult in practice and would not allow for changes occurring over the lifetime of the device. Furthermore, it is not clear how much hand-crafting was involved in arriving at structures that could yield functional circuits.

Bahar et al. proposed a framework based on Markov random fields and belief propagation that assumes a probabilistic computing substrate [353]. It thus allows to include variations in device fabrication, changes over device life-time, or disturbances from operating close to the thermal noise limit into consideration. Evolutionary computing provides another method for discovering useful functionality in random molecular structures [79].

The practicality of the procedures described in this section will depend on how the yield of functional components scales with the density and cost of the raw components. The more complex a local structure needs to be, the less likely it is that it will appear in an uncontrolled fabrication process. To achieve a high yield for complex structures fabrication needs to draw on the repeatability of self-organizing processes.

\subsection{Self-organization}

Macroscopic properties of molecular materials are often greatly influenced by the orientation of the molecules. Self-assembly can be a powerful technique to effect the desired arrangement of molecules at a speed suitable for mass production. The idea of using self-organization in the formation of information processing structures has been discussed at least since the cybernetics movement in the 1960s [354] and successful self-assembly of nanostructures had been demonstrated almost three decades before [91]. A growing understanding of the significance of non-covalent bonds for biological structures together with increasing success in the design of synthetic building blocks that self-assemble into supra molecular structures [84, 3] have highlighted the potential of using self-assembly for the fabrication of molecular devices.

Seeman demonstrated in extensive work the versatility of DNA for constructing nanostructures with a collection of topologically interesting two- and three-dimensional supramolecular assemblies [355]. These structures self-assemble from small carefully designed DNA duplexes with sticky ends (cf. figure 15B) and can be used as nanomechanical scaffolds [356]. In an approach reminiscent of Ulmer's suggestion to use two-dimensional protein tiles for self-assembly of random access memory (RAM) in a mosaic tile floor like fashion [27] the possibility of algorithmic control over the self-assembly process has been considered [36, 357]. Appropriate design of the building blocks should enables the self-organization of complex patterns. In theory even a memory array including its address decoder can self-assemble [358]. In practice however errors occurring during the assembly are likely to limit size and complexity of the patterns that can be realized [359]. It will be important to develop robust self-assembly algorithms that tolerate local faults without propagating them during the self-assembly. As long as effects from noise are confined and do not spread throughout the structure useful systems could be assembled even with a relatively high error rate. A memory with $10 \%$ faulty memory cells, for example, would still be useful. The established techniques for coping with disk drive errors, like a table keeping track of bad memory cells and error-correcting codes can be applied to provide a virtual fault free memory of reduced size. In general it appears likely that self-assembly will need to be combined with techniques such as those discussed in section 4.2 to achieve systems complex enough to possess of interesting functionality.

The flexibility of DNA self-assembly can be used to assemble other components by functionalizing them with single DNA strands [360]. Single stranded DNA can be covalently attached to the end of carbon nanotubes [116], for example, to facilitate the controlled self-assembly of nanotube structures that are more complex than the regular structures obtained through the application of an alternating current electric field [361]. Nanotubes themselves can also self-assemble from appropriately chosen DNA strands (yielding tubes of $\approx 25 \mathrm{~nm}$ diameter) and subsequently coated with silver to form conductive nano-wires [362]. The specific self-assembly of DNA molecules allows for specific interconnections between the DNA tubes and suitably prepared contact points [363]. Another thing to be noted is that random DNA networks have been obtained by selfassembly of polynucleotides [364]. For a recent review of the manifold applications of DNA for assembling nanostructures see [365]. 
Designing a set of self-assembly building blocks from DNA is particularly convenient, because the hybridization of DNA, i.e., the self-assembly of complementary single strands, is relatively predictable (cf. section 3.5) as compared to the self-assembly properties of proteins. Notwithstanding the fact that so far protein sequences cannot be designed for self-assembly, it is possible to build on proteins with known assembly properties [366] or antibodies can be developed to bind an intended target molecule [367]. Most natural self-assembly structures are composed of proteins. Among the advantages of self-assembled protein structures over DNA structures are increased stiffness. Potentially much more important, however, is another property of proteins. If the potential for conformational switching in proteins can be employed in the control of their self-assembly properties, as is commonly the case in natural protein structures, then protein is likely to allow for far more sophisticated self-assembly mechanisms than what can be expected from DNA building blocks [368].

A problem common to all self-assembly protocols is the fact that in each assembly step not all supramolecular structures may form correctly. After a sequence of assembly steps, numerous structures may have been formed that are defective, yet in their physiochemical properties so similar to the correctly formed structure, that it is difficult or outright impossible to discriminate between a faulty and a correct self-assembly product on a macroscopic level. If the self-assembled components are held together by relative weak forces, a self-healing process during which incorrectly assembled parts dissociate is sometimes possible. However, in structures with strong bonds a dissociation is too unlikely to allow for self-healing.

Coupling of form and function together with shape based interaction [212], introduces an automatic error control in biological systems. A protein that is missfolded or mutated and thus is likely not to function correctly, will have an altered shape and hence is not likely to be integrated into the protein interaction network.

To arrive at complex extended architectures an energy driven development and growth process is required. Development of a functional structure should restrict the search space to avoid a de nuovo design for every instance, but at the same time provide the flexibility to accommodate a wide variation in boundary conditions and to adapt the developing structure to the specific demands encountered during growth [369, 370, 371]. Growth processes in nature are highly adaptive and, as an essential property for evolution, are flexible with respect to variations in the parameters of the growing system as well as adaptive to different environmental conditions. Furthermore, the integration density in three dimensions [372] far exceeds anything one could reasonably hope to achieve with top-down assembly.

\section{PERSPECTIVES}

At present there is good progress in bulk molecular materials with devices reaching the market place. A first system using a protein (BR) as main active component is commercially available since several years $[157,155]$ and numerous patents for the use of this protein in information technology applications have been filed [156]. Single molecule (true molecular) devices however are still at an early laboratory stage. Tremendous challenges lie in the path to a widely applicable molecular information technology based on single molecule devices. Yet these challenges are matched by the opportunities they open up. Nature relies heavily on molecular structures for information processing. It provides a proof of concept and is an invaluable source for hints on how to overcome the obstacles.

A key area that needs to be addressed is the development of assembly routes capable of delivering extended heterogenous nanostructures at a time scale that does not increase proportional with the component count. This will require a novel engineering approach that does not depend on the direct control of individual components. The spontaneous generation of supramolecular architectures from molecular components may provide a path to self-organized functional architectures [80]. Self-organizing processes ranging from self-assembly to growth and development will play a central role in mimicking the high integration density of nature's information processing architectures in artificial devices. In the long run a dynamic exchange of components in the functional system is desirable to insure long term reliability. Once a technology for growing nanostructures is in place, self-repair will come into sight as an extension of the growth process. The ultimate form of self-repair would be self-reproduction. Conceivably technical structures could be implemented as virus like systems that rely on a host cell system for reproduction, thus largely avoiding the overhead associated with a autonomously self-reproducing system. 
The second, and no less important issue, is the development of computing concepts that exploit rather than suppress the physics of the materials used for their implementation. Combinatorial synthesis is now applied in the search for new materials such as, luminescent materials, polymer semiconductors, high temperature superconductors, and novel magnetic materials. At the same time techniques for producing biomaterials and manufacturing nano-materials are rapidly developing. The potential for discovering novel compounds with properties of commercial interest is high. For example, about 18 million organic compounds are known today - a negligible number if compared to the space of possible organic molecules, estimated to $10^{63}$ substances [296]. Supramolecular chemistry, by means of self-assembly of components further increases the space of possibilities [373].

In the near future we will see materials with unprecedented characteristics arriving at an increasing rate. But so far we are not on a path to harnessing these new materials in an effective way for increased computational power. Computer science has to change its requirements. Trying to coerce any computational substrate into acting as fast boolean switching network is likely to largely relinquish the true capabilities arising from broadening the material basis of computation to include molecular and nano materials. Novel computing concepts need to match the new materials [73]. Cellular automata and neural networks provide possible starting points for parallel computing schemes more suitable to molecular implementation. The development of simple artificial biochemical 'neurons' [374, 375] may provide an approach to computational infrastructure more closely aligned with the natural capabilities of molecular materials than the quest for logic gates.

What are the opportunities opened up by molecular materials? Many information processing problems effectively addressed by cells and organisms are out of reach for present-day computers. Nevertheless, early application domains for molecular technology are not likely to compete with conventional technology in the near future as regard to raw computing power. Early applications are likely to fall into two categories using biocompatibility and high integration density, respectively. Bioimmersive computing requires artificial information processing device at the cellular level. Fusion of signals from multiple receptors incorporated into living cells and dynamic control of a cell's function, behavior, or expression products for medical ('living pills') and environmental monitoring ('living sensors') are potential applications. Engineered microbes [376, 377] with bioimmersive control structures may be employed for detoxification or for the accumulation of rare substances.

The second domain is coherent perception-action under real-time constraints in microrobots. Molecular technology may in the future be able to deliver the high integration density and low power consumption that is required to narrow the gap between simple biological organisms and present artificial devices in size, performance, and adaptability [378, 379, 380].

Finally, it is worth pointing out that integrating new devices into existing technology can be expected to be slow. Despite the close analogy between transistors and vacuum tubes [381] and the undisputed advantages of the transistors in size and power consumption, it took ten years from the announcement of the transistor [382] to its appearance in a practical computer [383]. It will likely take much longer until novel computing schemes and architectures will become standard components in computers.

Until now the history of science has seen a sharpening of the line separating inanimate matter from animate matter [384]. I am disposed to think that we are at the final stage of this process and that in the coming decades this distinction will become increasingly blurred.

\section{ACKNOWLEDGMENTS}

The invitation to write this article was originally extended to Michael Conrad. He had prepared a detailed outline, but his struggle with cancer prevented him from further pursuing this project. Nevertheless, his influence is manifest in many parts of this article and is gratefully acknowledged. Discussions with Andrew Adamatzky, Srinandan Dasmahapatra, Peter Dittrich and Jürgen Ficker were helpful. This article is based in part upon work supported by NASA under Grant No. NCC2-1189. 


\section{References}

[1] R. Compañó. Trends in nanoelectronics. Nanotechnology, 12:85-88, 2001.

[2] The international technology roadmap for semiconductors, 2003. Available at http://www.itrs.net.

[3] G. M. Whiteside, J. P. Mathias, and C. T. Seto. Molecular self-assembly and nanochemistry: A chemical strategy for the synthesis of nanostructures. Science, 254:1312-1319, 1991.

[4] H. H. P. Yiu, C. H. Botting, N. P. Botting, and P. A. Wright. Size selective protein adsorption on thiolfunctionalised SBA-15 mesoporous molecular sieve. Phys. Chem. Chem. Phys., 3:2983-2985, 2001.

[5] C. Koch. Computation and the single neuron. Nature, 385:pp. 207-210, 1997.

[6] J. Stock and M. Levit. Signal transdution: Hair brains in bacterial chemotaxis. Current Biology, 10:R11R14, 2000.

[7] T. S. Gardner, C. R. Cantor, and J. J. Collins. Construction of a genetic toggle switch in Escherichia coli. Nature, 403:339-342, 2000.

[8] M. R. Atkinson, M. A. Savageau, J. T. Myers, and A. J. Ninfa. Development of genetic circuitry exhibiting toggle switch or oscillatory behavior in Escherichia coli. Cell, 113:597-607, 2003.

[9] L. M. Adleman. Molecular computation of solutions to combinatorial problems. Science, 266:1021-1024, 1994.

[10] M. Amos, G. Paun, G. Rozenberg, and A. Salomaa. Topics in the theory of DNA computing. Theoretical Computer Science, 287:3-38, 2002.

[11] R. F. Gesteland and J. F. Atkins. Recoding: Dynamic reprogramming of translation. Annu. Rev. Biochem., 65:741-768, 1996.

[12] L. Stryer. Biochemistry. W. H. Freeman, New York, 1988.

[13] P. M. Ajayan. Nanotubes from carbon. Chem. Rev., 99:1787-1799, 1999.

[14] M. Terrones. Science and technology of the twenty-first century: Synthesis, properties, and applications of carbon nanotubes. Annu. Rev. Mater.Res., 33:419-501, 2003.

[15] N. Hampp, C. Bräuchle, and D. Oesterhelt. Mutated bacteriorhodopsins: Competitive materials for optical information processing? Materials Research Society (MRS) Bulletin, 17:56-60, 1992.

[16] N. Ban, P. Nissen, J. Hansen, P. B. Moore, and T. A. Steitz. The complete atomic structure of the large ribosomal subunit at 2.4 å resolution. Science, 289:905-920, 2000.

[17] W. R. Wikoff and J. E. Johnson. Virus assembly: Imaging a molecular machine. Current Biology, 9:R296R300, 1999.

[18] M. Conrad. Amplification of superpositional effects through electronic-conformational interactions. Chaos, Solitons \&f Fractals, 4:423-438, 1994.

[19] E. A. Liberman. Cell as a molecular computer (MCC). Biofizika, 17:932-943, 1972.

[20] M. Conrad. Information processing in molecular systems. Currents in Modern Biology (now BioSystems), $5: 1-14,1972$.

[21] M. N. Vaintsvaig and Ye. A. Liberman. Molecular computer-II. formal description (system of operation). Biofizika, 18(5):939-941, 1973. Pages 1003-1006 in the English translation of Biofizika.

[22] E. A. Liberman. Analog-digital molecular cell computer. BioSystems, 11:111-124, 1979.

[23] F. F. Seelig and O. E. Rössler. A chemical reaction flip-flop with one unique switching input. Zeitschrift für Naturforschung, 27b:1441-1444, 1972.

[24] O. E. Rössler. Chemical automata in homogeneous and reaction-diffusion kinetics. In M. Conrad, W. Güttinger, and M. Dal Cin, editors, Physics and Mathematics of the Nervous System, volume 4 of Lecture Notes in Biomathematics, pages 399-418. Springer, Heidelberg, 1974.

[25] A. Aviram and M. A. Ratner. Molecular rectifiers. Chem. Phys. Lett., 29:277-283, 1974.

[26] S. Yanchinski. And now-the biochip. New Scientist, pages 68-71, 14 January 1982.

[27] Anonymous. Molecular electronics: Beyond the silicon chip. Emerging technologies no. 9, Technical Insights, Fort Lee, NJ, September 1983.

[28] R.C. Haddon and A. A. Lamola. The molecular electronic device and the biochip computer: present status. Proc. Natl. Acad. Sci. USA, 82(7):1874-1878, 1985.

[29] M. Conrad. On design principles for a molecular computer. Commun. ACM, 28:464-480, 1985.

[30] M. Conrad. Quantum mechanics and molecular computing: Mutual implications. Int. J. Quantum Chem: Quantum Biol. Symp., 15:287-301, 1988.

[31] M. Conrad. Molecular computing. In M. C. Yovits, editor, Advances in Computers, volume 31, pages 235-324. Academic Press, Boston, 1990.

[32] D. E. Atkinson. Conformational change and modulation of enzyme activity. In G. C. Quarton, T. Melnechuk, and F. O. Schmitt, editors, The Neurosciences, pages 123-130. Rockefeller University Press, New York, 1967.

[33] M. Conrad. Microscopic-macroscopic interface in biological information processing. BioSystems, 16:345-363, 1984.

[34] M. Conrad. Quantum molecular computing: the self-assembly model. Int. J. Quant. Chem.: Quant. Biol. Symposium, 19:125-143, 1992.

[35] M. Conrad. Emergent computation through self-assembly. Nanobiology, 2:5-30, 1993.

[36] E. Winfree, F. Liu, L. A. Wenzler, and N. C. Seeman. Design and self-assembly of two-dimensional DNA crystals. Nature, 394:137-138, 1998.

[37] R. Landauer. Fundamental limitations in the computational process. Berichte der Bunsen-Gesellschaft, 80(11):1048-1059, 1976. 
[38] M. Conrad and K.-P. Zauner. Conformation-based computing: a rational and a recipe. In T. Sienko, A. Adamatzky, N. Rambidi, and M. Conrad, editors, Molecular Computing, chapter 1, pages 1-31. MIT Press, Cambridge, MA, 2003.

[39] G. B. Street and T. C. Clarke. Conducting polymers: A review of recent work. IBM J. Res. E Dev., 25(1):51-57, 1981

[40] A. Diaz. Electrochemical preparation and characterization of conducting polymers. Chemica Scripta, 17:145-148, 1981.

[41] Anonymous. Conducting polymer R\&D continues to grow. Chemical \& Engineering News, pages 29-33, April 191982.

[42] F. L. Carter. Problems and prospects of future electroactive polymers and "molecular" electronic devices. Technical Report NRL Memo Report 3960, US Naval Research Laboratory, Washington DC, 1979.

[43] C. J. Drury, C. M. J. Mutsaers, C. M. Hart, M. Matters, and D. M. de Leeuw. Low-cost all-polymer integrated circuits. Appl. Phys. Lett., 73(1):108-110, 1998.

[44] G. H. Gelinck, T. C. T. Geuns, and D. M. de Leeuw. High-performance all-polymer integrated circuits. Appl. Phys. Lett., 77(10):1487-1489, 2000.

[45] W. Fix, A. Ullmann, J. Ficker, and W. Clemens. Fast polymer integrated circuits. Appl. Phys. Lett., 81(9):1735-1737, 2002.

[46] J. N. Harris, P. E. Gray, and C. L. Searle. Digital Transitor Circuits, chapter 5, pages 131-160. John Wiley \& Sons, New York, 1966.

[47] R. P. Feynman. Feynman Lectures on Computation. Perseus Publishing, Cambridge, MA, 1996. T. Hey and R. W. Allen, Eds.

[48] H. Taub and D. Schilling. Digital Integrated Electronics, chapter 9, pages 278-321. McGraw-Hill, Singapore, 1977.

[49] M. A. Harrison. Introduction to switching and automata theory, chapter 9,10, pages 272-305. McGraw-Hill, New York, 1965.

[50] M. L. Minsky. Computation: Finite and Infinite Machines. Prentice-Hall, Englewood Cliffs, N.J., 1967.

[51] M. A. Arbib. Brains, Machines and Mathematics. McGraw-Hill, New York, 1964.

[52] R. W. Keyes. Electronics in large systems. In K. Sienicki, editor, Molecular Electronics and Molecular Electronic Devices, volume I, chapter 1, pages 1-30. CRC Press, Boca Raton, 1993.

[53] R. D. Isaac. The future of CMOS technology. IBM J. Res. ES Dev., 44(3):369-378, 2000.

[54] P. M. Solomon. Device innovation and material challenges at the limits of CMOS technology. Annu. Rev. Mater. Sci., 30:681-697, 2000.

[55] J. W. McPherson. Scaling-induced reductions in CMOS reliability margins and the escalating need for increased design-in reliability efforts. In Proceedings of the International Symposium on Quality Electronic Design (ISQED'01). IEEE Computer Society, Los Alamitos, 2001.

[56] A. M. Turing. On computable numbers with an application to the Entscheidungsproblem. In Proceedings of the London Mathematical Society, volume 42, pages 230-265, 1937. Corrections, Ibid vol. 43 (1937), pp. 544-546. Reprinted in The Undecideable, M. Davis, ed., Raven Press, New York, 1965.

[57] M. Davis. Computability \& Unsolvablity, chapter 1, pages 3-24. McGraw-Hill, New York, 1958.

[58] G. Kampis. Self-Modifying Systems in Biology and Cognitive Science, page 473. Pergamon Press, Oxford, 1991.

[59] H. J. Bremermann. Complexity of automata, brains and behavior. In M. Conrad, W. Güttinger, and M. Dal Cin, editors, Physics and Mathematics of the Nervous System, pages 304-331. Springer, Heidelberg, 1974.

[60] H. J. Bremermann. Complexity and transcomputability. In The Encyclopaedia of Ignorance, pages 167-174. Pocket Books, New York, 1978.

[61] G. J. Klir. Facets of Systems Science, volume 7 of International Series on Systems Science and Engineering, pages 113-134. Plenum Press, New York, 1991.

[62] V. Pratt. Thinking Machines. Basil Blackwell, Oxford, 1987.

[63] D. A. Grier. Human computers: the first pioneers of the information age. Endeavour, 25(1):28-32, 2001.

[64] K.-P. Zauner and M. Conrad. Molecular approach to informal computing. Soft Computing, 5(1):39-44, 2001.

[65] G. R. Stibitz and J. A. Larrivee. Mathematics and Computers, page 151. McGraw-Hill, New York, 1957.

[66] M. Conrad. Scaling of efficiency in programmable and non-programmable systems. BioSystems, 35:161-166, 1995.

[67] A. W. Burks. Von Neumann's self-reproducing automata. In A. W. Burks, editor, Essays on Cellular Automata, chapter 10, pages 3-64. University of Illinois Press, Urbana, 1970.

[68] C. S. Lent, B. Isaksen, and M. Lieberman. Molecular quantum-dot cellular automata. J. Am. Chem. Soc., 125:1056-1063, 2003.

[69] B. J. MacLennan. Field computation in natural and artificial intelligence. Information Sciences, 119:73-89, 1999.

[70] H. Abelson, D. Allen, D. Coore, C. Hanson, E. Rauch, G. J. Sussman, and R. Weiss. Amorphous computing. Communication of the ACM, 43(5):74-82, 2000.

[71] M. Conrad. The price of programmability. In R. Herken, editor, The Universal Turing Machine: A Fifty Year Survey, pages 285-307. Oxford University Press, New York, 1988.

[72] D. Partridge. Non-programmed computation. Communications of the ACM, 43:293-302, 2000. 
[73] K.-P. Zauner. From prescriptive programming of solid-state devices to orchestrated self-organisation of informed matter. In J.-P. Banâtre, J.-L. Giavitto, P. Fradet, and O. Michel, editors, Pre-Proceedings of UPP'04, Unconventional Programming Paradigms, 15-17 September, pages 123-128, Le Mont Saint-Michel, France, 2004. ERCIM. Available at: http://eprints.ecs.soton.ac.uk/10407/.

[74] R. Landauer. Irreversibility and heat generation in the computing process. IBM Journal, 5(3):183-191, 1961.

[75] W. B. Langdon. How many good programs are there? How long are they? In J. Rowe et al., editors, Foundations of Genetic Algorithms FOGA-7, Torremolinos, 4-6 September, 2002. Morgan Kaufmann.

[76] W. B. Langdon. The distribution of reversible functions is normal. In R. Riolo, editor, Genetic Programming Theory and Practice, Ann Arbor, 15-17 May, Proceedings, Dordrecht, 2003. Kluwer Academic Publishers.

[77] K.-P. Zauner and M. Conrad. Parallel computing with DNA: toward the anti-universal machine. In H.M. Voigt, W. Ebeling, I. Rechenberg, and H.-P. Schwefel, editors, Parallel Problem Solving from Nature: PPSN IV, volume 1141 of Lecture Notes in Computer Science, pages 696-705, Berlin, September 1996. Springer-Verlag, Berlin.

[78] J. O. Pfaffmann and K.-P. Zauner. Scouting context-sensitive components. In D. Keymeulen, A. Stoica, J. Lohn, and R. S. Zebulum, editors, The Third NASA/DoD Workshop on Evolvable Hardware-EH-2001, pages 14-20, Long Beach, 12-14 July 2001, 2001. IEEE Computer Society, Los Alamitos.

[79] J. F. Miller and K. Downing. Evolution in materio: Looking beyond the silicon box. In 2002 NASA/DoD Conference on Evolvable Hardware (EH'O2), pages 167-176, July 15 - 18, 2002, Alexandria, Virginia, 2002. IEEE.

[80] J.-M. Lehn. Supramolecular chemistry: from molecular information towards self-organization and complex matter. Reports on Progress in Physics, 67:249-265, 2004.

[81] M. O. Magnasco. Chemical kinetics is turing universal. Phys. Rev. Lett., 78:1190-1193, 1997.

[82] D. T. Gillespie. Approximate accelerated stochastic simulation of chemically reacting systems. Journal of Chemical Physics, 115(4):1716-1733, 2001.

[83] P. Dittrich, J. Ziegler, and W. Banzhaf. Artificial chemistries-a review. Artificial Life, 7(3):225-275, 2001.

[84] J. M. Lehn. Supramolecular chemistry—scope and perspectives: Molecules, supermolecules and molecular devices. Angewandte Chemie, Int. Ed. Engl., 27:90-112, 1988.

[85] W. Shockley. A unipolar field-effect transistor. Proc. I.R.E., 40:1365-1376, 1952.

[86] F. Ebisawa, T. Kurokawa, and S. Nara. Electrical properties of polyacetylene/polysiloxane interface. $J$. Appl. Phys., 54(6):3255-3259, 1983.

[87] J. H. Burroughes, R. H. Friend, and P. C. Allen. Field-enhanced conductivity in polyacetylene - construction of a field-effect transistor. J. Phys. D: Appl. Phys., 22(7):956-958, 1989.

[88] C. D. Dimitrakopoulos and D. J. Mascaro. Organic thin-film transistors: A review of recent advances. IBM J. Res. \& Dev., 45(1):11-27, 2001.

[89] Y. Wada. Prospects for single molecule information processing devices. Current Applied Physics, 1:28-38, 2001.

[90] E. E. Polymeropoulos, D. Möbius, and H. Kuhn. Monolayer assemblies with functional units of sensitizing and conducting molecular-components-photo-voltage, dark conduction and photoconduction in systems with aluminum and barium electrodes. Thin Solid Films, 68(1):173-190, 1980.

[91] K. B. Blodgett. Films built by depositing successive monomolecular layers on a solid surface. J. Am. Chem. Soc., 57:1007-1022, 1935.

[92] M. C. Petty. Langmuir-Blodgett Films: An Introduction. Cambridge University Press, Cambridge, 1996.

[93] M. Fujihira, K. Nishiyama, and H. Yamada. Photoelectrochemical responses of optically transparent electrodes modified with Langmuir-Blodgett-films consisting of surfactant derivatives of electron-donor, acceptor and sensitizer molecules. Thin Solid Films, 132(1-4):77-82, 1985.

[94] R. M. Metzger, B. Chen, U. Höpfner, , M. V. Lakshmikantham, D. Vuillaume, T. Kawai, X. Wu, H. Tachibana, T. V. Hughes, H. Sakurai, J. W. Baldwin, C. Hosch, M. P. Cava, L. Brehmer, and G. J. Ashwell. Unimolecular electrical rectification in hexadecylquinolinium tricyanoquinodimethanide. J. Am. Chem. Soc., 119(43):10455-10466, 1997.

[95] R. M. Metzger. All about (N-hexadecylquinolin-4-ium-1-yl)methylidenetricyanoquinodimethanide, a unimolecular rectifier of electrical current. J. Mater. Chem., 10(1):55-62, 2000.

[96] H. V. Malmstadt and C. G. Enke. Digital Electronics for Scientists. W. A. Benjamin, New York, 1969.

[97] S. Ami and C. Joachim. Logic gates and memory cells based on single $\mathrm{C}_{60}$ electromechanical transistors. Nanotechnology, 12:44-52, 2001.

[98] R. C. Haddon, A. S. Perel, R. C. Morris, T. T. M. Palstra, A. F. Hebard, and R. M. Fleming. C-60 thin-film transistors. Appl. Phys. Lett., 67(1):121-123, 1995.

[99] F. Garnier, R. Hajlaoui, and A. Yassar. All-polymer field-effect transistor realized by printing techniques. Science, 265(5179):1684-1686, 1994.

[100] J. Tate, J.A. Rogers, C.D.W. Jones, B. Vyas, D.W. Murphy, W. J. Li, Z. A. Bao, R. E. Slusher, A. Dodabalapur, and H. E. Katz. Anodization and microcontact printing on electroless silver: Solution-based fabrication procedures for low-voltage electronic systems with organic active components. Langmuir, 14:6054-6060, 2000.

[101] J. A. Rogers, Z. Bao, K. Baldwin, A. Dodabalapur, B. Crone, V. R. Raju, V. Kuck, H. Katz, K. Amundson, J. Ewing, and P. Drzaic. Paper-like electronic displays: Large-area rubber-stamped plastic sheets of electronics and microencapsulated electrophoretic inks. Proc. Nat. Acad. US, 98(9):4835-4840, 2001.

[102] J. A. Rogers. Rubber stamping for plastic electronics and fiber optics. MRSBulletin, 26(7):530-534, 2001. 
[103] Z. Bao, J. A. Rogers, and H. E. Katz. Printable organic and polymeric semiconducting materials and devices. J. Mater. Chem., 9:1895-1904, 1999.

[104] B. K. Crone, A. Dodabalapur, R. Sarpeshkar, R. W. Filas, Y. Y. Lin, Z. Bao, J. H. O’Neill, W. Li, and H. E. Katz. Design and fabrication of organic complementary circuits. J. Appl. Phys., 89(9):5125-5132, 2001.

[105] R. Want. RFID - a key to automating everything. Scientific American, 290(1):56-65, 2004.

[106] P. F. Baude, D. A. Ender, M. A. Haase, T. W. Kelley, D. V. Muyres, and S. D. Theiss. Pentacene-based radio-frequency identification circuitry. Applied Physics Letters, 82(22):3964-3966, 2003.

[107] P. F. Baude, D. A. Ender, T. W. Kelley, M. A. Haase D. V. Muyres, and S. D. Theiss. Organic semiconductor RFID transponders. In Electron Devices Meeting, December 8-10, 2003. IEDM '03 Technical Digest, pages 191-194. IEEE International, 2003.

[108] G. H. Gelinck, H. E. A. Huitema, E. Van Veenendaal, E. Cantatore, L. Schrijnemakers, J. B. P. H. Van der Putten, T. C. T. Geuns, M. Beenhakkers, J. B. Giesbers, B.-H. Huisman, E. J. Meijer, E. M. Benito, F. J. Touwslager, A. W. Marsman, B. J. E. Van Rens, and D. M. De Leeuw. Flexible active-matrix displays and shift registers based on solution-processed organic transistors. Nature Materials, 3:106-110, 2004.

[109] P. C. H. Li and D. J. Harrison. Transport, manipulation, and reaction of biological cells on-chip using electrokinetic effects. Analytical Chemistry, 69(8):1564-1568, 1997.

[110] J. M. Cooper. Towards electronic petri dishes and picolitre-scale single-cell technologies. Trends in Biotechnology, 17:226-230, 1999.

[111] R. Saito, G. Dresselhaus, and M.S. Dresselhaus. Physical properties of carbon nanotubes. Imperial College Press, London, 1998.

[112] S. Iijima. Helical microtubules of graphitic carbon. Nature, 354:56-58, 1991.

[113] N. Hamada, S. Sawada, and A. Oshiyama. New one-dimensional conductors: Graphitic microtubules. Phys. Rev. Lett., 68(10):1579-1581, 1992.

[114] S. J. Tans, A. R. Verschueren, and C. Dekker. Room-temperature transistor based on a single carbon nanotube. Nature, 393(6680):49-52, 1998.

[115] S. E. Baker, W. Cai, T. L. Lasseter, K. P. Weidkamp, and R. J. Hamers. Covalently bonded adducts of deoxyribonucleic acid (DNA) oligonucleotides with single-wall carbon nanotubes: Synthesis and hybridization. Nano Letters, 2(12):1413-1417, 2002.

[116] C. Dwyer, M. Guthold, M. Falvo, S. Washburn, R. Superfine, and D. Erie. DNA-functionalized single-walled carbon nanotubes. Nanotechnology, 13:601-604, 2002

[117] A. Star, J. F. Stoddart, D. Steuerman, M. Diehl, A. Boukai, E. W. Wong, X. Yang, S.-W. Chung, H. Choi, and J. R. Heath. Preparation and properties of polymer-wrapped single-walled carbon nanotubes. Angew. Chem. Int. Ed., 40(9):1721-1725, 2001.

[118] R. J. Chen, Y. Zhang, D. Wang, and H. Dai. Noncovalent sidewall functionalization of single-walled carbon nanotubes for protein immobilization. J. Am. Chem. Soc., 123:3838-3839, 2001.

[119] K. Keren, R. S. Berman, E. Buchstab, U. Sivan, and E. Braun. DNA-templated carbon nanotube field-effect transistor. Science, 302:1380-1382, 2003.

[120] A. Bachtold, P. Hadley, T. Nakanishi, and C. Dekker. Logic circuits with carbon nanotube transistors. Science, 294:1317-1320, 2001.

[121] M. S. Dresselhaus, G. Dresselhaus, and P. C. Eklund. Science of Fullerenes and Carbon Nanotubes : Their Properties and Applications. Academic Press, San Diego, 1996.

[122] M. S. Dresselhaus, G. Dresselhaus, and P. Avouris, editors. Carbon Nanotubes: Synthesis, Structure, Properties, and Applications, volume 80 of Topics in Applied Physics. Springer, New York, 2001.

[123] C. P. Collier, E. W. Wong, M. Belohradský, F. M. Raymo, J. F. Stoddart, P. J. Kuekes, R. S. Williams, and J. R. Heath. Electronically configurable molecular-based logic gates. Science, 285:391-394, 1999.

[124] H. V. Malmstadt and C. G. Enke. Digital Electronics for Scientists, chapter 4.5, pages 151-160. W. A. Benjamin, New York, 1969.

[125] J. Millman and H. Taub. Pulse and Digital Circuits, chapter 13, pages 392-428. McGraw-Hill, New York, 1956.

[126] A. R. Pease, J. O. Jeppesen, J. F. Stoddart, Y. Luo, C. P. Collier, and J. R. Heath. Switching devices based on interlocked molecules. Acc. Chem. Res., 34:433-444, 2001.

[127] J. M. Tour, M. Kosaki, and J. M. Seminario. Molecular scale electronics: a synthetic/computational approach to digital computing. J. Amer. Chem. Soc., 120:8486-8493, 1998.

[128] J. Chen, M. A. Reed, A. M. Rawlett, and J. M. Tour. Large on-off ratio and negative differential resistance in a molecular electronic device. Science, 286:1550-1552, 1999.

[129] J. M. Seminario, L. E. Córdova, and P. A. Derosa. An ab initio approach to the calculation of currentvoltage characteristics of programmable molecular devices. Proceedings of the IEEE, 91(11):1958-1975, 2003.

[130] Z. J. Donhauser, B. A. Mantooth, K. F. Kelly, L. A. Bumm, J. D. Monnell, J. J. Stapleton, D. W. Price Jr., A. M. Rawlett, D. L. Allara, J. M. Tour, and P. S. Weiss. Conductance switching in single molecules through conformational changes. Science, 292:2303-2307, 2001.

[131] A. M. Rawlett, T. J. Hopson, L. A. Nagahara, R. K. Tsui, G. K. Ramachandran, and S. M. Lindsay. Electrical measurements of a dithiolated electronic molecule via conducting atomic force microscopy. Applied Physics Letters, 81(16):3043-3045, 2002.

[132] J. M. Tour, L. Cheng, D. P. Nackashi, Y. Yao, A. K. Flatt, S. K. St. Angelo, T. E. Mallouk, and P. D. Franzon. Nanocell electronic memories. J. Am. Chem. Soc., 125:13279-13283, 2003.

[133] K. Szacilowski and Z. Stasicka. Molecular switches based on cyanoferrate complexes. Coordination Chemistry Reviews, 229:17-26, 2002. 
[134] F. Remacle, R. Weinkauf, D. Steinitz, K. L. Kompa, and R. D. Levine. Molecular logic by optical spectroscopy with output transfer by charge migration along a peptide. Chemical Physics, 281:363-372, 2002.

[135] H. Bouas-Laurent and H. Dürr. Organic photochromism. Pure Appl. Chem., 73(4):639-665, 2001.

[136] Y. Hirshberg. Reversible formation and eradication of colors by irradiation at low temperatures. a photochemical memory model. J. Am. Chem. Soc., 78:2304-2312, 1956.

[137] A. P. de Silva, D. B. Fox, T. S. Moody, and S. M. Weir. The development of molecular fluorescent switches. Trends in Biotechnology, 19(1):29-34, 2001.

[138] A. P. de Silva, N. H. Q. Gunaratne, and C. P. McCoy. A molecular photoionic AND gate based on fluorescent signalling. Nature, 364:42-44, 1993.

[139] A. P. de Silva and N. D. McClenaghan. Molecular-scale logic gates. Chem. Eur. J. 2004, 10, 574 586, 10:574-586, 2004.

[140] G. Dilek and E. U. Akkaya. Novel squaraine signalling Zn(II) ions: three-state fluorescence response to a single input. Tetrahedron Letters, 41:3721-3724, 2000.

[141] S. Tyagi and F. R. Kramer. Molecular beacons: probes that fluoresce upon hybridization. Nature Biotech., 14(3):303-308, 1996.

[142] F. C. Simmel and B. Yurke. A DNA-based molecular device switchable between three distinct mechanical states. Applied Physics Letters, 80(5):883-885, 2002.

[143] F. C. Simmel and B. Yurke. Using DNA to construct and power a nanoactuator. Physical Review E, 63:041913-1-5, 2001.

[144] A. Saghatelian, N. H. Völcker, K. M. Guckian, V. S.-Y. Lin, and M. R. Ghadiri. DNA-based photonic logic gates: AND, NAND, and INHIBIT. J. Am. Chem. Soc., 125:346-347, 2003.

[145] R. P. Fahlman, M. Hsing, C. S. Sporer-Tuhten, and D. Sen. Duplex pinching: A structural switch suitable for contractile dna nanoconstructions. Nano Letters, 3(8):1073-1078, 2003.

[146] M. S. P. Sansom and H. Weinstein. Hinges, swivels and switches: the role of prolines in signalling via transmembrane $\alpha$-helices. TiBS, 21:445-451, November 2000.

[147] Y. Zhao, V. Yarov-Yarovoy, T. Scheuer, and W. A. Catterall. A gating hinge in $\mathrm{Na}^{+}$channels: A molecular switch for electrical signaling. Neuron, 41:859-865, 2004.

[148] P. N. Hengen, I. G. Lyakhov, L. E. Stewart, and T. D. Schneider. Molecular flip-flops formed by overlapping Fis sites. Nucleic Acids Research, 31(22):6663-6673, 2003.

[149] Annonymous. Soviet general purpose optical computer technology: Progress and prospects. Technical report, USA Directorate of Intelligence (CIA), 1989. Released in sanitized form, March 2000: http://www.foia.ucia.gov/princeton_collection.htm.

[150] V. Y. Bazhenov, M. S. Soskin, V. B. Taranenko, and M. V. Vasnetsov. Biopolymers for real-time optical processing. In H. H. Arsenault, T. Szoplik, and B. Macukow, editors, Optical processing and computing, chapter 4, pages 103-144. Academic Press, San Diego, 1989.

[151] M. E. Conrad, R. R. Birge, G. J. Iafrate, R. S. Potember, F. R. Salemme, and H. T. Tien. Soviet and East European research related to molecular electronics. Technical report, Science Applications International Corporation, McLean, VA, 1990.

[152] K. Ludmann, C. Gergely, and G. Varo. Kinetic and thermodynamic study of the bacteriorhodopsin photocycle over a wide pH range. Biophysical J., 75(6):3110-3119, 1998.

[153] V. Sundström. Light in elementary biological reactions. Progress in Quantum Electronics, 24:187-238, 2000.

[154] R. R. Birge. Photophysics and molecular electronic applications of the rhodopsins. Ann. Rev. Phys. Chem., 41:683-733, 1990 .

[155] N. Hampp. Bacteriorhodopsin as a photochromic retinal protein for optical memories. Chem. Rev., 100:1755-1776, 2000.

[156] N. A. Hampp. Bacteriorrhodopsin: mutating a biomaterial into an optoelectronic material. Appl. Microbiol. Biotechnol., 53:633-639, 2000.

[157] T. Juchem and N. Hampp. Interferometric system for non-destructive testing based on large diameter bacteriorhodopsin films. Optics and Lasers in Engineering, 34:87-100, 2000.

[158] F. T. Hong. Interfacial photochemistry of retinal proteins. Progress in Surface Science, 62:1-237, 1999.

[159] F. T. Hong. Do biomolecules process information differently than synthetic organic-molecules. BioSystems, 27(4):189-194, 1992.

[160] U. Haupts, J. Tittor, and D. Oesterhelt. Closing in on bacteriorhodopsin: Progress in understanding the molecule. Annu. Rev. Biophys. Biomol. Struct., 28:367-399, 1999.

[161] N. Hampp, R. Thoma, D. Oesterhelt, and C. Bräuchle. Biological photochrome bacteriorhodopsin and its genetic variant asp96 $\rightarrow$ asn as media for optical pattern recognition. Applied Optics, 31(11):1834-1841, 1992.

[162] B. Ni, M. Chang, A. Duschl, J. Lanyi, and R. Needleman. An efficient system for synthesis of bacteriorhodopsin in halobacterium halobium. Gene, 90:169-172, 1990.

[163] F. T. Hong. Fundamentals of photoelectric effects in molecular electronic thin film devices: applications to bacteriorhodopsin-based devices. BioSystems, 35:117-121, 1995.

[164] T. Miyasaka, K. Koyama, and I. Itoh. Quantum conversion and image detection by a bacteriorhodopsinbased artificial photoreceptor. Science, 255(5042):342-344, 1992.

[165] F. T. Hong. Molecular sensors based on the photoelectric effect of bacteriorhodopsin: Origin of differential responsitivity. Materials Science and Engineering, C 5:61-79, 1997.

[166] M. Frydrych, P. Silfsten, S. Parkkinen, J. Parkkinen, and T. Jaaskelainen. Color sensitive retina based on bacteriorhodopsin. BioSystems, 54(3):131-140, 2000. 
[167] D. Haronian and A. Lewis. Elements of a unique bacteriorhodopsin neural network architecture. Applied Optics, 30:597-608, 1991.

[168] D. Haronian and A. Lewis. Microfabricating bacteriorhodopsin films for imaging and computing. Appl. Phys. Lett., 61(18):2237-2239, 1992.

[169] M. Conrad and K.-P. Zauner. Biomaterials for intelligent systems. In M. A. Arbib, editor, The Handbook of Brain Theory and Neural Networks. MIT Press, Cambridge, MA, 1995.

[170] A. B. Druzhko, S. K. Chamorovsky, E. P. Lukashev, A. A. Kononenko, and N. N. Vsevolodov. 4-ketobacteriorhodopsin films as a promising photochromic and electrochromic biological material. BioSystems, 35:129-132, 1995.

[171] N. Vsevolodov. Biomolecular Electronics: An Introduction via Photosensitive Proteins. Birkhäuser, Boston, 1998.

[172] R. R. Birge. Protein-based optical computing and memories. Computer (IEEE), 25:56-67, 1992.

[173] T. Fischer, M. Neebe, T. Juchem, and N. A. Hampp. Biomolecular optical data storage and data encryption. IEEE Transactions on Nanobioscience, 2(1):1-5, 2003.

[174] T. H. Zhang, C .P. Zhang, G. H. Fu, Y. D. Li, L. Q. Gu, G. Y. Zhang, Q. W. Song, B. Parsons, and R. R. Birge. All-optical logic gates using bacteriorhodopsin films. Opt. Eng., 39(2):527-534, 2000.

[175] A. Lewis, Y. Albeck, Z. Lange, J. Benchowski, and G. Weizman. Optical computation with negative light intensity with a plastic bacteriorhodopsin film. Science, 275:1462-1464, 1997.

[176] Munich Innovative Biomaterials GmbH, Marburg, Germany. FringeMaker plus, 2000.

[177] S. K. Scott. Oscillations, waves and chaos in chemical kinetics. Oxford University Press, Oxford, 1994.

[178] H. Malchow. Bistability and spatial pattern formation induced by concentration dependence of reaction rates and diffusion in a nonlinear reaction-electrodiffusion system. Zeitschrift für Physikalische ChemieLeipzig, 271:535-546, 1990.

[179] A. Hjelmfelt, F. W. Schneider, and J. Ross. Pattern recognition in coupled chemical kinetic systems. Science, 260:335-337, 1993.

[180] A. Hjelmfelt and J. Ross. Mass-coupled chemical systems with computational properties. J. Phys. Chem., 97:7988-7992, 1993.

[181] F. R. Pettit. Post-Digital Electronics, chapter 13, pages 161,162,165. Electrical and Electronic Engineering. Ellis Horwood, Chichester, West Sussex, 1982.

[182] N. G. Rambidi. Nondiscrete biomolecular computing. Computer (IEEE), 25:51-54, 1992.

[183] L. Kuhnert. Photochemische Manipulation von chemischen Wellen. Naturwissenschaften, 73:96-97, 1986.

[184] L. Kuhnert, K. I. Agladze, and V. I. Krinsky. Image processing using light-sensitive chemical waves. Nature, 337:244-247, 1989.

[185] L. Hegeüs, H.-D. Försterling, E. Kókai, K. Pelle, G. Taba, M. Wittmann, and Z. Noszticzius. Chemical mechanism of the radical feedback loop in the classical BZ reaction. malonyl bromite and oxalic acid as flow-through intermediates. Phys. Chem. Chem. Phys., 2:4023-4028, 2000.

[186] S. Yan. Mechanistic studies on the Cerium Catalyzed Belousov-Zhabotinsky Reaction. PhD thesis, Fachbereich Chemie, Philipps-Universität Marburg, 2001. Available at http://archiv.ub.uni-marburg.de/diss/z2001/0114/.

[187] A. L. Belmonte, Q. Ouyang, and J.-M. Flesselles. Experimental survey of spiral dynamics in the BelousovZhabotinsky reaction. J. Phys. II France, 7:1425-1468, 1997.

[188] N. G. Rambidi and A. V. Maximychev. Towards a biomolecular computer: information processing capabilities of biomolecular nonlinear dynamic media. BioSystems, 41:195-211, 1997.

[189] K. Agladze, N. Magome, R. Aliev, T. Yamaguchi, and K. Yoshikawa. Finding the optimal path with the aid of chemical wave. Physica D, 106:247-254, 1997.

[190] R. R. Aliev. Oscillating phase dynamics in the Belousov-Zhabotinsky reaction. Implementation to image processing. J. Phys. Chem., 98(15):3999-4002, 1994.

[191] N. G. Rambidi and D. Yakovenchuk. Chemical reaction-diffusion implementation of finding the shortest paths in a labyrinth. Physical Review E, 63:026607-1-6, 2001.

[192] K. Suzuki, T. Yoshinobu, and H. Iwasaki. Induction of chemical waves by mechanical stimulation in elastic Belousov-Zhabotinsky media. Chem. Phys. Lett., 349:437-441, 2001.

[193] K. Suzuki, T. Yoshinobu, and H. Iwasaki. Unidirectional propagation of chemical waves through microgaps between zones with different excitability. J. Phys. Chem. A, 104:6602-6608, 2000.

[194] A. Adamatzky and B. de Lacy Costello. Collision-free path planning in the Belousov-Zhabotinsky medium assisted by a cellular automaton. Naturwissenschaften, 89(10):474-478, 2002.

[195] A. Adamatzky, B. de Lacy Costello, and N. M. Ratcliffe. Experimental reaction-diffusion pre-processor for shape recognition. Physics Letters A, 297:344-352, 2002.

[196] A. Adamatzky and B. de Lacy Costello. Reaction-diffusion path planning in a hybrid chemical and cellularautomaton processor. Chaos, Solitons 83 Fractals, 16(5):727-736, 2003.

[197] O. Steinbock, P. Kettunen, and K. Showalter. Chemical wave logic gates. J. Phys. Chem., 100:18970-18975, 1996.

[198] K. Suzuki, T. Yoshinobu, and H. Iwasaki. Diffusive propagation of chemical waves through a microgap. J. Phys. Chem. A, 104:5154-5159, 2000.

[199] K. Suzuki, T. Yoshinobu, and H. Iwasaki. Anisotropic waves propagating on two-dimensional arrays of Belousov-Zhabotinsky oscillators. Jpn. J. Appl. Phys., 38(Part 2, No. 3B):L345-L348, 1999.

[200] K. Agladze, R. R. Aliev, T. Yamaguchi, and K. Yoshikawa. Chemical diode. J. Phys. Chem., 100:13895$13897,1996$. 
[201] J. Wolff, A. G. Papathanasiou, I. G. Kevrekidis, H. H. Rotermund, and G. Ertl. Spatiotemporal addressing of surface activity. Science, 294(5540):134-137, 2001.

[202] A. G. Hadd, D. E. Raymond, J. W. Halliwell, S. C. Jacobson, and J. M. Ramsey. Microchip device for performing enzyme assays. Anal. Chem., 69:3407-3412, 1997.

[203] M. A. Unger, H.-P. Chou, T. Thorsen, A. Scherer, and S. R. Quake. Monolithic microfabricated valves and pumps by multilayer soft lithography. Science, 288:113-116, 2000.

[204] T. Asai, A. Adamatzky, and Y. Amemiya. Towards reaction-diffusion computing devices based on minoritycarrier transport in semiconductors. Chaos solitons \& fractals, 20(4):863-876, 2004.

[205] J. N. Iraelachvili. Intermolecular and Surface Forces. Academic Press, Boston, 2nd edition, 1992.

[206] D. F. Evans and H. Wennerström. The Colloidal Domain-Where Physics, Chemistry, Biology, and Technology Meet. VCH, Weinheim, 1994.

[207] W. A. Hendrickson, J. L. Smith, and W. E. Royer Jr. Characteristics of protein interfaces. In R. M. Burnett and H. J. Vogel, editors, Biological Organization: Macromolecular Interactions at High Resolution, pages 235-244. Academic Press, Orlando, 1987.

[208] R. A. Atkinson and B. Kieffer. The role of protein motions in molecular recognition: insights from heteronuclear NMR relaxation measurements. Progress in Nuclear Magnetic Resonance Spectroscopy, 44:141-187, 2004.

[209] G. N. Lewis and M. Randall. Thermodynamics, chapter 10 \& 13. McGraw-Hill, New York, 2nd edition, 1961.

[210] M. Conrad. Self-assembly as a mechanism of molecular computing. In Proceedings of the 11th Annual International IEEE-EMBS Conference, pages 1354-1355, Piscataway, NJ, 1989. IEEE.

211] M. Conrad. Quantum mechanics and cellular information processing: The self-assembly paradigm. Biomedica Biochimica Acta, 49:743-755, 1990.

[212] M. Conrad. Molecular computing: The lock-key paradigm. Computer (IEEE), 25(11):11-20, 1992.

[213] M. Conrad and K.-P. Zauner. Conformation-driven computing: a comparison of designs based on DNA, RNA, and protein. Supramolecular Science, 5:787-790, 1998.

[214] F. H. C. Crick. The structure of the hereditary material. In The Physics and Chemistry of Life, pages 118-133. Simon and Schuster, New York, 1955.

[215] J. SantaLucia Jr., H. T. Allawi, and P. A. Seneviratne. Improved nearest-neighbor parameters for predicting DNA duplex stability. Biochemistry, 35:3555-3562, 1996.

[216] J. SantaLucia Jr. A unified view of polymer, dumbbell, and oligonucleotide DNA nearest-neighbor thermodynamics. Proc. Natl. Acad. Sci. USA: Biochemistry, 95:1460-1465, 1998.

[217] J. SantaLucia Jr. and D. Hicks. The thermodynamics of DNA structural motifs. Annu. Rev. Biophys. Biomol. Struct., 33:415-440, 2004.

[218] S. Bommarito, N. Peyret, and J. SantaLucia Jr. Thermodynamic parameter for DNA sequences with dangling ends. Nucleic Acids Research, 28(9):1929-1934, 2000.

[219] M. Zucker. Calculating nucleic acid secondary structure. Current Opinion in Structural Biology, 10:303-310, 2000.

[220] M. Zucker. Mfold web server for nucleic acid folding and hybridization prediction. Nucleic Acids Research, 31(13):3406-3415, 2003.

[221] D. A. Bell. Intelligent Machines, pages 78-79. Blaisdell, New York, 1962.

[222] J. E. Hopcroft, R. Motwani, and J. D. Ullman. Introduction to Automata Theory, Languages, and Computation. Addison Wesley, Boston, 2nd edition, 2001.

[223] A. J. Harwood. Basic DNA and RNA protocols, volume 58 of Methods in Molecular Biology. Humana Press, Totowa, 1996.

[224] M. Linial and N. Linial. On the potential of molecular computing. Science, 268:481, 1995.

[225] A. D. Ellington, M. P. Roberston, K. D. James, and J. C. Cox. Strategies for DNA computing. In H. Rubin and D. H. Wood, editors, DNA Based Computers III, volume 48 of DIMACS Series in Discrete Mathematics and Theoretical Computer Science, pages 173-184, Providence, 1999. American Mathematical Society.

[226] J. C. Cox, D. S. Cohen, and A. D. Ellington. The complexities of DNA computation. Trends in Biotechnology, 17(4):151-154, 1999.

[227] G. Gupta, M. Bansal, and V. Sasisekharan. Polymorphism and conformational flexibility of DNA: right and left duplexes. Int. J. Biol. Macromol., 2:368-380, 1990.

[228] O. Kennard and S. A. Salisburry. DNA structure. In R. A. Meyer, editor, Molecular Biology and Biotechnology, pages 242-247. VCH, Weinheim, 1995.

[229] G. Adam and M. Delbrück. Reduction of dimensionality in biological diffusion processes. In A. Rich and N. Davidson, editors, Structural Chemistry and Molecular Biology, pages 198-215. W. H. Freeman, New York, 1968.

[230] M. Conrad and K.-P. Zauner. DNA as a vehicle for the self-assembly model of computing. BioSystems, 45(1):59-66, 1998.

[231] P. Schuster. A testable genotype-phenotype map: Modeling evolution of RNA molecules. In M. Lässig and A. Valleriani, editors, Biological Evolution and Statistical Physics, pages 56-83. Springer, Berlin, 2002.

[232] P. K. Ponnuswamy and M. M. Gromiha. On the conformational stability of folded proteins. J. theor. Biol., 166:63-74, 1994.

[233] A. R. Davidson and R. T. Sauer. Folded proteins occur frequently in libraries of random amino acid sequences. Proc. Natl. Acad. Sci. USA, 91(6):2146-2150, 1994. 
[234] I. D. Prijambada, T. Yomo, F. Tanaka, T. Kawama, K. Yamamoto, A. Hasegawa, Y. Shima, S. Negoro, and I. Urabe. Solubility of artificial proteins with random sequences. FEBS Lett., 382:21-25, 1996.

[235] A. Yamauchi, T. Yomo, F. Tanaka, I. D. Prijambada, S. Ohhashi, K. Yamamoto, Y. Shima, K. Ogasahara, K. Yutani, M. Kataoka, and I. Urabe. Characterization of soluble artificial proteins with random sequences. FEBS Lett., 421(2):147-151, 1998.

[236] W. J. Netzer and F. U. Hartl. Protein folding in the cytosol: chaperonin-dependent and independent mechanisms. Trends in Biocemical Sciences, 23:68-73, 1998.

[237] R. J. Ellis and F. U. Hartl. Principles of protein folding in the cellular environment. Current Opinion in Structural Biology, 9:102-110, 1999.

[238] M. Karplus. The Levinthal paradox: yesterday and today. Folding $\&$ Design, 2:S69-S75, 1997.

[239] L. C. James and D. S. Tawfik. Conformational diversity and protein evolution - a 60-year-old hypothesis revisited. Trends in Biochemical Sciences, 28(7):361-368, 2003.

[240] S. N. Timasheff. Control of protein stability and reactions by weakly interacting cosolvents: The simplicity of the complicated. Advances in Protein Chemistry, 51:355-432, 1998.

[241] D. K. Eggers and J. S. Valentine. Crowding and hydration effects on protein conformation: A study with sol-gel encapsulated proteins. J. Mol. Biol., 314:911-922, 2001.

[242] M. Conrad. Adaptability: The Significance of Variability from Molecule to Ecosystem, pages 34-36. Pelnum Press, New York, 1983.

[243] M. Conrad. Mutation-absorption model of the enzyme. Bulletin of Mathematical Biology, 41:387-405, 1979.

[244] M. Conrad and M.V. Volkenstein. Replaceability of amino acids and the self-facilitation of evolution. $J$. theor. Biol., 92:293-299, 1981.

[245] M. Conrad. The geometry of evolution. BioSystems, 24:61-81, 1990.

[246] M. Conrad. The brain-machine disanalogy. BioSystems, 22:197-213, 1989.

[247] R. Unger and J. Moult. Finding the lowest free energy conformation of a protein is an NP-hard problem: proof and implications. Bull. Math. Biol., 55:1183-1198, 1993.

[248] N. A. Pierce and E. Winfree. Protein design is NP-hard. Protein Engineering, 15(10):779-782, 2002.

[249] J. D. Honeycutt and D. Thirumalai. Metastability of the folded states of globular proteins. Proc. Natl. Acad. Sci. USA, 87(9):3526-3529, 1990.

[250] R. Merkel. Force spectroscopy on single passive biomolecules and single biomolecular bonds. Physics Reports, 346:343-385, 2001.

[251] G. Bao. Mechanics of biomolecules. Journal of the Mechanics and Physics of Solids, 50:2237-2274, 2002.

[252] G. D. Fasman. Factors responsible for conformational stability. In G. D. Fasman, editor, Poly- $\alpha-A m i n o$ Acids, volume 1 of Biological Macromolecules, pages 499-604. Marcel Dekker, New York, 1967.

[253] S. Kumar, H. J. Wolfson, and R Nussinov. Protein flexibility and electrostatic interactions. IBM J. Res. \& Dev., 45(3/4):499-512, 2001.

[254] F. Parak and E. W. Knapp. A consistent picture of protein dynamics. Proc. Natl. Acad. Sci. USA, 81(22):7088-7092, 1984.

[255] A. Ansari, J. Berendzen, S. F. Bowne, H. Frauenfelder, I. E. T. Iben, T. B. Sauke, E. Shyamsunder, and R. D. Young. Protein states and proteinquakes. Proc. Natl. Acad. Sci. USA, 82(15):5000-5004, 1985.

[256] H. Frauenfelder, F. Park, and R. D. Young. Conformational substates in proteins. Ann. Rev. Biophysics and Biophysical Chem., 17:451-479, 1988.

[257] T. V. Chalikian. Volumetric properties of proteins. Annu. Rev. Biophys. Biomol. Struct., 32:207-235, 2003.

[258] H. Frauenfelder, S. G. Sligar, and P. G. Wolynes. The energy landscapes and motions of proteins. Science, 254:1598-1603, 1991.

[259] H. Frauenfelder and B. McMahon. Dynamics and function of proteins: The search for general concepts. Proc. Natl. Acad. Sci. USA, 95(9):4795-4797, 1998.

[260] L. S. Brown. A local electrostatic change is the cause of the large-scale protein conformational shift in bacteriorhodopsin. Proc. Natl. Acad. Sci. USA, 94(10):5040-5044, 1997.

[261] E. Freire. Statistical thermodynamic linkage between conformational and binding equilibria. Adv.in Prot. Chem., 51:255-279, 1998.

[262] B. Gavish. Molecular dynamics and the transient strain model of enzyme catalysis. In G. R. Welch, editor, The Fluctuating Enzyme, pages 263-339. John Wiley \& Sons, New York, 1986.

[263] E. Fischer. Einfluss der Configuration auf die Wirkung der Enzyme. Berichte der Deutschen Chemischen Gesellschaft (since 1947: Chemische Berichte), 27-3:2985-2993, 1894.

[264] G. Careri and E. Gratton. The statistical time correlation approach to enzyme action: the role of hydration. In G. R. Welch, editor, The Fluctuating Enzyme, pages 227-262. John Wiley \& Sons, New York, 1986.

[265] J. Ninio. Kinetic and probabilistic thinking in accuracy. In T. B. L. Kirkwood, R. F. Rosenberger, and D. J. Galas, editors, Accuracy in Molecular Processes, chapter 10. Chapman and Hall, London, 1986.

[266] J. M. Yon, D. Perahia, and C. Ghélis. Conformational dynamics and enzyme activity. Biochimie, 80:33-42, 1998.

[267] D. E. Koshland. The catalytic and regulatory properties of enzymes. Annu. Rev. Biochem., 37:359-410, 1967.

[268] S. D. Rader and D. A. Agard. Conformational substates in enzyme mechanism: the 120K structure of $\alpha$-lytic protease at 1.5 å resolution. Protein Sci., 6:1375-1386, 1997.

[269] C.-S. Goh, D. Milburn, and M. Gerstein. Conformational changes associated with proteinprotein interactions. Current Opinion in Structural Biology, 14:104-109, 2004. 
[270] N. K. Jerne. Antibody and learning: Selection versus instruction. In G. C. Quarton, T. Melnechuk, and F. O. Schmitt, editors, The Neurosciences, pages 200-205. Rockefeller University Press, New York, 1967.

[271] B. M. Britt. A shifting specificity model for enzyme catatlysis. J. theor. Biol., 164:181-190, 1993.

[272] M. Gerstein, A. M. Lesk, and C. Chothia. Structural mechanisms for domain movements in proteins. Biochemistry, 33(22):6739-6749, 1994.

[273] B. Ma, S. Kumar, C.-J. Tsai, Z. Hu, and R. Nussinov. Transition-state ensemble in enzyme catalysis: Possibility, reality, or necessity? J. theor. Biol., 203:383-397, 2000.

[274] G. G. Hammes. Multiple conformational changes in enzyme catalysis. Biochemistry, 41(26):8221-8228, 2002.

[275] S. Lebreton and B. Gontero. Memory and imprinting in multienzyme complexes. J. Biol. Chem., 274:2087920884, 1999.

[276] L. Edman and R. Rigler. Memory landscapes of single-enzyme molecules. Proc. Natl. Acad. Sci. USA, 97(15):8266-8271, 2000.

[277] H. P. Lu, L. Xun, and X. S. Xie. Single-molecule enzymatic dynamics. Science, 282:1877-1882, 1998.

[278] S. Weiss. Fluorescence spectroscopy of single biomolecules. Science, 283:1676-1683, 1999.

[279] A. Jutila, K. Zhu, S. A. Patkar, J. Vind, A. Svendsen, and P. K. J. Kinnunen. Detergent-induced conformational changes of humicola lanuginosa lipase studied by fluorescence spectroscopy. Biophysical Journal, 78:1634-1642, 2000.

[280] K. E. Prehoda and W. A. Lim. How signaling proteins integrate multiple inputs: a comparison of N-WASP and Cdk2. Current Opinion in Cell Biology, 14:149-154, 2002.

[281] A. Sols. Multimodulation of enzyme activity. Current Topics in Cellular Regulation, 19:77-101, 1981.

[282] H. Frauenfelder, B. H. McMahon, R. H. Austin, K. Chu, and J. T. Groves. The role of structure, energy landscape, dynamics, and allostery in the enzymatic function of myoglobin. Proc. Natl. Acad. Sci. USA, 98(5):2370-2374, 2001.

[283] W. A. Lim. The modular logic of signaling proteins: building allosteric switches from simple binding domains. Current Opinion in Structural Biology, 12:61-68, 2002.

[284] P. Friedrich. Supramolecular Enzyme Organization. Pergamon Press, Oxford, 1984.

[285] D. Bray and T. Duke. Conformational spread: The propagation of allosteric states in large multiprotein complexes. Annu. Rev. Biophys. Biomol. Struct., 33:53-73, 2004.

[286] R. M. Biondi. Phosphoinositide-dependent protein kinase 1, a sensor of protein conformation. Trends in Biocemical Sciences, 29(3):136-142, 2004.

[287] M. Conrad. The seed germination model of enzyme catalysis. BioSystems, 27:223-233, 1992.

[288] M. Sugita. Functional analysis of chemical systems in vivo using a logical circuit equivalent. J. Theo. Biol., 1:415-430, 1961

[289] A. Arkin and J. Ross. Computational functions in biochemical reaction networks. Biophys. J., 67:560-578, 1994.

[290] D. Bray. Protein molecules as computational elements in living cells. Nature, 376:307-312, 1995.

[291] M. H. Capstick, W. P. L. Maranane, and R. Pethig. Biologic computational building blocks. Computer, 25:22-29, 1992.

[292] A. S. Fraenkel. Protein folding, spin glass and computational complexity. In H. Rubin and D. H. Wood, editors, DNA Based Computers III, volume 48 of DIMACS Series in Discrete Mathematics and Theoretical Computer Science, pages 101-121, Providence, 1999. American Mathematical Society.

[293] K.-P. Zauner and M. Conrad. Conformation-driven molecular computing: the optical connection. Optical Memory and Neural Networks, 6(3):157-173, 1997.

[294] K.-P. Zauner and M. Conrad. Conformation-driven computing: simulating the context-conformation-action loop. Supramolecular Science, 5:791-794, 1998.

[295] K.-P. Zauner. Conformation-based Molecular Computing: Simulation and Implementation. PhD thesis, Wayne State University, Detroit, Michigan, 2001.

[296] J. Scheidtmann, P. A. Weiß, and W. F. Maier. Hunting for better catalysts and materials-combinatorial chemistry and high throughput technology. Applied Catalysis A: General, 222:79-89, 2001.

[297] K.-P. Zauner and M. Conrad. Enzymatic pattern processing. Naturwissenschaften, 87(8):360-362, 2000.

[298] K.-P. Zauner and M. Conrad. Enzymatic computing. Biotechnology Progress, 17(3):553-559, 2001.

[299] M. L. Minsky. Some universal elements for finite automata. In C. E. Shannon and J. McCarthy, editors, Automata Studies, number 34 in Annals of Mathematics Studies, pages 117-128. Princeton University Press, Princeton, NJ, 1956.

[300] M. G. Cacace, E. M. Landau, and J. J. Ramsden. The Hofmeister series: salt and solvent effects on interfacial phenomena. Q. Rev. Biophys., 30:241-278, 1997.

[301] A. Dér and J .J. Ramsden. Evidence for loosening of a protein mechanism. Naturwissenschaften, 85:353-355, 1998.

[302] P. C.-P. Wong and A. F. Smith. Assay of serum NAD-dependent malate dehydrogenase using malate as substrate. Clinica Chemica Acta, 72:409-412, 1976.

[303] A. Bracht and A. de P. Campello. Effect of the ionic strength on the kinetic properties of the mitochondrial L-malate dehydrogenase. Experientia, 35:1559-1561, 1979.

[304] E. Kun, R. Z. Eanes, and P. Volfin. Selective modification of mitochondrial malate dehydrogenase activity by changes in ionic strength. Nature, 214:1328-1330, 1967.

[305] A. Ruggia, J. Ll. Gelpí, M. Busquets, M. Cascante, and A. Cortés. Effect of several anions on the activity of mitochondrial malate dehydrogenase from pig heart. J. Mol. Catal. B: Enzymatic, 11:743-755, 2001. 
[306] S. Sivan and N. Lotan. A biochemical logic gate using an enzyme and its inhibitor. 1. The inhibitor as switching element. Biotechnol. Prog., 15:964-970, 1999.

[307] S. Sivan, S. Tuchman, and N. Lotan. A biochemical logic gate using an enzyme and its inhibitor. Part ii: The logic gate. BioSystems, 70:21-33, 2003.

[308] A. S. Deonarine, S. M. Clark, and L. Konermann. Implementation of a multifunctional logic gate based on folding/unfolding transitions of a protein. Future Generation Computer Systems, 19:87-97, 2003.

[309] D. E. Green. Ccxciv. The malic dehydrogenase of animal tissues. Biochem. J., 30:2095-2110, 1936.

[310] C. J. R. Thorne. Properties of mitochondrial malate dehydrogenase. Biochmica et Biophysica Acta, 59:624633, 1962.

[311] D. N. Raval and R. G. Wolfe. Malic dehydrogenase. iii. Kinetic studies of the reaction mechanism by product inhibition. Biochemistry, 1(6):1112-1117, 1962.

[312] D. N. Raval and R. G. Wolfe. Malic dehydrogenase. iv. pH dependence of the kinetic parameters. Biochemistry, 1(6):1118-1123, 1962.

[313] E. Kun. Malate dehydrogenases. In Oxidation and Reduction (Part A), volume 7 of The Enzymes, chapter 7, pages 149-160. Academic Press, New York, 2nd edition, 1963.

[314] C. Cennamo, G. Montecuccoli, and G. König. Inhibition of mitochondrial malate dehydrogenase by citrate. Biochim. Biophys. Acta, 139:514-516, 1967.

[315] E. Silverstein and G. Sulebele. Catalytic mechanism of pig heart mitochondrial malate dehydrogenase studied by kinetics at equilibrium. Biochemistry, 8:2543-2550, 1969.

[316] S. Englard and L. Siegel. Mitochondrial L-malate dehydrogenase of beef heart. In J. M. Lowenstein, editor, Citric Acid Cycle, volume XIII of Methods in Enzymology, pages 99-106. Academic Press, New York, 1969.

[317] R. G. Wolfe and D. N. Raval. The chemical and kinetic properties of pig heart mitochondrial malic dehydrogenase. In The Mechanism and Action of Dehydrogenases. University Press of Kentucky, Lexington, 1970.

[318] L. H. Bernstein, M. B. Grisham, K. D. Cole, and J. Everse. Substrate inhibition of mitochondrial and cytoplasmic malate dehydrogenases. J. Biol. Chem., 253:8697-8701, 1978.

[319] T. R. Mullinax, J. N. Mock, A. J. McEvily, and J. H. Harrison. Regulation of mitochondrial malate dehydrogenase: Evidence for an allosteric citrate-binding site. J. Biol. Chem., 257:13233-13239, 1982.

[320] G. DuVal, H. E. Swaisgood, and H. R. Horton. Some kinetic characteristics of immobilized protomers and native dimers of mitochondrial malate dehydrogenase: An examination of the enzyme mechanism. Biochemistry, 24:2067-2072, 1985.

[321] G. Ll. Gelpí, A. Dordal, J. Montserrat, A. Mazo, and A. Cortés. Kinetic studies of the regulation of mitochondrial malate dehydrogenase by citrate. Biochem. J., 283:289-297, 1992.

[322] S. Shaw, R. Geyer, and G. M. Alter. Dissociation of mitochondrial malate dehydrogenase into active soluble subunits. Biochim. Biophys. Acta: PSME, 1478:248-256, 2000.

[323] J. Adler and W.-W. Tso. "Decision"-making in bacteria: Chemotactic response of Escherichia coli to conflicting stimuli. Science, 184:1292-1294, 1974.

[324] A. Bren and M. Eisenbach. How signals are heard during bacterial chemotaxis: Protein-protein interactions in sensory signal propagation. J. Bacteriol., 182(24):6865-6873, 2000.

[325] M. N. Stojanovic, T. E. Mitchell, and D. Stefanovic. Deoxyribozyme-based logic gates. J. Am. Chem. Soc., 124:3555-3561, 2002.

[326] M. N. Stojanovic and D. Stefanovic. Deoxyribozyme-based half-adder. J. Am. Chem. Soc., 125:6673-6676, 2003.

[327] M. N. Stojanovic and D. Stefanovic. A deoxyribozyme-based molecular automaton. Nature Biotechnology, 21(9):1069-1074, 2003.

[328] R. Heinrich and S. Schuster. The Regulation of Cellular Systems. Chapman \& Hall, New York, 1996.

[329] C. C. Guet, M. B. Elowitz, W. Hsing, and S. Leibler. Combinatorial synthesis of genetic networks. Science, 296:1466-1470, 2002.

[330] G. Biczó. The future of MEBC: panel discussion. BioSystems, 35:233-237, 1995.

[331] Y. Benenson, B. Gil, U. Ben-Dor, R. Adar, and E. Shapiro. An autonomous molecular computer for logical control of gene expression. Nature, 429:423-429, 2004.

[332] M. Hirvensalo. Quantum Computing. Springer, Berlin, 2001.

[333] P. W. Shor. Algorithms for quantum computation: discrete log and factoring. In Proceedings of the 35th Annual IEEE Symposium on Foundations of Computer Science-FOCS, pages 20-22, Piscataway, 1994. IEEE.

[334] S. Lloyd. Universal quantum simulators. Science, 273:1073-1078, 1996.

[335] D. P. Ames. Magnetic resonance. In E. U. Condon and H. Odishaw, editors, Handbook of Physics, chapter 9, pages 8-112-8-127. McGraw-Hill, New York, 2nd edition, 1967.

[336] J. A. Jones. NMR qunatum computing. Progress in Nuclear Magnetic Resonance Spectroscopy, 38:325-360, 2001.

[337] P. O. Boykin, T. Mor, V. Roychowdhury, F. Vatan, , and R. Vrijen. Algorithmic cooling and scalable NMR quantum computers. Proc. Natl. Acad. Sci. USA, 99(6):3388-3393, 2002.

[338] D. Wei, J. Luo, X. Sun, and X. Zeng. Realization of Deutsch-like algorithm using ensemble computing. Physics Letters A, 319:267-272, 2003.

[339] I. L. Chuang, N. Gershenfeld, and M. G. Kubinec. Experimental implementation of fast quantum searching. Phys. Rev. Lett., 80:3408-3411, 1998. 
[340] J. A. Jones, M. Mosca, and R. H. Hansen. Implementation of a quantum search algorithm on a quantum computer. Nature, 393:344-346, 1998.

[341] L. M. K. Vandersypen, M. Steffen, G. Breyta, C. S. Yannoni, M. H. Sherwood, and I. L. Chuang. Experimental realization of Shor's quantum factoring algorithm using nuclear magnetic resonance. Nature, 414:883-887, 2001.

[342] B. E. Kane. A Si-based nuclear-spin quantum computer. Nature, 393:133-138, 1998.

[343] E. Abe, K. M. Itoh, T. D. Ladd, J. R. Goldman, F. Yamaguchi, and Y. Yamamoto. Solid-state silicon NMR quantum computer. Journal of Superconductivity, 16(1):175-178, 2003.

[344] H. Ohta, K. Nomura, H. Hiramatsu, K. Ueda, T. Kamiya, M. Hirano, and H. Hosono. Frontier of transparent oxide semiconductors. Solid-State Electronics, 47:2261-2267, 2003.

[345] K.-P. Zauner and M. Conrad. Conformation-driven molecular computing: The optical connection. Optical Memory and Neural Networks, 6:157-173, 1997.

[346] T. Rueckes, K. Kim, E. Joselevich, G. Y. Tseng, C.-L. Cheung, and C. M. Lieber. Carbon nanotubebased nonvolatile random access memory for molecular computing. Science, 289:94-97, 2000.

[347] T. Aoki, M. Kameyama, and T. Higuchi. Design of a highly parallel set logic network based on a bio-device model. In Proc. 19th Symp. Multiple-Valued Logic, pages 360-367, Washington, DC, 1989. IEEE Computer Society Press.

[348] T. Aoki, M. Kameyama, and T. Higuchi. Interconnection-free biomolecular computing. Computer (IEEE), 25:41-50, 1992.

[349] F. M. Raymo and S. Giordani. Digital communication through intermolecular fluorescence modulation. Organic Letters, 3(12):1833-1836, 2001.

[350] S. D. Varfolomeyev, N. F. Kazanskaya, and N. L. Eremeev. Enzyme activity regulation by an outer physical signal. BioSystems, 39:35-42, 1996.

[351] M. Hiratsuka, T. Aoki, and T. Higuchi. A model of reaction-diffusion cellular automata for massively parallel molecular computing. In 31st IEEE International Symposium on Multiple-Valued Logic, 2001, pages 247-252. IEEE Press, Piscataway, N.J.,, 2001.

[352] C. P. Husband, S. M. Husband, J. S. Daniels, and J. M. Tour. Logic and memory with nanocell circuits. IEEE Transactions on Electron Devices, 50(9):1865-1875, 2003.

[353] R. I. Bahar, J. Mundy, and J. Chen. A probabilistic-based design methodology for nanoscale computation. In International Conference on Computer-Aided Design, San Jose, CA, November 2003, 2003.

[354] G. Pask. An Approach to Cybernetics, chapter 7, pages 100-108. Harper \& Brothers, New York, 1961.

[355] N. C. Seeman. DNA engineering and its application to nanotechnology. Trends in Biotechnology, 17(11):437443, 1999.

[356] N. C. Seeman. DNA in a material world. Nature, pages 427-431, 2003.

[357] E. Winfree. Algorithmic self-assembly of DNA: Theoretical motivations and 2d assembly experiments. $J$. of Biomolecular Structure \& Dynamics, 11(2):263-270, 2000.

[358] M. Cook, P. W. K. Rothemund, and E. Winfree. Self-assembled circuit patterns. In J. Chen and J. H. Reif, editors, DNA Computing: 9th International Workshop on DNA, volume 2943 of Lecture Notes in Computer Science, pages 91-107, Heidelberg, 2004. Springer-Verlag.

[359] E. Winfree and R. Bekbolatov. Proofreading tile sets: Error correction for algorithmic self-assembly. In J. Chen and J. H. Reif, editors, DNA Computing: 9th International Workshop on DNA, volume 2943 of Lecture Notes in Computer Science, pages 126-144, Heidelberg, 2004. Springer-Verlag.

[360] J. J. Storhoff and C. A. Mirkin. Programmed materials synthesis with DNA. Chem. Rev., 99:1849-1862, 1999.

[361] M. R. Diehl, S. N. Yaliraki, R. A. Beckman, M. Barahona, and J. R. Heath. Self-assembled, deterministic carbon nanotube wiring networks. Angew. Chem. Int. Ed., 41(2):353-356, 2002.

[362] D. Liu, S. H. Park, J. H. Reif, and T. H. LaBean. DNA nanotubes self-assembled from triple-crossover tiles as templates for conductive nanowires. Proc. Natl. Acad. Sci. USA, 101(3):717-722, 2004.

[363] S. H. Park, H. Yan, J. H. Reif, T. H. LaBean, and G. Finkelstein. Electronic nanostructures templated on self-assembled DNA scaffolds. Nanotechnology, 15:S525-S527, 2004.

[364] L. Cai and T. Kawai H. Tabata. Self-assembled DNA networks and their electrical conductivity. Applied Physics Letters, 77(19):3105-3106, 2000.

[365] Y. Ito and E. Fukusaki. DNA as a 'nanomaterial'. Journal of Molecular Catalysis B: Enzymatic, 28:155-166, 2004.

[366] P. Ringler and G. E. Schulz. Self-assembly of proteins into designed networks. Science, 302:106-109, 2003.

[367] P. Fairley. Germs that build circuits. IEEE Spectrum, pages 37-41, November 2003.

[368] K. Saitou. Conformational switching in self-assembling mechanical systems. IEEE Transactions on Robotics and Automation, 15(3):510-520, 1999.

[369] M. Sipper, E. Sanchez, D. Mange, M. Tomassini, A. Pérez-Uribe, and A. Stauffer. A phylogenetic, ontogenetic, and epigenetic view of bio-inspired hardware systems. IEEE Transactions on Evolutionary Computation, 1(1):83-97, 1997.

[370] D. Mange, M. Sipper, A. Stauffer, and G. Tempesti. Toward robust integrated circuits: The embryonics approach. Proceedings of the IEEE, 88(4):516-541, 2000.

[371] T. Elliott and N. R. Shadbolt. Developmental robotics: manifesto and application. Phil. Trans. R. Soc. Lond. A, 361:2187-2206, 2003.

[372] A. Schüz. Neuroanatomy in a computational perspective. In M. A. Arbib, editor, The Handbook of Brain Theory and Neural Networks, pages 622-626. MIT Press, Cambridge, MA, 1995. 
[373] J. M. Lehn. Supramolecular chemistry - scope and perspectives; molecules, supermolecules, and molecular devices (Nobel lecture). Angewandte Chemie Int. Ed. Engl., 27:89-112, 1988.

[374] M. Conrad and K.-P. Zauner. Molecular computing with artificial neurons. Commun. KISS, 18(8):78-89, 2000.

[375] M. Karlsson, M. Davidson, R. Karlsson, A. Karlsson, J. Bergenholtz, Z. Konkoli, A. Jesorka, T. Lobovkina, J. Hurtig, M. Voinova, and O. Orwar. Biomimetic nanoscale reactors and networks. Annu. Rev. Phys. Chem., 55:613-649, 2004.

[376] S. A. Benner. Act natural-synthetic biology. Nature, 421:118, 2003.

[377] W. J. Blake and F. J. Isaacs. Synthetic biology evolves. Trends in Biotechnology, 22(7):321-324, 2004.

[378] J. Ziegler, P. Dittrich, and W. Banzhaf. Towards a metabolic robot control system. In M. Holcombe and R. Paton, editors, Information Processing in Cells and Tissues, pages 305-317. Plenum Press, New York, 1998.

[379] J. Ziegler and W. Banzhaf. Evolving control metabolism for a robot. Artificial Life, 7(2):171-190, 2001.

[380] A. Adamatzky, B. de Lacy Costello, C. Melluish, and N. Ratcliffe. Experimental implementation of mobile robot taxis with onboard Belousov-Zhabotinsky chemical medium. Materials Science 83 Engineering $C$, 24(4):541-548, 2004.

[381] J. Millman and H. Taub. Pulse and Digital Circuits, pages 564-567. McGraw-Hill, New York, 1956.

[382] J. Bardeen and W. H. Brattain. The transistor, a semi-conductor triode. Phys. Rev., 74:230-231 (L), 1948.

[383] R. K. Richards. Electronic Digital Systems, page 38. Wiley, New York, 1966.

[384] S. Toulmin and J. Goodfield. The Architecture of Matter. Harper \& Row, New York, 1962. 